\title{
Super real closed rings
}

\author{
by
}

\author{
Marcus Tressl (Passau)
}

\begin{abstract}
A super real closed ring is a commutative ring equipped with the operation of all continuous functions $\mathbb{R}^{n} \rightarrow \mathbb{R}$. Examples are rings of continuous functions and super real fields attached to $z$-prime ideals in the sense of Dales and Woodin. We prove that super real closed rings which are fields are an elementary class of real closed fields which carry all o-minimal expansions of the real field in a natural way. The main part of the paper develops the commutative algebra of super real closed rings, by showing that many constructions of lattice ordered rings can be performed inside super real closed rings; the most important are: residue rings, complete and classical quotients, convex hulls, valuations, Prüfer hulls and real closures over proconstructible subsets. We also give a counterexample to the conjecture that the first order theory of (pure) rings of continuous functions is the theory of real closed rings, which says in addition that a semi-local model is a product of fields.
\end{abstract}

\section{Contents}

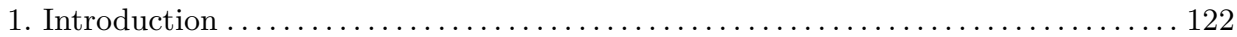

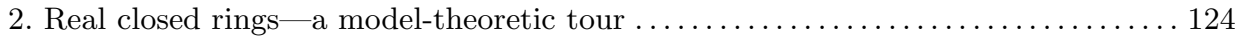

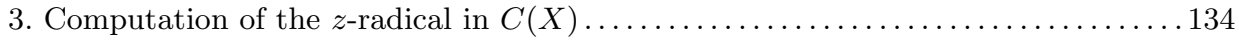

4. A sentence in the language of rings separating continuous semi-algebraic from

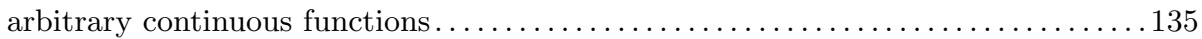

5. Super real closed rings: Definition and basic properties $\ldots \ldots \ldots \ldots \ldots \ldots \ldots \ldots 141$

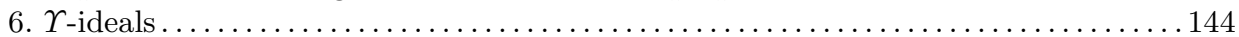

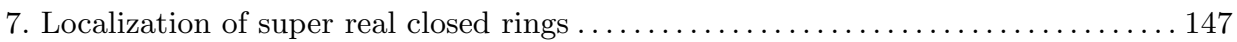

8. Application: o-minimal structures on super real closed fields . . . . . . . . . . . 152

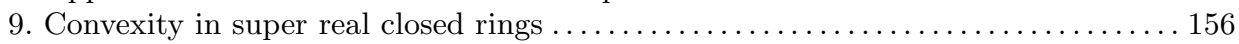

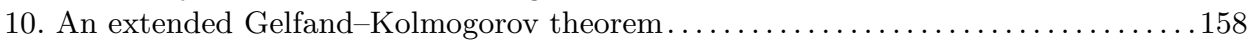

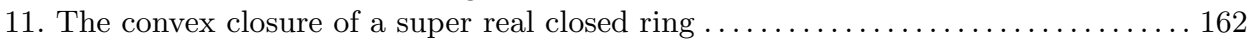

12. Real closures over proconstructible subsets of $\Upsilon$-Spec $A \ldots \ldots \ldots \ldots \ldots \ldots \ldots \ldots \ldots$

13. The complete ring of quotients of a super real closed $\operatorname{ring} \ldots \ldots \ldots \ldots \ldots \ldots \ldots \ldots \ldots$

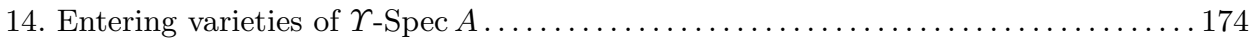

2000 Mathematics Subject Classification: Primary 03C60; Secondary 46E25, 54C05, $03 \mathrm{E} 15$.

Key words and phrases: real closed rings, super real fields, rings of continuous functions, model theory, convexity, spectra.

Partially supported by the DFG project no. SCHW 287/17-1 and by the European RTNetwork RAAG, contract no. HPRN-CT-2001-00271. 
1. Introduction. A super real field (cf. [Da-Wo]) is the quotient field of the ring $C(X)$ of continuous, real-valued functions on a topological space $X$ divided by a prime ideal $\mathfrak{p}$ of $C(X)$. The ideal $\mathfrak{p}$ is called a prime $z$-ideal if $f \in \mathfrak{p}$ whenever $f$ vanishes on a zero set of some $g \in \mathfrak{p}$. Take a prime $z$-ideal $\mathfrak{p}$ of $C(X)$ and let $K:=\mathrm{qf}(C(X) / \mathfrak{p})$ be the corresponding super real field.

The initial motivation for this paper was to prove that for every ominimal expansion $\mathcal{R}$ of the real field (cf. [vdD]) in a first order language $\mathscr{L}$ extending the language for ordered unital rings, there is a natural expansion $\mathfrak{M}$ of the field $K$ to an $\mathscr{L}$-structure such that $\mathfrak{M}$ is an elementary extension of $\mathcal{R}$.

This is indeed true (cf. (8.5)(ii)) and it turns out that a commutative algebra lies behind this fact, namely the algebra of super real closed rings: A super real closed ring is a commutative unital ring $A$ together with maps $F_{A}: A^{n} \rightarrow A$ for each continuous function $F: \mathbb{R}^{n} \rightarrow \mathbb{R}(n \in \mathbb{N})$ such that the composition rules for the functions $F_{A}$ are the same as for the original functions $F$, i.e.

$$
F_{A} \circ\left(G_{1, A}, \ldots, G_{n, A}\right)=\left(F \circ\left(G_{1}, \ldots, G_{n}\right)\right)_{A} .
$$

Moreover, addition, multiplication and the identity of $\mathbb{R}$ have to be interpreted as addition, multiplication and the identity of $A$.

Obviously, super real closed rings are precisely the models of a first order theory $T_{\Upsilon}$ in a language $\mathscr{L}_{\Upsilon}$ extending the language of rings, which has function symbols for each continuous $F: \mathbb{R}^{n} \rightarrow \mathbb{R}$. Examples are:

(i) Every ring of real-valued continuous functions has a natural expansion to a super real closed ring.

(ii) Every super real field at a $z$-prime ideal as described above has a natural expansion to a super real closed field (cf. (8.5)(i)).

The answer to the motivating question is given in Theorem (8.5)(ii), which says that every super real closed ring which is a field carries all ominimal expansions of $\mathbb{R}$ (in the sense described above). Hence $T_{\Upsilon}+$ "fields" is a theory of real closed fields, which act as resplendent structures inside the class of all o-minimal expansions of fields, stemming from $\mathbb{R}$.

A real closed ring is a commutative unital ring $A$ together with maps $F_{A}: A^{n} \rightarrow A$ for each semi-algebraic continuous function $F: \mathbb{R}^{n} \rightarrow \mathbb{R}$ $(n \in \mathbb{N})$, defined over $\mathbb{Q}$, such that $(*)$ holds for these functions and addition, multiplication and the identity of $\mathbb{R}$ are interpreted as addition, multiplication and the identity of $A$, respectively. This notion has been introduced by N. Schwartz in [Schw1]. The original definition is purely ring-theoretic, i.e. there is no mention of the $F_{A}$. The formulation above is in part implicitly contained in [Schw-Ma, Section 12]. We introduce real closed rings in this way and we show that the functions $F_{A}$ are definable in the pure real closed ring (by an existential Horn formula, cf. Theorem (2.12)). 
Real closed rings provide a very flexible category of rings in which the algebra of the rings $C(X)$ can be studied. One purpose of this article is to show that most of this flexibility is also present in the category of super real closed rings. The axioms of super real closed rings, which imply that the class of models of $T_{\Upsilon}$ together with $\mathscr{L}_{\Upsilon}$-morphisms is a variety in the sense of universal algebra, promise this: we get many basic constructions like direct and inverse limits, fibre sums and fibre products, free objects, inside this variety for free (cf. end of Section 5).

In Sections 6, 7 and 9-13, classical manipulations of commutative rings, or better, of lattice ordered rings, are established inside the category of super real closed rings. The motor which makes the theory work is a Nullstellensatz for rings of continuous functions, which expresses an algebraic relation between continuous functions that have the same zero set (cf. Section 3, which reviews parts of $[\operatorname{Tr} 1])$.

By classical manipulations we initially mean "developing a theory of ideals and localizations" for super real closed rings (see Sections 6 and 7). The appropriate notion of an ideal in a super real closed ring is of course "the kernel of a ring homomorphism into a super real closed ring, which respects all the $F_{A}$ ". These ideals are called $\Upsilon$-ideals; the name is explained in Section 6.

The set $\Upsilon$-Spec $A$ of all $\Upsilon$-ideals of a super real closed ring which are prime forms a spectral space (cf. [Hoc]), more precisely, a spectral subspace of $\operatorname{Spec} A$. As in the case of real closed rings, the analysis of the ring is intimately connected with this topological space, as well as with the full spectrum of $A$. In Section 14, a principal geometric difference between the spectrum of super real closed rings and that of real closed rings of semialgebraic functions is proved. We explain this intuitively as follows (the details can be found in Section 14): If $A$ is a super real closed ring and $V \subseteq$ Spec $A$ is the set of prime ideals containing a given element $a \in A$, then the only way to enter this variety from the outside is by walking through generic points of $V$. But in the semi-algebraic context, say when $V$ corresponds to the closed unit disc in $\mathbb{R}^{n}, V$ can only be entered by passing through the boundary of $V$, and this boundary is not generic in $V$.

It is not clear if the difference above can be described in a purely ringtheoretic manner and the question arises if "real closed rings" is the first order theory of super real closed rings, when viewed as pure rings. In Section 4 we show that the ring of continuous semi-algebraic functions $\mathbb{R} \rightarrow \mathbb{R}$ satisfies a sentence in the language of rings which is not satisfied by any ring of continuous functions $C(X)$. The sentence "almost" says that the pure field $\mathbb{R}$ is o-minimal. I do not know whether a super real closed ring is a model of that sentence. The result in Section 4 says that either "real closed rings" is not the intersection of the theory of super real closed rings with the sentences in the language of rings, or super real closed rings are 
not the first order description of the class of rings of continuous functions in the language $\mathscr{L}_{\Upsilon}$.

2. Real closed rings - a model-theoretic tour. Let $R$ be a real closed field and let $\Lambda \subseteq R$ be a subring. Recall that a $\Lambda$-semi-algebraic subset of $R^{n}$ is a boolean combination of sets of the form $\{P \geq 0\}:=\{x \in$ $\left.R^{n} \mid P(x) \geq 0\right\}$, where $P$ is a polynomial over $\Lambda$ in $n$ variables. A map $f: R^{n} \rightarrow R^{m}$ is called $\Lambda$-semi-algebraic if the graph of $f$ is a $\Lambda$-semialgebraic subset of $R^{n} \times R^{m}$. If $\Lambda=R$, then we say semi-algebraic instead of " $\Lambda$-semi-algebraic".

(2.1) Definition. Let $R$ be a real closed field. For $n, r \in \mathbb{N}_{0}$, let

$\mathcal{C}_{R^{n}}^{r}:=\left\{f: R^{n} \rightarrow R \mid f\right.$ is semi-algebraic and $r$-times continuously differentiable\}.

Moreover we set

$$
\mathcal{C}_{R^{n}}^{-1}:=\left\{f: R^{n} \rightarrow R \mid f \text { is semi-algebraic }\right\} .
$$

Given $r \in \mathbb{N}_{0} \cup\{-1\}$, an $R$-real closed ring of class $C^{r}$ is a commutative unitary ring $A$ together with a collection of functions $\left(f_{A}\right)_{n \in \mathbb{N}}, f \in \mathcal{C}_{R^{n}}^{r}$, where $f_{A}: A^{n} \rightarrow A$, with the following properties:

1. If $f$ is constantly 0 or constantly 1 , then so is $f_{A}$; if $f: R \rightarrow R$ is the identity, then so is $f_{A}: A \rightarrow A$; if $f: R^{2} \rightarrow R$ is addition or multiplication, then so is $f_{A}: A^{2} \rightarrow A$.

2. If $k \in \mathbb{N}$ and $f_{i} \in \mathcal{C}_{R^{k}}^{r}(1 \leq i \leq n)$, then

$$
\left[f \circ\left(f_{1}, \ldots, f_{n}\right)\right]_{A}=f_{A} \circ\left(f_{1, A}, \ldots, f_{n, A}\right) .
$$

If $r=0$, then we say $R$-real closed instead of " $R$-real closed of class $C^{r}$ ". If $R$ is the field $\mathbb{R}_{\text {alg }}$ of real algebraic numbers, then we say real closed of class $C^{r}$ instead of " $R$-real closed of class $C^{r}$ ". If $r=0$ and $R=\mathbb{R}_{\text {alg }}$, then we simply say real closed.

Note. We do not require that $1 \neq 0$. Hence the null ring is also considered to be $R$-real closed of class $C^{r}$.

(2.2) Observation. Every real closed ring is reduced.

Proof. Let $a \in A$ be such that $a^{3}=0$. Let $f: \mathbb{R}_{\text {alg }} \rightarrow \mathbb{R}_{\text {alg }}$ be defined by $f(x)=\sqrt[3]{x}$. Then $f$ is continuous and semi-algebraic with $f \circ g=\mathrm{id}_{\mathbb{R}_{\mathrm{alg}}}$, where $g(x)=x^{3}$. By definition we have $f_{A} \circ g_{A}(b)=b$ and $g_{A}(b)=b^{3}$ for all $b \in A$. Hence $a=f_{A} \circ g_{A}(a)=f_{A}(0)=0$.

If $r>0$, then a real closed ring of class $C^{r}$ is not necessarily reduced (cf. (2.14)). Many properties of real closed rings below come for free also for $r>0$ provided the ring is reduced. 
In this paper we are mainly interested in the case $r=0$, which is covered by the reduced case as we have just observed. The general (nonreduced) case $r>0$ will be the subject of another paper ([Tr2]). However, in order not to repeat arguments we include the case $r>0$ in this article whenever it is not substantially different from the case $r=0$.

The pure rings underlying real closed rings in our sense are precisely the real closed rings in the sense of Schwartz [Schw1]. This is contained in [Schw-Ma, Section 12], and at first sight our definition seems to be only of theoretical use. The opposite is the case. We shall prove that

(a) If $A$ is a reduced, real closed ring of class $C^{r}$, then there is exactly one collection $\left(f_{A}\right)$ of functions as in Definition (2.1) and each function $f_{A}$ is definable in $A$ in the language of rings by an existential Horn formula (cf. [Ho, 9.4]). This is proved in (2.12). (Warning: in general there is no quantifier free formula in the language of rings which defines the graph of $f_{A}$ : in particular, a formula which defines the graph of $f$ will not define the graph of $f_{A}$ in general.)

(b) Every ring homomorphism between reduced $R$-real closed rings of class $C^{r}$ respects the unique (by (a)) additional structures. This is proved in (2.16).

Items (a) and (b) say that the category of reduced real closed rings of class $C^{r}$ (and ring homomorphisms respecting the new symbols) is a full subcategory of the category of rings (and ring homomorphisms). Our definition easily allows generalizations of well known functorial constructions from commutative algebra to the category of real closed rings of class $C^{r}$ which are reduced, e.g. direct limits and fibre sums. The reason is that the category of real closed rings of class $C^{r}$ (reduced or not) together with ring homomorphisms respecting the new symbols is - by definition - a variety in the sense of universal algebra; item (b) above implies that many functorial constructions inside the category of rings can be performed inside the category of real closed rings of class $C^{r}$.

We occasionally work with the natural first order language of real closed rings of class $C^{r}$. Throughout we shall work with the language $\mathscr{L}:=$ $\{+,-, \cdot, 0,1\}$ of unital rings.

(2.3) Definition. Let $\mathscr{L}\left(\mathrm{RCR}^{r}\right)$ be the language $\mathscr{L}$ together with an $n$-ary function symbol $\underline{f}$ for every semi-algebraic, function $f: \mathbb{R}_{\text {alg }}^{n} \rightarrow \mathbb{R}_{\text {alg }}$ of class $C^{r}$. Let $\mathrm{RCR}^{r}$ be the $\mathscr{L}\left(\mathrm{RCR}^{r}\right)$-theory which has the following axioms:

1. The axioms of a commutative unital ring in the language $\{+,-, \cdot, 0,1\}$.

2. $\forall x y \pm(x, y)=x+y \wedge$ : $(x, y)=x \cdot y \wedge \underline{\operatorname{id}}(x)=x \wedge$ 二 $(x)=-x \wedge$ $\underline{1}(x)=1$. Hence the symbols from the language of rings have the 
same meaning as the corresponding symbols when reintroduced in $\mathscr{L}\left(\mathrm{RCR}^{r}\right)$ as symbols naming semi-algebraic $C^{r}$-functions.

3. All the sentences

$$
\begin{aligned}
& \forall \bar{x} \underline{f}\left(\underline{f_{1}}(\bar{x}), \ldots, \underline{f_{n}}(\bar{x})\right)=\underline{f \circ\left(f_{1}, \ldots, f_{n}\right)}(\bar{x}), \\
& \text { with } f \in C_{\mathbb{R}_{\text {alg }}^{n}}^{r} \text { and } f_{1}, \ldots, f_{n} \in C_{\mathbb{R}_{\text {alg }}^{r}}^{r} .
\end{aligned}
$$

Clearly the models of $\mathrm{RCR}^{r}$ are exactly the real closed rings of class $C^{r}$, where the symbols $f$ are interpreted as $f_{A}$. Observe again that the null ring is a model of $\mathrm{RCR}^{r}$.

(2.4) Remark. The partial order on a real closed ring $A$ of class $C^{r}$ is given by $x \leq y \Leftrightarrow y-x=n(y-x)$, where $n \in C_{\mathbb{R}_{\text {alg }}}$ is defined by $n(t):=|t|^{r+1}$. Hence $x \leq y$ is definable by a positive atomic $\mathscr{L}\left(\mathrm{RCR}^{r}\right)$ formula and we may view it as an abbreviation for this formula.

(2.5) Lemma. Let $R$ be a real closed field and let $X \subseteq R^{n}$ be $\Lambda$-semialgebraic for a subring $\Lambda$ of $R$. Then $X$ is defined by a formula $\exists \bar{u} P(\bar{x}, \bar{u})$ $=0$ for some polynomial $P(\bar{x}, \bar{u}) \in \Lambda[\bar{x}, \bar{u}]$. Applying this to the complement of $X$ shows that $X$ is also defined by a formula $\forall \bar{u} P(\bar{x}, \bar{u}) \neq 0$ for some polynomial $P(\bar{x}, \bar{u}) \in \Lambda[\bar{x}, \bar{u}]$.

Proof. By quantifier elimination, $X$ is defined by $\bigvee_{i}\left(P_{i}(\bar{x})=0 \wedge\right.$ $\left.\bigwedge_{j} Q_{i j}(\bar{x})>0\right)$ for some polynomials $P_{i}, Q_{i j} \in \Lambda[\bar{x}]$. Then $X$ is defined by $\exists \bar{u} P(\bar{x}, \bar{u})=0$ with

$$
P(\bar{x}, \bar{u}):=\prod_{i}\left(P_{i}(\bar{x})^{2}+\sum_{j}\left(Q_{i j}(\bar{x}) \cdot u_{i j}^{2}-1\right)^{2}\right) .
$$

(2.6) Proposition. Let $r \in \mathbb{N}_{0}$ and let $R$ be a real closed field (or more generally an o-minimal structure, $c f$. [vdD]). If $\varepsilon \in R, \varepsilon>0$ and $f:(-\varepsilon, \varepsilon) \rightarrow R$ is continuous, definable in $R$ with $f(0)=0$, which is of class $C^{r}$ on $(-\varepsilon, \varepsilon) \backslash\{0\}$, then $f(x) \cdot x^{r}$ is of class $C^{r}$ on $(-\varepsilon, \varepsilon)$.

Proof. This is folklore; we sketch the proof. Firstly, o-minimality implies that for every $R$-definable $g: R \rightarrow R$ there is some $r$ such that $g:(r, \infty) \rightarrow R$ is differentiable and $\lim _{x \rightarrow \infty} x \cdot g^{\prime}(x)=0$ if $\lim _{x \rightarrow \infty} g(x)=0$. Using this property one shows the assertion by induction on $r$.

(2.7) Lemma And Definition. Let $r \in \mathbb{N}_{0}$. Let $X \subseteq R^{n}$ be $\Lambda$-semialgebraic for a subring $\Lambda$ of $R$. Then $X$ is closed if and only if $X$ is defined by a formula $\exists \bar{u} P(\bar{x}, \bar{u})=0$ for some polynomial $P(\bar{x}, \bar{u}) \in \Lambda[\bar{x}, \bar{u}]$ such that there is a 1-semi-algebraic map $s: R^{\bar{x}} \rightarrow R^{\bar{u}}$ of class $C^{r}$ with

$$
R \models \forall \bar{x} \in X P(\bar{x}, s(\bar{x}))=0 .
$$

Here and below we write $R^{\bar{v}}$ instead of $R^{\text {length }(\bar{v})}$ if $\bar{v}$ is a tuple of variables. We call such a polynomial a $C^{r}$-representation of $X$ and the map s a $C^{r}$ section of $P$. 
Proof. If $X$ is defined by a formula as indicated, then $X=f^{-1}(0)$ for the map $f: R^{\bar{x}} \rightarrow R, f(\bar{x}):=P(\bar{x}, s(\bar{x}))$. Since $f$ is continuous, $X$ is closed.

Conversely, suppose $X$ is closed. By the finiteness theorem (cf. [BCR, Thm. 2.7.2]), $X$ is defined by a formula

$$
\bigvee_{i} \bigwedge_{j} P_{i j}(\bar{x}) \geq 0
$$

for some polynomials $P_{i j}(\bar{x}) \in \Lambda[\bar{x}]$. Take

$$
P(\bar{x}, \bar{u}):=\prod_{i} \sum_{j}\left(P_{i j}(\bar{x})^{2 r+1}-u_{i j}^{2}\right)^{2} .
$$

Clearly $X$ is defined by $\exists \bar{u} P(\bar{x}, \bar{u})=0$. Let $s_{i j}: R^{\bar{x}} \rightarrow R$ be defined by

$$
s_{i j}(\bar{x}):={\sqrt{\left|P_{i j}(\bar{x})\right|}}^{2 r+1} \text {. }
$$

Let $s:=\left(s_{i j}\right)$. Then $s$ is a $\Lambda$-semi-algebraic map $R^{\bar{x}} \rightarrow R^{\bar{u}}$ with

$$
R \models \forall \bar{x} \in X P(\bar{x}, s(\bar{x}))=0 .
$$

It is of class $C^{r}$, since the function $\sqrt{|x|}^{2 r+1}=\left(\operatorname{sign}\left(x^{r}\right) \cdot \sqrt{|x|}\right) \cdot x^{r}$ is of class $C^{r}$ by $(2.6)$.

We extend the notation to $r=-1$ and arbitrary, definable $X \subseteq R^{n}$ : Every polynomial $P(\bar{x}, \bar{u}) \in \Lambda[\bar{x}, \bar{u}]$ such that $X$ is defined by $\exists \bar{u} P(\bar{x}, \bar{u})=0$ is called a $C^{-1}$-representation of $X$. Observe that in this case, there is a $C^{-1}$-map $s: R^{\bar{x}} \rightarrow R^{\bar{u}}$ with $R=\forall \bar{x} \in X P(\bar{x}, s(\bar{x}))=0$, since the theory $\mathrm{RCF}$ of real closed fields has definable Skolem functions.

(2.8) Definition. Let $X$ be a set, let $R$ be a real closed field and let $\Lambda$ be a subring of $R$. If $S$ is a set of functions $X \rightarrow R$ and $r \in \mathbb{N}_{0} \cup\{-1\}$ we define

$$
\begin{aligned}
\operatorname{csa}_{\Lambda}^{r}(S):=\left\{f \circ\left(a_{1}, \ldots, a_{n}\right) \mid n \in \mathbb{N}, a_{1}, \ldots, a_{n} \in S\right. \text { and } \\
\\
\left.\quad f \in \mathcal{C}_{R^{n}}^{r}, \Lambda \text {-semi-algebraic }\right\} .
\end{aligned}
$$

The notation is correct, since by quantifier elimination for real closed fields, the ring $\operatorname{csa}_{\Lambda}^{r}(S)$ does not depend on $R$, but only on the ordered ring $\Lambda$.

If $r=-1$ then we also write $\operatorname{sa}_{\Lambda}(S)$ instead of $\operatorname{csa}_{\Lambda}^{-1}(S)$. If $\Lambda=\mathbb{Z}$ then we suppress the subscript $\Lambda$.

Observe that $\Lambda$, viewed as a subring of constant functions of $R^{X}$, is contained in $\operatorname{csa}_{\Lambda}^{r}(S)$. Moreover it is obvious that $\operatorname{csa}_{\Lambda}^{r}(S)$ is a ring of functions $X \rightarrow R$ and $\operatorname{csa}_{\Lambda}^{r}\left(\operatorname{csa}_{\Lambda}^{r}(S)\right)=\operatorname{csa}_{\Lambda}^{r}(S)$.

(2.9) Lemma. Let $A$ be a ring of functions $X \rightarrow R$, let $\Lambda$ be a subring of $R$, and assume $A=\operatorname{csa}_{\Lambda}^{r}(A)$. Let $Z \subseteq R^{\bar{z}}$ be closed and $\Lambda$-semi-algebraic 
with $C^{r}$-representation $P(\bar{z}, \bar{u}) \in \Lambda[\bar{z}, \bar{u}]$. Then

$$
Z_{A}:=\left\{\bar{a} \in A^{\bar{z}} \mid \text { for all } x \in X, \bar{a}(x) \in Z\right\}
$$

is definable in $A$ with parameters from $\Lambda$ by $\exists \bar{u} P(\bar{z}, \bar{u})=0$.

Proof. If $A=\exists \bar{u} P(\bar{a}, \bar{u})=0$, then clearly $\bar{a} \in Z_{A}$, since $Z$ is defined by $\exists \bar{u} P(\bar{z}, \bar{u})=0$. Conversely if $\bar{a} \in Z_{A}$, then take a $\Lambda$-semi-algebraic map $s: R^{\bar{z}} \rightarrow R^{\bar{u}}$ of class $C^{r}$ with

$$
R \models \forall \bar{z} \in Z P(\bar{z}, s(\bar{z}))=0
$$

and let $\bar{c}:=s(\bar{a}) \in A^{\bar{u}}$. Then $A \models P(\bar{a}, \bar{c})=0$, since for each $x \in X$ we have $\bar{a}(x) \in Z$, hence $R \models P(\bar{a}(x), s(\bar{a}(x)))=0$.

(2.10) Lemma. Let $A$ be a ring of functions $X \rightarrow R$ from a set $X$ to a real closed field $R$ and let $\Lambda$ be a subring of $R$ and $A$. Let $f: R^{n} \rightarrow R$ be semi-algebraic, let $P(\bar{v}, y, \bar{u}) \in \Lambda[\bar{v}, y, \bar{u}]$ be such that the graph of $f$ is defined by the formula $\varphi(\bar{v}, y)=\exists \bar{u} P(\bar{v}, y, \bar{u})=0$, and let $s: R^{\bar{v}} \rightarrow R^{\bar{u}}$ be a semi-algebraic map with

$$
R \models \forall \bar{v} P(\bar{v}, f(\bar{v}), s(\bar{v}))=0 .
$$

(i) If $\bar{a} \in A^{n}$ and $b \in A$ with $A=\varphi(\bar{a}, b)$, then $b=f \circ \bar{a}$.

(ii) If $A=\forall \bar{v} \exists y \varphi(\bar{v}, y)$ then for every $\bar{a} \in A^{n}$ we have $f \circ \bar{a} \in A$ and $\varphi(\bar{v}, y)$ defines the graph of the map $f_{A}: A^{n} \rightarrow A, f_{A}(\bar{a}):=f \circ \bar{a}$.

(iii) If $f \circ \bar{a}, s \circ \bar{a} \in A^{\bar{u}}$ for all $\bar{a} \in A^{n}$, then $A=\forall \bar{v} \exists y \varphi(\bar{v}, y)$.

Proof. (i) Take $(\bar{a}, b) \in A^{n} \times A$ with $A=\varphi(\bar{a}, b)$. We have to show that $b=f \circ \bar{a}$. Pick $x \in X$. Since $A=\varphi(\bar{a}, b)$, there is some $\bar{c} \in A^{\bar{u}}$ with $P(\bar{a}, b, \bar{c})=0$ in $A$. Thus $P(\bar{a}(x), b(x), \bar{c}(x))=0$ and $R=\exists \bar{u} P(\bar{a}(x), b(x), \bar{u})$ $=0$. This means $b(x)=f(\bar{a}(x))$.

(ii) is an immediate consequence of (i).

(iii) Let $\bar{a} \in A^{n}$ and take $b:=f \circ \bar{a}$. Then $b \in A$ and $A=\exists \bar{u} P(\bar{a}, b, \bar{u})=0$ as $\bar{c}:=s \circ \bar{a} \in A^{\bar{u}}$ satisfies $P(\bar{a}, b, \bar{c})=0$.

(2.11) Proposition. Let $r \in \mathbb{N}_{0} \cup\{-1\}$ and let $A$ be a ring of functions $X \rightarrow R$ from a set $X$ to a real closed field $R$. Let $\Lambda$ be a subring of $R$ and $A$. Then $A=\operatorname{csa}_{\Lambda}^{r}(A)$ if and only if for every $n \in \mathbb{N}_{0}$ and every $\Lambda$ semi-algebraic map $f: R^{n} \rightarrow R$ of class $C^{r}$, there is a $C^{r}$-representation $P(\bar{v}, y, \bar{u}) \in K[\bar{v}, y, \bar{u}]$ of the graph of $f$ with

$$
A \models \forall \bar{v} \exists y \bar{u} P(\bar{v}, y, \bar{u})=0 .
$$

If this is the case, then for every $\Lambda$-semi-algebraic map $f: R^{n} \rightarrow R$ of class $C^{r}$, and every $C^{r}$-representation $P(\bar{v}, y, \bar{u}) \in \Lambda[\bar{v}, y, \bar{u}]$ of the graph of $f$, the formula $\exists \bar{u} P(\bar{v}, y, \bar{u})=0$ defines the graph of the map

$$
f_{A}: A^{n} \rightarrow A, \quad f_{A}(\bar{a}):=f \circ \bar{a} .
$$

Proof. By (2.10) and (2.7). 
(2.12) Theorem. Let $r \in \mathbb{N}_{0} \cup\{-1\}$, let $R$ be a real closed field and let $\Lambda$ be a subring of $R$ such that $R$ is the real closure of the ordered ring $\Lambda$. Let $A$ be a commutative unital $\Lambda$-algebra.

(i) Suppose $A$ can be expanded to an $R$-real closed ring $\mathcal{A}$ of class $C^{r}$. Then

(a) For each $\Lambda$-semi-algebraic map $f: R^{n} \rightarrow R$ of class $C^{r}$, every $C^{r}$-representation $P(\bar{v}, y, \bar{u}) \in \Lambda[\bar{v}, y, \bar{u}]$ of the graph of $f$ and all $C^{r}$-sections s of $P$ we have $\mathcal{A}=\forall \bar{v} P\left(\bar{v}, f_{\mathcal{A}}(\bar{v}), s_{\mathcal{A}}\left(\bar{v}, f_{\mathcal{A}}(\bar{v})\right)\right)$ $=0$. In particular $\mathcal{A}=\forall \bar{v} \exists y \bar{u} P(\bar{v}, y, \bar{u})=0$.

(b) $A / \operatorname{Nil}(A)$ is real reduced, i.e. $a_{1}^{2}+\cdots+a_{n}^{2} \in \operatorname{Nil}(A)$ implies $a_{1}, \ldots, a_{n} \in \operatorname{Nil}(A)$ for all $a_{1}, \ldots, a_{n} \in A$. Here $\operatorname{Nil}(A)$ denotes the nilradical of $A$.

(c) If $A$ is reduced, then for each $\Lambda$-semi-algebraic map $f: R^{n} \rightarrow R$ of class $C^{r}$ and all $C^{r}$-representations $P(\bar{v}, y, \bar{u}) \in \Lambda[\bar{v}, y, \bar{u}]$ of the graph of $f$, the function $f_{\mathcal{A}}: A^{n} \rightarrow A$ is defined by $\exists \bar{u} P(\bar{v}, y, \bar{u})=0$. In particular $\mathcal{A}$ is the unique expansion of $A$ to an $R$-real closed ring $\mathcal{A}$ of class $C^{r}$.

(ii) Suppose $A$ is reduced and for all $n \in \mathbb{N}$, each $\Lambda$-semi-algebraic map $f: R^{n} \rightarrow R$ of class $C^{r}$ and every $C^{r}$-representation $P(\bar{v}, y, \bar{u}) \in$ $\Lambda[\bar{v}, y, \bar{u}]$ of the graph of $f$ we have

$$
A \models \forall \bar{v} \exists y \bar{u} P(\bar{v}, y, \bar{u})=0 .
$$

Then $A$ can be expanded to an $R$-real closed ring of class $C^{r}$.

Proof. (i)(a) If $f: R^{n} \rightarrow R$ is $\Lambda$-semi-algebraic of class $C^{r}$ and $P(\bar{v}, y, \bar{u})$ $\in \Lambda[\bar{v}, y, \bar{u}]$ is a $C^{r}$-representation of the graph of $f$ with $C^{r}$-section $s$ : $R^{\bar{v}} \times R \rightarrow R^{\bar{u}}$, then $R=\forall \bar{v} P(\bar{v}, f(\bar{v}), s(\bar{v}, f(\bar{v})))=0$. Hence the axioms of

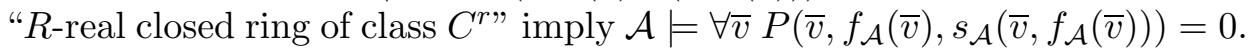

(i) (b) Take $a_{1}, \ldots, a_{n} \in A$ such that $a_{1}^{2}+\cdots+a_{n}^{2}$ is nilpotent. We must show that each $a_{i}$ is nilpotent. By the division property for semi-algebraic $C^{r}$-functions there is some $p \in \mathbb{N}$ and a $\mathbb{Z}$-semi-algebraic $C^{r}$-function $d$ : $R^{n} \rightarrow R$ such that $x_{i}^{p}=\left(x_{1}^{2}+\cdots+x_{n}^{2}\right) \cdot d\left(x_{1}, \ldots, x_{n}\right)$ on $\mathbb{R}_{\text {alg }}^{n}$ (alternatively one can directly show by induction on $r$ that $x_{i}^{p}$ with $p=2^{r+1}+1$ is divisible by $x_{1}^{2}+\cdots+x_{n}^{2}$ in $\left.C_{R}^{r}\right)$. Again the axioms of " $R$-real closed ring of class $C^{r}$ " imply $a_{i}^{p}=\left(a_{1}^{2}+\cdots+a_{n}^{2}\right) \cdot d_{A}\left(a_{1}, \ldots, a_{n}\right)$, which is nilpotent. This shows that $A / \operatorname{Nil}(A)$ is real reduced.

(i)(c) If $A$ is reduced, then by (i)(b), $A$ is real reduced, hence isomorphic to a ring of functions $X \rightarrow R^{\prime}$ from a set $X$ to a real closed field $R^{\prime} \supseteq R$. We may assume that $A$ is this ring of functions $X \rightarrow R^{\prime}$. But then (i)(c) holds by (2.11) and (i)(a).

(ii) It is enough to show that $A$ is real reduced; then (ii) follows from (2.11) as in the proof of (i)(c). Let $a_{1}, \ldots, a_{n} \in A$ with $a_{1}^{2}+\cdots+a_{n}^{2}=0$. 
We must show that $a_{i}=0$ for all $i$. Take $p \in \mathbb{N}$ and a $\mathbb{Z}$-semi-algebraic $C^{r}$-function $d: R^{n} \rightarrow R$ such that $x_{i}^{p}=\left(x_{1}^{2}+\cdots+x_{n}^{2}\right) \cdot d\left(x_{1}, \ldots, x_{n}\right)$ on $\mathbb{R}_{\mathrm{alg}}^{n}$. Then the polynomial $P(\bar{v}, y):=\left(v_{1}^{2}+\cdots+v_{n}\right)^{2} \cdot y-v_{i}^{p} \in \Lambda[\bar{v}, y]$ is a $C^{r}$-representation of the graph of $d$. By assumption (ii) we have

$$
A \models \forall \bar{v} \exists y P(\bar{v}, y)=0 .
$$

Hence by specializing $v_{i}$ to $a_{i}$, there is some $b \in A$ such that $a_{i}^{p}=\left(a_{1}^{2}+\cdots\right.$ $\left.+a_{n}\right)^{2} \cdot b=0$. Since $A$ is assumed to be reduced, we get $a_{i}=0$ as desired.

(2.13) Corollary. Let $r \in \mathbb{N}_{0} \cup\{-1\}$, let $R$ be a real closed field, and let $\Lambda$ be a subring of $R$ such that $R$ is the real closure of the ordered ring $\Lambda$. Then for every reduced, commutative unital $\Lambda$-algebra $A$ the following are equivalent:

(i) $A$ can be expanded to an $R$-real closed ring of class $C^{r}$.

(ii) For all $n \in \mathbb{N}$, each $\Lambda$-semi-algebraic map $f: R^{n} \rightarrow R$ of class $C^{r}$ and every $C^{r}$-representation $P(\bar{v}, y, \bar{u}) \in \Lambda[\bar{v}, y, \bar{u}]$ of the graph of $f$ we have

$$
A \models \forall \bar{v} \exists y \bar{u} P(\bar{v}, y, \bar{u})=0 .
$$

If this is the case, then there is a unique expansion $\mathcal{A}$ of $A$ to an $R$-real closed ring of class $C^{r}$ and the functions $f_{\mathcal{A}}: A^{n} \rightarrow A$ are defined by $\exists \bar{u} P(\bar{v}, y, \bar{u})=0$ whenever $f \in C_{R^{n}}^{r}$ and $P(\bar{v}, y, \bar{u}) \in \Lambda[\bar{v}, y, \bar{u}]$ is a $C^{r}$ representation of the graph of $f$.

Proof. Directly from (2.12).

By (2.2) every real closed ring is reduced (and therefore real reduced by $(2.12)(\mathrm{i})(\mathrm{b}))$. For $r>0$ this is not true any more:

(2.14) Example. Here is an example of an $R$-real closed ring of class $C^{1}$ which is not reduced.

Let $R$ be a real closed field. Recall that by o-minimality, every semialgebraic function $R \rightarrow R$ which is differentiable at a point is of class $C^{1}$ in a neighbourhood of that point. Let $B$ be the ring of all $f \in C_{R}^{0}$ which are differentiable in $R \backslash\{0\}$. For $q \in \mathbb{Q}, q>0$, let $I_{q}$ be the principal ideal of $B$ generated by $x \cdot\left(x^{2}\right)^{q}$, hence

$$
I_{q}:=x \cdot\left(x^{2}\right)^{q} \cdot B .
$$

Claim 1. For $q_{1}, q_{2} \in \mathbb{Q}$ with $0<q_{2}<q_{1}$ we have $I_{q_{1}} \subseteq I_{q_{2}} \subseteq C_{R}^{1}$, in particular

$$
I:=\bigcup_{q>0} I_{q}
$$

is an ideal of $C_{R}^{1}$.

Proof. By (2.6) we have $I_{q} \subseteq C_{R}^{1}$ for all $q>0$. 
If $0<q_{2}<q_{1}$, then $x \cdot\left(x^{2}\right)^{q_{1}}=x \cdot\left(x^{2}\right)^{q_{2}} \cdot\left(x^{2}\right)^{q_{1}-q_{2}}$. As $x^{q_{1}-q_{2}} \in B$ this shows that $x \cdot\left(x^{2}\right)^{q_{1}} \in I_{q_{2}} \cdot B=I_{q_{2}}$, thus $I_{q_{1}} \subseteq I_{q_{2}}$ and Claim 1 is proved.

Our example now is $A:=C_{R}^{1} / I$, which is not a reduced ring since $x^{3} \in I$ and $x \notin I$. We have to define the operation of definable functions $R^{n} \rightarrow R$ of class $C^{1}$ on $A$ :

Take $F \in C_{R^{n}}^{1}$ and $f_{1}, \ldots, f_{n} \in C_{R}^{1}$. We define

$$
F_{A}\left(f_{1} \bmod I, \ldots, f_{n} \bmod I\right):=F\left(f_{1}, \ldots, f_{n}\right) \bmod I
$$

and we need only show that $F_{A}$ is well defined. Take $g_{1}, \ldots, g_{n} \in C_{R}^{1}$ with $h_{i}:=f_{i}-g_{i} \in I(1 \leq i \leq n)$. We must show that $F(\bar{f})-F(\bar{g})$ is divisible by some $x \cdot\left(x^{2}\right)^{q}$ in $B$. By replacing $F$ with $F\left(x_{1}+f_{1}(0), \ldots, x_{n}+f_{n}(0)\right)$ and $f_{i}$ with $f_{i}-f_{i}(0)$ we may assume that $f_{i}(0)=0$ for all $i$.

Since $F$ is differentiable at 0 we have

$$
F(\bar{x})=F(0)+\sum_{i=1}^{n} \frac{\partial F}{\partial x_{i}}(0) \cdot x_{i}+|\bar{x}| \cdot \psi(\bar{x}),
$$

where $\psi: R^{n} \rightarrow R$ is a function with $\lim _{\bar{x} \rightarrow 0} \psi(\bar{x})=0$. Since $F$ is semi-algebraic and differentiable on $R^{n}, \psi$ is semi-algebraic, differentiable on $R^{n} \backslash\{0\}$ and continuous on $R^{n}$.

Claim 2. For every semi-algebraic curve $s: R \rightarrow R^{n}$ of class $C^{1}$ with $s(0)=0$ the function $|s(x)| \cdot \psi(s(x))$ is in $I$.

Proof. Since $\psi(s(x))$ is semi-algebraic and continuous on $R$ with $\psi(s(0))$ $=0$, there are $q \in \mathbb{Q}, q>0$, such that $a:=\lim _{x \rightarrow 0} \psi(s(x)) /\left(x^{2}\right)^{q}$ exists in $R$. Since $s$ is differentiable at 0 with $s(0)=0$, also $b:=\lim _{x \rightarrow 0}|s(x)| / x$ exists in $R$. Hence

$$
t(x):=\frac{|s(x)| \cdot \psi(s(x))}{x \cdot\left(x^{2}\right)^{q}} \quad(x \neq 0), \quad t(0)=a \cdot b,
$$

defines a continuous semi-algebraic function. By $(*)$ and since $s$ is differentiable, $|s(x)| \cdot \psi(s(x))$ is differentiable on $R$. Hence $t$ is differentiable at all points $x \neq 0$, in other words $t \in B$. Thus $|s(x)| \cdot \psi(s(x))=x \cdot\left(x^{2}\right)^{q} \cdot t(x)$ $\in I$, which shows Claim 2 .

Now we can show that $F(\bar{f}(x))-F(\bar{g}(x)) \in I$. We have

$$
F(\bar{f}(x))-F(\bar{g}(x))=\sum_{i=1}^{n} \frac{\partial F}{\partial x_{i}}(0) \cdot h_{i}+|\bar{f}(x)| \cdot \psi(\bar{f}(x))-|\bar{g}(x)| \cdot \psi(\bar{g}(x)) .
$$

By Claim 2, $|\bar{f}(x)| \cdot \psi(\bar{f}(x))-|\bar{g}(x)| \cdot \psi(\bar{g}(x)) \in I$ and as $h_{i} \in I$, also $\sum_{i=1}^{n} \frac{\partial F}{\partial x_{i}}(0) \cdot h_{i} \in I$. This finishes the example.

In the example above we have: 
(i) $C_{R}^{1} / I \cong R+x \cdot R$ and after identification

$$
F_{A}\left(a_{1}+x b_{1}, \ldots, a_{n}+x b_{n}\right)=F(\bar{a})+\sum_{i=1}^{n} \frac{\partial F}{\partial x_{i}}(\bar{a}) \cdot b_{i} .
$$

This follows from Claim 2 in the example above.

(ii) The assertion in (i)(c) of (2.12) does not hold for the real closed ring $A=C / I$ of class $C^{1}$, i.e. there is a representation $P(\bar{v}, y, \bar{u})$ of a definable $C^{1}$-function such that the function $f_{\mathcal{A}}: A^{n} \rightarrow A$ is not defined by $\exists \bar{u} P(\bar{v}, y, \bar{u})=0$. Take $f(x)=x \cdot \sqrt[3]{x}$ and $P(v, y, \bar{u})=$ $y^{3}-v^{4}$. In $A$, the formula $y^{3}-v^{4}=0$ does not define the graph of a function since the equation $y^{3}=0=x^{4} \bmod I$ has infinitely many solutions in $A$ : all elements $r \cdot x \bmod I, r \in R$.

We state other consequences of (2.12):

(2.15) Corollary. Let $r \in \mathbb{N}$ and let $R$ be a real closed field. Let $A$ be an $R$-real closed ring of class $C^{r}$ and let $I \subseteq A$ be a radical ideal. Then there is a unique expansion of $A / I$ to an $R$-real closed ring of class $C^{r}$ and the residue map $A \rightarrow A / I$ is an $\mathscr{L}_{R}^{r}$-homomorphism. This applies in particular to $I=\operatorname{Nil}(A)$.

Proof. By (2.12)(i)(a), the pure ring $A$ satisfies all sentences $\forall \bar{v} \exists y \bar{u}$ $P(\bar{v}, y, \bar{u})=0$, where $P$ runs through the $C^{r}$-representations of $\Lambda$-semialgebraic $C^{r}$-functions. Since these sentences are positive, and the residue map $A \rightarrow A / I$ is surjective, also $A / I$ satisfies all these sentences (without any assumption on the ideal $I$ ). Since $A / I$ is reduced, there is a unique expansion of $A / I$ to an $R$-real closed ring of class $C^{r}$ (cf. (2.12)(ii)) and it remains to show that the residue map respects the $f_{A}$. But this follows from $(2.12)(\mathrm{i})(\mathrm{a})$ and $(\mathrm{i})(\mathrm{c})$.

(2.16) Corollary. Let $\varphi: A \rightarrow B$ be a ring homomorphism between real closed rings of class $C^{r}$. If $B$ is reduced, then $\varphi$ is an $\mathscr{L}\left(\mathrm{RCR}^{r}\right)$-homomorphism.

Proof. We must show that $\varphi\left(f_{A}\left(a_{1}, \ldots, a_{n}\right)\right)=f_{B}\left(\varphi\left(a_{1}\right), \ldots, \varphi\left(a_{n}\right)\right)$ for all $a_{1}, \ldots, a_{n} \in A$ and each semi-algebraic map $f: \mathbb{R}_{\text {alg }}^{n} \rightarrow \mathbb{R}_{\text {alg }}$ of class $C^{r}$. Let $P(\bar{v}, y, \bar{u}) \in \mathbb{Z}[\bar{v}, y, \bar{u}]$ be a $C^{r}$-representation of the graph of $f$. Let $a_{0}:=f_{A}\left(a_{1}, \ldots, a_{n}\right)$. By $(2.12)(\mathrm{i})(\mathrm{a}), A \models \exists \bar{u} P\left(a_{1}, \ldots, a_{n}, a_{0}, \bar{u}\right)=0$. Since $P$ is a polynomial with coefficients in $\mathbb{Z}$ it follows that $B=\exists \bar{u}$ $P\left(\varphi\left(a_{1}\right), \ldots, \varphi\left(a_{n}\right), \varphi\left(a_{0}\right), \bar{u}\right)=0$. Since $B$ is reduced we have $f_{B}\left(\varphi\left(a_{1}\right), \ldots\right.$, $\left.\varphi\left(a_{n}\right)\right)=\varphi\left(a_{0}\right)$ by $(2.12)(\mathrm{i})(\mathrm{c})$.

(2.17) Theorem And Definition. Every commutative ring $A$ has a (reduced) real $C^{r}$-closure $(B, f)$, i.e. $B$ is a (reduced) real closed ring of class $C^{r}, f$ is a ring homomorphism $f: A \rightarrow B$ and for every ring homo- 
morphism $g: A \rightarrow B^{\prime}$ with values in a (reduced) real closed ring of class $C^{r}$ there is a unique $\mathscr{L}\left(\mathrm{RCR}^{r}\right)$-homomorphism $h: B \rightarrow B^{\prime}$ such that $g=h \circ f$.

We write $\varrho^{r}(A)$ for the real $C^{r}$-closure of $A$.

Proof. The theory $\mathrm{RCR}^{r}$ as well as the theory red- $\mathrm{RCR}^{r}$ of reduced real closed rings of class $C^{r}$ in the language $\mathscr{L}\left(\mathrm{RCR}^{r}\right)$ is strict universal Horn, i.e. axiomatized by sentences of the form

$$
\forall \bar{x} \varphi_{1} \wedge \cdots \wedge \varphi_{d} \rightarrow \psi
$$

where $\varphi_{i}$ and $\psi$ are positive atomic (cf. [Ho, 9.1]). This follows from the shape of the axioms of $\mathrm{RCR}^{r}$ and since reducedness is expressed by the strict universal formula $\forall x x^{2}=0 \rightarrow x=0$. Let $T$ be $\mathrm{RCR}^{r}$ or red-RCR ${ }^{r}$. By the general theory of presentations (cf. [Ho, 9.2]), we know that for every set of positive atomic $\mathscr{L}\left(\mathrm{RCR}^{r}\right)(C)$-sentences, where $C$ is a set of new constants, the term algebra (cf. [Ho, 1.2]) of $T \cup \Phi$ is again a model of $T$.

We take $C=A$ and $\Phi$ to be the set of positive atomic $\mathscr{L}(A)$-sentences, valid in $A$ (recall that $\mathscr{L}$ is the language of rings). Then the term algebra of red-RCR ${ }^{r} \cup \Phi$ can be viewed as a ring homomorphism $f: A \rightarrow B$, where $B$ is a model of red-RCR ${ }^{r}$. By the correspondence between models of diagrams and morphisms (cf. [Ho, 1.4]), this gives the assertion.

(2.18) Corollary. Let $A$ be a ring and let $B:=\varrho^{r}(A) / \operatorname{Nil}\left(\varrho^{r}(A)\right)$.

(i) The composition $\bar{f}$ of the natural map $f: A \rightarrow \varrho^{r}(A)$ with the residue map $\varrho^{r}(A) \rightarrow B$ is the reduced real $C^{r}$-closure of $A$.

(ii) If $A$ is real reduced, then $\bar{f}$ is injective.

(iii) The reduced real $C^{r}$-closure can be entirely defined inside the category of rings: $B$ is the commutative ring which can be expanded to a reduced, real closed ring of class $C^{r}, \bar{f}$ is a ring homomorphism $A \rightarrow B$ and for every ring homomorphism $g: A \rightarrow B^{\prime}$ into a ring $B^{\prime}$ which can also be expanded to a reduced real closed ring of class $C^{r}$, there is a unique ring homomorphism $h: B \rightarrow B^{\prime}$ with $g=h \circ \bar{f}$.

(iv) If $A=\varrho^{r}(A)$ and $I$ is a radical ideal of $A$, then also $A / I$ is a real closed ring of class $C^{r}$.

Proof. (i) By (2.16), the residue map $\varrho^{r}(A) \rightarrow \varrho^{r}(A) / \operatorname{Nil}\left(\varrho^{r}(A)\right)$ is an $\mathscr{L}\left(\mathrm{RCR}^{r}\right)$-homomorphism. Hence (i) follows from the functorial definition of both closures in (2.17).

(ii) If $A$ is real reduced, then $A$ can be embedded into a product of real closed fields. This product is a real closed ring and since the embedding factors through $\bar{f}, \bar{f}$ must be injective.

Item (iii) is straightforward from (i) and (2.16), (2.12), and item (iv) is straightforward from (ii) and (2.16), (2.12). 
3. Computation of the $z$-radical in $C(X)$. First we recall standard notions for rings of continuous functions (cf. [Gil-Jer]). Let $X$ be a Tikhonov space, i.e. a completely regular Hausdorff space. Let $C(X)$ be the ring of continuous functions $X \rightarrow \mathbb{R}$. Then $C(X)$ is a subring and a sublattice of the distributive lattice $\mathbb{R}^{X}$. Recall that for every topological space $Y$ there is a Tikhonov space $X$ such that $C(X)$ is isomorphic to $C(Y)$ (cf. [Gil-Jer, 3.9]). Moreover, Tikhonov spaces are precisely the subspaces of compact spaces.

A zero set of $X$ is a set of the form $\{f=0\}:=\{x \in X \mid f(x)=0\}$, with $f \in C(X)$. A cozero set of $X$ is a set of the form $\{f \neq 0\}:=\{x \in X \mid$ $f(x) \neq 0\}$ with $f \in C(X)$. Observe that any set of the form $\{f \geq 0\}$ with $f \in C(X)$ is a zero set, since $\{f \geq 0\}=\{f \wedge 0=0\}$.

An ideal $\mathfrak{a}$ of $C(X)$ is a $z$-ideal if $f \in \mathfrak{a}$ whenever $f$ vanishes on a zero set of a function from $\mathfrak{a}$. The $z$-radical $\sqrt[z]{\mathfrak{a}}$ of an arbitrary ideal $\mathfrak{a}$ of $C(X)$ is the smallest $z$-ideal of $C(X)$ containing $\mathfrak{a}$.

Let

$$
\Upsilon:=\left\{s: \mathbb{R} \rightarrow \mathbb{R} \mid s \text { is continuous and } s^{-1}(0)=\{0\}\right\} .
$$

(3.1) Definition. An ideal $\mathfrak{a}$ of $C(X)$ is called $\Upsilon$-radical if $s \circ f \in \mathfrak{a}$ for all $f \in \mathfrak{a}$ and all $s \in \Upsilon$. Since the intersection of $\Upsilon$-radical ideals is obviously again $\Upsilon$-radical, we may define the $\Upsilon$-radical $\sqrt[\Upsilon]{\mathfrak{a}}$ of an ideal $\mathfrak{a}$ of $C(X)$ as the smallest $\Upsilon$-radical ideal of $C(X)$ containing $\mathfrak{a}$.

Clearly $\Upsilon$-radical ideals are radical and every $z$-radical ideal of $C(X)$ is $\Upsilon$-radical.

(3.2) Definition. A subset $\Upsilon_{0}$ of $\Upsilon$ is called a set of generalized root functions if for all $s \in \Upsilon$, there are $s_{0} \in \Upsilon_{0}$ and $\varepsilon \in \mathbb{R}$ with $\varepsilon>0$ and $|s| \leq\left|s_{0}\right|$ on $(0, \varepsilon)$.

A subset $\Upsilon_{0}$ of $\Upsilon$ is called a set of generalized power functions if for all $s \in \Upsilon$, there are $s_{0} \in \Upsilon_{0}$ and $\varepsilon \in \mathbb{R}$ with $\varepsilon>0$ and $\left|s_{0}\right| \leq|s|$ on $(0, \varepsilon)$.

(3.3) Proposition $([\operatorname{Tr} 1,(5.12)])$. Let $\mathfrak{a}$ be an ideal of $C(X)$. Then

(i) For every set $\Upsilon_{0} \subseteq \Upsilon$ of generalized root functions we have

$$
\sqrt[r]{\mathfrak{a}}=\left\{g \cdot(s \circ f) \mid g \in C(X), f \in \mathfrak{a}, f \geq 0, s \in \Upsilon_{0}\right\} .
$$

(ii) For every set $\Upsilon_{0} \subseteq \Upsilon$ of generalized power functions we have

$$
\sqrt[\Upsilon]{\mathfrak{a}}=\left\{f \in C(X)|s \circ| f \mid \in \mathfrak{a} \text { for some } s \in \Upsilon_{0}\right\} .
$$

(3.4) Proposition ([Tr1, (5.7)]). If $\mathfrak{a}$ is an ideal of $C(X)$, then there is a largest $\Upsilon$-radical $\mathfrak{a}^{\Upsilon}$ contained in $\mathfrak{a}$ and for every set $\Upsilon_{0} \subseteq \Upsilon$ of generalized root functions we have

$$
\mathfrak{a}^{\Upsilon}=\left\{F \in \mathfrak{a} \mid s_{0} \circ F, s_{0} \circ(-F) \in \mathfrak{a} \text { for all } s_{0} \in \Upsilon_{0}\right\} .
$$


The foregoing propositions imply functoriality of the $\Upsilon$-radical:

(3.5) Proposition ([Tr1, (5.13)]). If $\tau: X \rightarrow Y$ is a continuous map between Tikhonov spaces $X, Y$ and $\varphi: C(Y) \rightarrow C(X)$ is the corresponding ring homomorphism $($ so $\varphi(g)=g \circ \tau)$ then for every ideal $\mathfrak{a}$ of $C(X)$ we have

$$
\sqrt[\Upsilon]{\varphi^{-1}(\mathfrak{a})}=\varphi^{-1}(\sqrt[\Upsilon]{\mathfrak{a}}) \quad \text { and } \quad \varphi^{-1}(\mathfrak{a})^{\Upsilon}=\varphi^{-1}\left(\mathfrak{a}^{\Upsilon}\right) .
$$

(3.6) Definition. A Tikhonov space $X$ has computable $z$-radicals if $\sqrt[r]{\mathfrak{a}}=\sqrt[z]{\mathfrak{a}}$ for all ideals $\mathfrak{a}$ of $C(X)$.

(3.7) Theorem ([Tr1, (8.9) and (8.15)]). A cozero set $X$ of a compact space has computable $z$-radicals. A subset $X$ of $\mathbb{R}^{n}$ has computable $z$-radicals if and only if $X$ is locally closed.

\section{A sentence in the language of rings separating continuous} semi-algebraic from arbitrary continuous functions. The first order theory of the pure ring $C(X)$ is undecidable if $X$ is a nondiscrete metric space. This has been shown by Cherlin in [Che]. This section contributes (in a negative way) to the problem what the theory $T$ of the class of all $C(X)$ in the language of rings is. It was conjectured that this theory is "almost equal" to the theory of real closed rings (more precisely, that $T$ is $\mathrm{RCR}^{0}$ plus the set of sentences which asserts that a semi-local ring which is a model of $T$ is a product of fields). We disprove this conjecture by showing that rings of semi-algebraic functions are not a model of $T$. Explicitly:

Let $M$ be an o-minimal structure expanding a real closed field (cf. [vdD]). Let $C_{M}$ be the ring of $M$-definable, continuous maps $M \rightarrow M$. Then $C_{M}$ is not a model of the theory of all $C(X)$, more precisely we construct a first order sentence in the language of rings which holds in each $C_{M}$ and in no $C(X)$.

This will also disprove the conjecture that the theory of the class of all rings $C(X)$ together with all the quotients $C(X) / \mathfrak{p}(\mathfrak{p} \in \operatorname{Spec} C(X))$ is the theory of real closed rings (cf. [PS]); otherwise $C_{M}$ can be elementarily embedded into an ultraproduct of rings of the form $C(X)$ or $C(X) / \mathfrak{p}$-since $C_{M}$ is not a domain, we may assume that it is actually an ultraproduct of rings of the form $C(X)$; but then the above mentioned formula must hold in at least one $C(X)$.

We now construct this sentence. First we construct several auxiliary sentences and formulas in the language of rings. The notation $C_{M}$ below always means the ring of $M$-definable, continuous maps $M \rightarrow M$ for an o-minimal expansion of a real closed field. $X$ always denotes a Tikhonov space. 
1. Let $x \prec y$ be an abbreviation for the formula

$$
\forall u \exists v w 1=w \cdot y+v \cdot(1+u \cdot x) .
$$

Then for every $\operatorname{ring} A$ and all $a, b \in A$ we have $a \prec b \Leftrightarrow a$ is in the Jacobson radical $\sqrt[\max ]{b \cdot A}:=\bigcap_{\mathfrak{m} \in(\operatorname{Spec} A)^{\max }, b \in \mathfrak{m}} \mathfrak{m}$ (this is well known by basic commutative algebra).

If $a, b \in A$ then we write $a \succ b$ if $a \succ b$ and $a \prec b$.

2. Let $A$ be a ring of functions from a set $S$ to a field $K$. We say that $S$ is weakly represented in $A$ if for all $f, g \in A$ we have $f \prec g \Leftrightarrow\{g=0\} \subseteq$ $\{f=0\}$. In this case $f \in A$ is a unit in $A$ if and only if $f$ has no zeros in $S$.

Indeed, if $f$ is a unit in $A$, then clearly $f$ does not have zeros in $S$. Conversely, if $f$ is a nonunit in $A$, then $1 \nprec f$, hence $\{f=0\} \not \subseteq\{1=0\}=\emptyset$.

For example $M$ is weakly represented in $C_{M}$ and $X$ is weakly represented in $C(X)$.

3. Let $A$ be ring of functions from a set $S$ to a field $K$. We say that $S$ is represented in $A$ if $S$ is weakly represented in $A$ and if for all $s, t \in S$ there is some $f \in A$ with $f(s)=0$ and $f(t) \neq 0$.

For example $M$ is represented in $C_{M}$ and $X$ is represented in $C(X)$.

From now on, $A$ denotes a real closed ring of functions $S \rightarrow K$ for some set $S$ and some real closed field $K$.

4. Let $\operatorname{pt}(x)$ be the formula

$$
\operatorname{pt}(x):=\forall u \quad u x \neq 1 \wedge[\forall y(\forall u u y \neq 1) \wedge x \prec y \rightarrow y \prec x] .
$$

Then for each $a \in A$ we have $A=\operatorname{pt}(a) \Leftrightarrow a$ is a maximal element in the set of nonunits of $A$, with respect to the relation $\prec$. We write $\operatorname{pt}(A)$ for the realizations of $\operatorname{pt}(x)$ in $A$ and call these realizations point functions.

For each $f \in A$ we have $A=\operatorname{pt}(f)$ if and only if the Jacobson radical of $f$ is a maximal ideal, i.e. there is a unique maximal ideal of $A$ containing $f$.

We define

$$
\operatorname{Max}_{\mathrm{pt}}(A):=\{\mathfrak{m} \in \operatorname{Max} A \mid \exists f \in A: \mathfrak{m}=\sqrt[\max ]{f \cdot A}\}
$$

and we call the elements of $\operatorname{Max}_{\mathrm{pt}}(A)$ the points of $A$. Hence there is a 1-1 correspondence between $\operatorname{Max}_{\mathrm{pt}}(A)$ and the realizations of $\mathrm{pt}(x)$ in $A$ modulo the relation $\prec$ (which is equal to $\prec$ on $\operatorname{pt}(A)$ ). For short, $\operatorname{Max}_{\mathrm{pt}}(A)=$ $\operatorname{pt}(A) / \succ$.

5. If $S$ is represented by $A$, then for each $f \in A$ we have

$$
A=\operatorname{pt}(f) \Leftrightarrow f \text { has exactly one zero in } S \text {. }
$$

Indeed, clearly $A=\operatorname{pt}(f)$ if $f$ has exactly one zero in $S$. Conversely, suppose $A=\operatorname{pt}(f)$. Then $f$ has zeros in $S$. Suppose $s, t$ are two such zeros. Since $S$ is represented in $A$ there is $g \in A$ with $g(s)=0 \neq g(t)$. But then $f \prec g^{2}+f^{2}$ and $g^{2}+f^{2} \nprec f$, a contradiction to $A=\operatorname{pt}(f)$. 
6. Let DENSE and DENSE ${ }^{\text {con }}$ be the following sentences in the language of rings:

$$
\begin{aligned}
\mathrm{DENSE} & :=\forall x x \neq 0 \rightarrow \exists z \operatorname{pt}(z) \wedge x \nprec z, \\
\operatorname{DENSE}^{\mathrm{con}} & :=\forall x, y \neg x \prec y \rightarrow \exists z \operatorname{pt}(z) \wedge y \prec z \wedge \neg x \prec z .
\end{aligned}
$$

Then $A=\operatorname{DENSE} \Leftrightarrow \operatorname{Max}_{\mathrm{pt}}(A)$ is dense in Spec $A$ and $A=\operatorname{DENSE}^{\text {con }} \Leftrightarrow$ $\operatorname{Max}_{\mathrm{pt}}(A)$ is dense in $(\operatorname{Spec} A)^{\mathrm{con}}$, i.e. in $\operatorname{Spec} A$ equipped with the constructible topology.

7. Let

$$
P:=\{s \in S \mid\{s\} \text { is a zero set of } A\} .
$$

If $S$ is represented in $A$, then

$$
A=\mathrm{DENSE} \Leftrightarrow P \text { is dense in } S \text { with respect to }
$$

the cozero-topology induced by $A$,

i.e. with respect to the topology on $S$ which has the sets $\{f \neq 0\}(f \in A)$ as a basis of open sets. In particular, if $A=C(X)$, then $A \models$ DENSE $\Leftrightarrow$ $P$ is dense in $X$.

Moreover

$$
A \models \mathrm{DENSE}^{\mathrm{con}} \Leftrightarrow P \text { is dense in } S \text { with respect to }
$$

the constructible topology induced by $A$,

i.e. with respect to the topology on $S$ which has the sets $\{f \neq 0\} \cap\{g=0\}$ $(f, g \in A)$ as a basis of open sets.

Hence $C_{M}=$ DENSE $^{\text {con }}$, in particular $C_{M} \models$ DENSE.

8. Let

$$
\mathrm{pt}^{*}(x):=\operatorname{pt}(x) \wedge \forall y x \neq y^{2} \wedge x \neq-y^{2} .
$$

Hence, as $A$ is a real closed ring, a point function $f$ of $A$ is in $\operatorname{pt}^{*}(A)$ if and only if $f$ is neither everywhere positive nor everywhere negative. We call the elements of $\mathrm{pt}^{*}(A)$ the sign changing point functions. Let LINE be the sentence

$$
\mathrm{LINE}:=\forall x\left(\operatorname{pt}(x) \rightarrow \exists y x \succ y \wedge \mathrm{pt}^{*}(y)\right) .
$$

Hence by definition, $A$ satisfies LINE if and only if each point of $A$ is the Jacobson radical of a point function which changes sign. This for example holds in the ring $C_{M}$ and resembles the shape of $M$ as a line. Note that in the ring of continuous semi-algebraic maps $M^{2} \rightarrow M$ no point function changes sign.

9. For $f, g \in A$ we define $f \| g$ if and only if $f, g \in \mathrm{pt}^{*}(A)$ and both $f^{+}+g^{+}, f^{-}+g^{-}$are zero-divisors. Since $\mathrm{pt}^{*}(A)$ is defined by the same formula for all real closed rings, there is a first order formula $\varphi(x, y)$ in the 
language of rings such that for every real closed ring $A$ and all $f, g \in A$ we have

$$
f \| g \Leftrightarrow A=\varphi(f, g) .
$$

Let $f, g \in C_{M}$ be point functions which change sign. Then a straightforward calculation shows that $f \| g$ if and only if $f$ is strictly increasing in a neighbourhood of its zero and $g$ is strictly increasing in a neighbourhood of its zero, or $f$ is strictly decreasing in a neighbourhood of its zero and $g$ is strictly decreasing in a neighbourhood of its zero.

10. Let $f \in \mathrm{pt}^{*}\left(C_{M}\right)$. Then for every $g \in \mathrm{pt}^{*}\left(C_{M}\right)$ we have $f \| g \Leftrightarrow$ $f \nVdash-g$, hence there is a unique $\varepsilon(f, g) \in\{ \pm 1\} \subseteq C_{M}$ such that $f \| \varepsilon(f, g) \cdot g$ and there is a first order formula in the language of rings which defines the graph of $\varepsilon$ in $C_{M}^{3}$. We define a preorder $\sqsubseteq_{f}$ on $\mathrm{pt}^{*}\left(C_{M}\right)$ as follows. We say $g \sqsubseteq f h$ if and only if $(\varepsilon(f, h) \cdot h)^{+}+(-\varepsilon(f, g) \cdot g)^{+}$is a non-zero-divisor.

It turns out that $g \succ h$ if and only if $g \sqsubseteq_{f} h$ and $h \sqsubseteq_{f} g$. Moreover $\mathrm{pt}^{*}\left(C_{M}\right) / \succ$ (which is "equal" to $\operatorname{Max}_{\mathrm{pt}}\left(C_{M}\right)$ ) equipped with the induced order of $\sqsubseteq_{f}$ is order isomorphic to $M$ or to $M^{\text {opp }}$ (according as $f$ is strictly increasing or strictly decreasing in a neighbourhood of its zero).

Moreover there is a first order formula $\psi(x, y, z)$ in the language of rings such that for all $f, g, h \in C_{M}$ we have

$$
g \sqsubseteq_{f} h \Leftrightarrow C_{M}=\psi(f, g, h) .
$$

We write $y \sqsubseteq_{x} z$ for this formula and we write $y \sqsubset_{x} z$ for $y \sqsubseteq_{x} z \wedge \neg\left(z \sqsubseteq_{x} y\right)$.

11. The sentence

$$
\begin{aligned}
& \forall x \mathrm{pt}^{*}(x) \rightarrow\left[\left[\forall u, v, w \mathrm{pt}^{*}(u)\right.\right. \wedge \mathrm{pt}^{*}(v) \wedge \mathrm{pt}^{*}(w) \rightarrow \\
&\left.\left(u \sqsubseteq_{x} v \wedge v \sqsubseteq_{x} w \rightarrow u \sqsubseteq_{x} w\right) \wedge\left(u \sqsubseteq_{x} v \vee v \sqsubseteq_{x} u\right)\right] \\
&\left.\wedge\left[\forall u, v \mathrm{pt}^{*}(u) \wedge \mathrm{pt}^{*}(v) \rightarrow u \succ v \leftrightarrow\left(u \sqsubseteq_{x} v \wedge v \sqsubseteq_{x} u\right)\right]\right]
\end{aligned}
$$

holds in $C_{M}$; it says that for each sign changing point function $f$, the relation $\sqsubseteq_{f}$ defines a total semi-order on the set of sign changing point functions, and the induced total order is in 1-1 correspondence with those points that are generated by a sign changing point function.

Clearly there is also a sentence DLO in the language of rings which says that all these total orderings are dense without endpoints.

12. Suppose $C(X) \models$ DENSE $\wedge$ LINE $\wedge$ DLO. Let $f \in \operatorname{pt}^{*}(X)$. Let $S:=\{x \in X \mid\{x\}$ is a zero set $\}$. Then $S$ is dense in $X$ and the formula $y \sqsubseteq_{f} z$ defines a total semi-order on the set of sign changing point functions of $C(X)$. Moreover, the underlying set of the induced total order is in 1-1 correspondence with $S$.

Hence if we identify an element $s$ of $S$ with the set of point functions that vanish at $s$, then $S$ is totally ordered by $\sqsubseteq_{f}$ and this order is dense without endpoints. 
13. Let BUMPS be the sentence in the language of rings which says the following: "For all sign changing point functions $f, g, h_{1}, h_{2}$ with $h_{1} \sqsubset_{f} g$ $\sqsubset_{f} h_{2}$ there is $F$ such that

(i) $0 \leq F \leq 1$,

(ii) $F \nprec g$,

(iii) for all sign changing point functions $h^{\prime}$ with $F \nprec h^{\prime}$ we have $h_{1} \sqsubset_{f}$ $h^{\prime} \sqsubset_{f} h_{2} . "$

Intuitively, BUMPS says that with respect to $\sqsubseteq_{f}$, for every point (given as the zero of $g$ ) in an open interval (whose endpoints are given by the zeros of $h_{1}$ and $h_{2}$ ), there is some $F, 0 \leq F \leq 1$, which vanishes outside this interval and which does not vanish at the given point. Clearly BUMPS holds in $C_{M}$.

14. Let $\operatorname{DEFCOMPLETE}_{1}(F, f, h)$ be the formula in the language of rings which says the following: " $f$ is a sign changing point function and $h$ is the smallest sign changing point function with respect to $\sqsubseteq_{f}$ such that

(i) $f \sqsubseteq_{f} h$,

(ii) either $F \prec h^{\prime}$ for all sign changing point functions $h^{\prime}$ with $h \sqsubseteq_{f} h^{\prime}$, or $F \nprec h^{\prime}$ for all such $h^{\prime}$."

Intuitively, $\operatorname{DEFCOMPLETE}_{1}(F, f, h)$ says that with respect to $\sqsubseteq_{f}$, the zero of $h$ is the supremum of all points greater than or equal to the zero of $f$ for which $F$ changes sign from 0 to $\neq 0$ or from $\neq 0$ to 0 . In $C_{M}$, $\operatorname{DEFCOMPLETE}_{1}(F, f, h)$ in fact says precisely this.

Let

DEFCOMPLETE $:=\forall F, f \mathrm{pt}^{*}(f) \rightarrow \exists ! h \operatorname{DEFCOMPLETE}_{1}(F, f, h)$. Then $C_{M}=$ DEFCOMPLETE, by o-minimality of $M$.

15. We now encode in $C_{M}$ the fact that in an o-minimal structure $M$, unary functions do not change sign close to the left hand side of any given point.

Let $\operatorname{NOSIGNCHANGE}_{1}\left(F, f, h_{0}, h\right)$ be the formula in the language of rings which says the following: " $f, h, h_{0}$ are sign changing point functions with $h_{0} \sqsubset_{f} h$, and either $F \prec h^{\prime}$ for all sign changing point functions $h^{\prime}$ with $h_{0} \sqsubseteq_{f} h^{\prime} \sqsubset_{f} h$, or $F \nprec h^{\prime}$ for all such $h^{\prime}$."

Hence NOSIGNCHANGE ${ }_{1}\left(F, f, h_{0}, h\right)$ says that the function $F$ either is constantly zero on the half open interval $\left[h_{0}, h\right)$ with respect to $\sqsubseteq_{f}$, or does not have any zeros in that interval.

Let NOSIGNCHANGE be the sentence

$$
\forall F, f, h\left[\mathrm{pt}^{*}(f) \wedge \mathrm{pt}^{*}(h) \rightarrow \exists h_{0} \operatorname{NOSIGNCHANGE}_{1}\left(F, f, h_{0}, h\right)\right] .
$$

Again, by o-minimality of $M, C_{M}=$ NOSIGNCHANGE. 
16. The sentence

\section{$\mathrm{DENSE} \wedge \mathrm{LINE} \wedge \mathrm{DLO} \wedge \mathrm{BUMPS} \wedge \mathrm{DEFCOMPLETE} \wedge$ NOSIGNCHANGE} holds in every ring $C_{M}$ but in no $\operatorname{ring} C(X)$.

Indeed, we have already seen during the definitions of the subsentences that $C_{M}$ satisfies this conjunction. Let $X$ be a Tikhonov space and suppose the sentence

\section{$\mathrm{DENSE} \wedge \mathrm{LINE} \wedge \mathrm{DLO} \wedge \mathrm{BUMPS} \wedge \mathrm{DEFCOMPLETE}$}

holds in $C(X)$. We shall construct functions $F, f, h$ in $C(X)$ which violate the property stated in the definition of NOSIGNCHANGE.

Since $C(X) \models$ DENSE $\wedge$ LINE there is a sign changing point function $f \in C(X)$. Let $g_{i} \in C(X)(i \in \mathbb{N})$ be sign changing point functions with $f \sqsubset_{f} g_{1} \sqsubset_{f} g_{2} \sqsubset_{f} \cdots$ such that the sequence $\left(g_{i}\right)$ is bounded in the preorder $\sqsubseteq_{f}$ defined on $\mathrm{pt}^{*}(C(X))$; such $g_{i}$ exist since $C(X) \models \mathrm{DLO}$. Since $C(X) \models$ BUMPS there are $F_{i} \in C(X)$ with $0 \leq F_{i} \leq 1$ such that $F_{i} \nprec g_{2 i}$ and such that for all $h \in \mathrm{pt}^{*}(C(X))$ with $F_{i} \nprec h$ we have $g_{2 i-1} \sqsubset_{f} h \sqsubset_{f} g_{2 i+1}$.

Intuitively, $\left(g_{i}\right)$ is a strictly increasing sequence of (representatives of) points (with respect to $\sqsubseteq_{f}$ ) and the $F_{i}$ are functions from $C(X)$ which are nonzero in $g_{2 i}$ so that every nonzero point of $F_{i}$ is in the open interval $\left(g_{2 i-1}, g_{2 i+1}\right)$ (in particular, each $F_{i}$ vanishes on each $\left.g_{2 k+1}\right)$.

Since each $F_{i}$ satisfies $0 \leq F_{i} \leq 1$, the function $F:=\sum_{i \in \mathbb{N}} 2^{-i} F_{i}$ is continuous on $X$. Moreover, by the choice of the $F_{i}$, for every $h \in \mathrm{pt}^{*}(C(X))$ with $g_{j} \sqsubseteq_{f} h(j \in \mathbb{N})$ we have $F_{i} \prec h$. Thus $F_{i}(x)=0$, where $x$ is the zero of $h$ (cf. item 12). Thus also $F(x)=0$, which in turn means $F \prec h$.

Since $C(X)=$ DEFCOMPLETE and $\left(g_{i}\right)$ is a bounded sequence with respect to $\Xi_{f}$, there is a minimal $h \in \mathrm{pt}^{*}(C(X))$ such that $F \prec h^{\prime}$ for all $h \sqsubseteq_{f} h^{\prime} \in \mathrm{pt}^{*}(C(X))$. By construction of $F, h$ is the supremum of the sequence $\left(g_{i}\right)$ with respect to $\sqsubseteq_{f}$.

Therefore, if $\mathrm{pt}^{*}(C(X)) \ni h_{0} \sqsubset_{f} h$, then there is some $i \in \mathbb{N}$ with $h_{0} \sqsubseteq_{f} g_{2 i} \sqsubseteq_{f} h$, hence also $h_{0} \sqsubseteq_{f} g_{2 i+1} \sqsubseteq_{f} h$.

But $F \nprec g_{2 i}$ and $F \prec g_{2 i+1}$, and this shows that $F, f, h$ violate NOSIGNCHANGE.

Hence the sentence stated in item 16 has the property described at the beginning of this section. Actually one can show that there is a sentence $\varphi$ in the language of rings which holds in every ring $A$ of continuous definable functions $X \rightarrow M$ for every o-minimal expansion of a real closed field $M$ and every definable subset $X$ of dimension $>0$, but which does not hold in any $C(X)$ (the reason is that each such set contains a definable curve germ and then it is possible to interpret $C_{M}$ in $A$ and with this interpretation we can code the sentence in item 16). 


\section{Super real closed rings: Definition and basic properties}

(5.1) Definition.

(a) Let $\mathscr{L}_{\Upsilon}$ be the first order language extending the language $\{+,-, \cdot$, $0,1\}$ of rings which has in addition an $n$-ary function symbol $\underline{F}$ for every continuous function $F: \mathbb{R}^{n} \rightarrow \mathbb{R}$ and every $n \in \mathbb{N}_{0}$.

(b) Let $T_{\Upsilon}$ be the $\mathscr{L}_{\Upsilon}$-theory with the following axioms:

1. The axioms of a commutative unital ring (with 1 ) in the language $\{+,-, \cdot, 0,1\}$.

2. The axiom $\forall x y( \pm(x, y)=x+y \wedge:(x, y)=x \cdot y \wedge \underline{\operatorname{id}}(x)=x \wedge$ $\overline{-}(x)=-x \wedge \underline{1}(x)=1 \wedge \underline{0}(x)=1)$. Hence the symbols from the language of rings have the same meaning as the corresponding symbols when reintroduced in $\mathscr{L}_{\Upsilon}$ as symbols naming continuous functions.

3. All the sentences

$$
\forall \bar{x} \underline{F}\left(\underline{f_{1}}(\bar{x}), \ldots, \underline{f_{n}}(\bar{x})\right)=\underline{F \circ\left(f_{1}, \ldots, f_{n}\right)}(\bar{x})
$$

for $F \in C\left(\mathbb{R}^{n}\right)$ and $f_{1}, \ldots, f_{n} \in C\left(\bar{R}^{\bar{x}}\right)$.

The models of $T_{\Upsilon}$ are called super real closed rings. We shall denote the functions $\underline{F}^{A}$ of a super real closed ring by $F_{A}\left(F \in C\left(\mathbb{R}^{n}\right)\right)$; one might think of $F_{A}$ as the scalar extension of $F$ to $A$. If it is clear how the $F_{A}$ are defined we even drop the subscript $A$ and write $F$ again for $F_{A}$.

If $A$ is a super real closed ring and a local ring, a domain, a field, etc. then $A$ is called a super real closed local ring, a super real closed domain, a super real closed field, etc.

Observe that the null ring is also considered as a super real closed ring.

Note that if $F \in C\left(\mathbb{R}^{n}\right)$ is considered as the function $G \in C\left(\mathbb{R}^{n} \times\right.$ $\left.\mathbb{R}^{k}\right)$ defined by $G(\bar{x}, \bar{y}):=F(\bar{x})$, then $T_{\Upsilon} \vdash \forall x_{1}, \ldots, x_{n}, y_{1}, \ldots, y_{k} \underline{F}(\bar{x})=$ $\underline{G}(\bar{x}, \bar{y})$, since $G=F \circ p$ for the projection $p: \mathbb{R}^{n} \times \mathbb{R}^{k} \rightarrow \mathbb{R}^{n}$.

The natural examples of super real closed rings are rings of continuous functions:

(5.2) Definition. If $X$ is a Tikhonov space, then we expand $C(X)$ to a super real closed ring, denoted by $C(X)_{\Upsilon}$, via

$$
\underline{F}^{C(X)_{\Upsilon}}\left(f_{1}, \ldots, f_{n}\right):=F \circ\left(f_{1}, \ldots, f_{n}\right) .
$$

In particular $\mathbb{R}_{\Upsilon}$ is the super real closed field expanding the ring $\mathbb{R}$, where $\underline{F}^{\mathbb{R}_{\Upsilon}}=F$.

(5.3) Definition. A homomorphism between $\mathscr{L}_{\Upsilon \text {-structures is called }}$ a super homomorphism. An $\mathscr{L}_{\Upsilon}$-substructure of an $\mathscr{L}_{\Upsilon}$-structure is called a super substructure. 
(5.4) Definition. Since $T_{\Upsilon}$ is axiomatized by sentences of the form $\forall \bar{x} t_{1}(\bar{x})=t_{2}(\bar{x})$ for $\mathscr{L}_{\Upsilon}$-terms $t_{1}, t_{2}, T_{\Upsilon}$ has term models over any set of constants $C$ (cf. [Ho, Section 9], where term models are called "term algebras"). We write $\operatorname{tm}_{\Upsilon}(C)$ for the term model of super real closed rings over a set $C$ of constants.

Recall that $\operatorname{tm}_{\Upsilon}(C)$ is the following structure: Let $\mathscr{L}^{*}$ be the language $\mathscr{L}_{\Upsilon}$ together with a new constant for every element in $C$. Then the universe of $\operatorname{tm}_{\Upsilon}(C)$ is the set of all constant terms in the language $\mathscr{L}^{*}$ modulo the equivalence relation $t \sim t^{\prime} \Leftrightarrow T_{\Upsilon} \vdash t=t^{\prime}$. The function symbols $F$ from $\mathscr{L}_{\Upsilon}$ are interpreted as $\underline{F}^{\operatorname{tm}_{\Upsilon}(C)}\left(t_{1} / \sim, \ldots, t_{n} / \sim\right):=F\left(t_{1}, \ldots, t_{n}\right) / \sim$.

Also recall that $\operatorname{tm}_{\Upsilon}(C)$ is characterized by the following property: for every super real closed ring $A$ and every map $f: C \rightarrow A$, there is a unique super homomorphism $\operatorname{tm}_{\Upsilon}(C) \rightarrow A$ extending $f$. In other words, $\operatorname{Hom}_{\Upsilon}\left(\operatorname{tm}_{\Upsilon}(C), A\right)=A^{C}$. In particular $\operatorname{tm}_{\Upsilon}(C)$ is uniquely determined up to an $\mathscr{L}_{\Upsilon}$-isomorphism by the cardinality of $C$.

(5.5) Theorem.

(i) $T_{\Upsilon}$ is axiomatized by sentences of the form $\forall \bar{x} t_{1}(\bar{x})=t_{2}(\bar{x})$, where $t_{1}, t_{2}$ are $\mathscr{L}_{\Upsilon}$-terms. Moreover, for every $\mathscr{L}_{\Upsilon}$-term $t(\bar{x})$, there is some $F \in C\left(\mathbb{R}^{\bar{x}}\right)$ with $T_{\Upsilon} \vdash \forall \bar{x} t(\bar{x})=F(\bar{x})$. In particular the category of all models of $T_{\Upsilon}$ together with super homomorphisms is a variety in the sense of universal algebra.

(ii) $\mathbb{R}_{\Upsilon}$ is the term model of $T_{\Upsilon}$, in particular for every super real closed ring $A$, there is a unique super homomorphism $\mathbb{R}_{\Upsilon} \rightarrow A$.

(iii) $T_{\Upsilon}$ is an extension of RCR, hence every model of $T_{\Upsilon}$ is a real closed ring (cf. (2.1)).

(iv) $T_{\Upsilon} \vdash \forall \bar{x} \underline{F}(\bar{x})=0 \rightarrow \underline{G}(\bar{x})=0$ for all $F, G \in C\left(\mathbb{R}^{n}\right)$ with $\{F=$ $0\} \subseteq\{G=0\}$.

(v) $T_{\Upsilon} \cup$ "domains" is the universal theory $\operatorname{Th}\left(\mathbb{R}_{\Upsilon}\right)_{\forall}$ of $\mathbb{R}_{\Upsilon}$.

Proof. (i) and (ii) hold by definition of $T_{\Upsilon}$.

(iii) holds by (our) definition of real closed rings.

(iv) By (3.7), $\mathbb{R}^{n}$ has computable $z$-radicals. Hence $\{F=0\} \subseteq\{G=0\}$ implies $G=g \cdot s \circ(F \cdot f)$ for some $f, g \in C\left(\mathbb{R}^{n}\right)$ and some $s \in \Upsilon$. Therefore $T_{\Upsilon} \vdash \forall \bar{x} \underline{G}(\bar{x})=g \cdot \underline{s}(\underline{F}(\bar{x}) \cdot \underline{f}(\bar{x}))$. Since $T_{\Upsilon} \vdash \underline{s}(0)=0$ we get the claim.

(v) Since $\mathbb{R}_{\Upsilon}$ is a domain and a model of $T_{\Upsilon}$ we have $T_{\Upsilon} \cup$ "domains" $\subseteq$ $\operatorname{Th}\left(\mathbb{R}_{\Upsilon}\right)_{\forall}$. Conversely, let $\varphi(\bar{x})$ be a quantifier free $\mathscr{L}_{\Upsilon}$-formula such that $\mathbb{R}_{\Upsilon}=\forall \bar{x} \varphi(\bar{x})$. We have to show that in every super real closed ring $A$ which is a domain we have $A=\forall \bar{x} \varphi(\bar{x})$. Let $\bar{a} \in A^{\bar{x}}$. We have to show $A=\varphi(\bar{a})$. Now $\varphi$ is a finite conjunction of finite disjunctions of formulas of the form $t_{1}(\bar{x})=t_{2}(\bar{x})$ or $t_{1}(\bar{x}) \neq t_{2}(\bar{x})$, where $t_{1}, t_{2}$ are $\mathscr{L}_{\Upsilon}$-terms. By (i) we may assume that these atomic parts are of the form $\underline{F}(\bar{x})=0$ or $\underline{F}(\bar{x}) \neq 0$ 
for some $F \in C\left(\mathbb{R}^{\bar{x}}\right)$. So in order to prove $A=\varphi(\bar{a})$ we may assume that $\varphi$ is of the form $\bigvee_{i} \underline{F}_{i}(\bar{x})=0 \vee \bigvee_{j} G_{j}(\bar{x}) \neq 0$. Since $A$ is a domain it is enough to show $A=\prod_{i} \underline{F}_{i}(\bar{a})=0 \vee \sum_{j} \underline{G}_{j}{ }^{2}(\bar{a}) \neq 0$. Since $\mathbb{R}_{\Upsilon} \models \forall \bar{x} \varphi(\bar{x})$ we know $\mathbb{R}_{\Upsilon} \models \forall \bar{x} \sum_{j} \underline{G j}^{2}(\bar{x})=0 \rightarrow \prod_{i} \underline{F}_{i}(\bar{x})=0$. So by (iv) we have $T_{\Upsilon} \vdash \forall \bar{x} \sum_{j} \underline{G}_{j}^{2}(\bar{x})=0 \rightarrow \prod_{i} \underline{F_{i}}(\bar{x})=0$ as desired.

(5.6) CoRollary. If $A$ is a super real closed domain, then the super real closed ring $\mathbb{R}_{\Upsilon}$ is existentially closed in $A$.

Proof. This is a reformulation of $(5.5)(\mathrm{v})$.

By (5.5)(i), a super substructure $A$ of a super real closed ring $B$ is again a super real closed ring. We then call $A$ a super real closed subring.

(5.7) Lemma. Let $f: A \rightarrow B$ be an injective super homomorphism between super real closed rings. Then $f$ is an $\mathscr{L}_{\Upsilon}$-embedding, i.e. for every quantifier free $\mathscr{L}_{\Upsilon}$-formula $\varphi(\bar{x})$ and all $\bar{a} \in A^{\bar{x}}$ we have $A=\varphi(\bar{a}) \Leftrightarrow B \models$ $\varphi(f(\bar{a}))$. In particular $f$ is an $\mathscr{L}_{\Upsilon}$-isomorphism if $f$ is bijective.

Proof. We may assume that $\varphi(\bar{x})$ is of the form $\underline{F}(\bar{x})=0$ for some $F \in C\left(\mathbb{R}^{\bar{x}}\right)$. If $A=\underline{F}(\bar{a})=0$, then $B \models \underline{F}(f(\bar{a}))=0$, since $f$ is a super homomorphism. Conversely, if $A \models \underline{F}(\bar{a}) \neq 0$, then as $f$ is injective we have $f(\underline{F}(\bar{a})) \neq 0$. Since $f(\underline{F}(\bar{a}))=\underline{F}(f(\bar{a}))$ we get the lemma.

(5.8) Proposition. If $\mathcal{C}$ is a finite set of cardinality $n$, then the term model $\operatorname{tm}_{\Upsilon}(\mathcal{C})$ is $C\left(\mathbb{R}^{n}\right)$. If $\mathcal{C}$ is infinite, then $\operatorname{tm}_{\Upsilon}(\mathcal{C})$ is the following subring of $C\left(\mathbb{R}^{\mathcal{C}}\right)$ : for each finite subset $\mathcal{E} \subseteq \mathcal{C}$ let $C_{\mathcal{E}}$ be the subring of $C\left(\mathbb{R}^{\mathcal{C}}\right)$ induced by the projection $\mathbb{R}^{\mathcal{C}} \rightarrow \mathbb{R}^{\mathcal{E}}$; then $\operatorname{tm} \Upsilon(\mathcal{C})=\bigcup_{\mathcal{E} \subseteq \mathcal{C} \text {, finite }} C_{\mathcal{E}}$.

Proof. Straightforward.

By the shape of the axioms of $T_{\Upsilon}$ we get many constructions known from commutative algebra for free also in the category of super real closed rings and super homomorphisms. In order not to inflate the text we will use them ad hoc whenever needed and refer to basic model theory for the justification. For example, in (5.8) above, the free super real closed ring of cardinality card $\mathcal{C}$ is introduced. Similarly, there is a free super real closed ring of cardinality $\mathcal{C}$ over every given super real closed ring. Four other constructions obtained from the axiomatization should be mentioned right now:

The category of super real closed rings and super homomorphisms has direct limits and fibre sums as well as inverse limits and fibre products. Moreover the underlying ring of a direct limit, an inverse limit and a fibre product of super real closed rings is also the direct limit, the inverse limit and the fibre product in the category of commutative rings, respectively. All 
this follows via routine checking from the definitions of the constructions in both categories.

On the other hand, the relation between the fibre sum of super real closed rings and the tensor product of these rings is a subtle matter. For example, given super real closed ring extensions $A \subseteq B, C$, it is an open problem whether the natural ring homomorphism $f$ from the real closure (cf. (2.17)) of the tensor product $B \otimes_{A} C$ in the category of rings to the fibre sum $D$ of $B, C$ over $A$ in the category of super real closed rings is injective (specializing $A, B$ and $C$ to super real closed fields, this question asks whether super real closed fields have the amalgamation property). It is also unclear whether the image of $f$ generates the super real closed ring $D$.

6. $\Upsilon$-ideals. Recall from (3.1) that an ideal $\mathfrak{a}$ of $C(X)$ is called $\Upsilon$-radical if $s \circ f \in \mathfrak{a}$ for all $f \in \mathfrak{a}$ and all $s \in \Upsilon$.

(6.1) Definition. An ideal $\mathfrak{a}$ of a super real closed $\operatorname{ring} A$ is called an $\Upsilon$-ideal or an $\Upsilon$-radical ideal if $s_{A}(\mathfrak{a}) \subseteq \mathfrak{a}$ for all $s \in \Upsilon$.

We will show that $\Upsilon$-radical ideals are precisely the kernels of super homomorphisms in (6.3) below. As a preparation we need:

(6.2) Lemma. If $F: \mathbb{R}^{n} \rightarrow \mathbb{R}$ is continuous, then there is some $s \in \Upsilon$ with

$$
s\left(F\left(x_{1}, \ldots, x_{n}\right)-F\left(y_{1}, \ldots, y_{n}\right)\right) \in\left(x_{1}-y_{1}, \ldots, x_{n}-y_{n}\right),
$$

where $\left(x_{1}-y_{1}, \ldots, x_{n}-y_{n}\right)$ denotes the ideal generated by $x_{1}-y_{1}, \ldots, x_{n}-y_{n}$ in $C\left(\mathbb{R}^{n} \times \mathbb{R}^{n}\right)$.

Proof. Let $G \in C\left(\mathbb{R}^{n} \times \mathbb{R}^{n}\right)$ be defined by $G(\bar{x}, \bar{y}):=|F(\bar{x})-F(\bar{y})|$. Let $H \in C\left(\mathbb{R}^{n} \times \mathbb{R}^{n}\right)$ be defined by $H(\bar{x}, \bar{y}):=\sum_{i=1}^{n}\left(x_{i}-y_{i}\right)^{2}$. Then $\{H=0\} \subseteq$ $\{G=0\}$ and since $\mathbb{R}^{n} \times \mathbb{R}^{n}$ has computable $z$-radicals, there is some $s \in \Upsilon$ such that $H$ divides $s \circ G$. Hence $s \circ G \in H \cdot C\left(\mathbb{R}^{n} \times \mathbb{R}^{n}\right) \subseteq\left(x_{1}-y_{1}, \ldots\right.$, $\left.x_{n}-y_{n}\right)$.

(6.3) Theorem. Let $\mathfrak{a}$ be an ideal of a super real closed ring A. Then $\mathfrak{a}$ is $\Upsilon$-radical if and only if $\mathfrak{a}$ is the kernel of a super homomorphism $A \rightarrow B$ for some super real closed ring $B$. In this case, there is a unique expansion of the ring $A / \mathfrak{a}$ to a super real closed ring such that the residue map $A \rightarrow A / \mathfrak{a}$ is a super homomorphism.

Proof. If there is a super homomorphism $\varphi: A \rightarrow B$ with $\mathfrak{a}=\operatorname{Ker} \varphi$, then for $f \in \mathfrak{a}$ and $s \in \Upsilon$ we have $\varphi\left(s_{A}(f)\right)=s_{B}(\varphi(f))=s_{B}(0)=0$, so $s_{A}(f) \in \mathfrak{a}$. Thus $\mathfrak{a}$ is $\Upsilon$-radical.

Conversely, suppose $s_{A}(\mathfrak{a}) \subseteq \mathfrak{a}$ for all $s \in \Upsilon$.

Claim. If $F \in C\left(\mathbb{R}^{n}\right)$ and $f_{1}, g_{1}, \ldots, f_{n}, g_{n} \in A$ with $f_{i}-g_{i} \in \mathfrak{a}(1 \leq$ $i \leq n)$ then $F_{A}\left(f_{1}, \ldots, f_{n}\right)-F_{A}\left(g_{1}, \ldots, g_{n}\right) \in \mathfrak{a}$. 
Proof. Let $\varphi$ be the super homomorphism $C\left(\mathbb{R}^{n} \times \mathbb{R}^{n}\right) \rightarrow A$ which maps the projections $x_{i}$ to $f_{i}$ and $y_{i}$ to $g_{i}(1 \leq i \leq n)$. Let $\mathfrak{b}:=\varphi^{-1}(\mathfrak{a})$. Then, as $s_{A}(\mathfrak{a}) \subseteq \mathfrak{a}$ for all $s \in \Upsilon$, we also have $s(\mathfrak{b}) \subseteq \mathfrak{b}$ for all $s \in \Upsilon$. By (6.2) there is some $s \in \Upsilon$ with $s\left(F\left(x_{1}, \ldots, x_{n}\right)-F\left(y_{1}, \ldots, y_{n}\right)\right) \in$ $\left(x_{1}-y_{1}, \ldots, x_{n}-y_{n}\right)$. By assumption $\left(x_{1}-y_{1}, \ldots, x_{n}-y_{n}\right) \subseteq \mathfrak{b}$. Since $\mathfrak{b}$ is $\Upsilon$ radical we get $F\left(x_{1}, \ldots, x_{n}\right)-F\left(y_{1}, \ldots, y_{n}\right) \in \mathfrak{b}=\varphi^{-1}(\mathfrak{a})$, so $F_{A}\left(f_{1}, \ldots, f_{n}\right)$ $-F_{A}\left(g_{1}, \ldots, g_{n}\right)=\varphi\left(F\left(x_{1}, \ldots, x_{n}\right)-F\left(y_{1}, \ldots, y_{n}\right)\right) \in \mathfrak{a}$.

This proves the claim and we may define, for all $F \in C\left(\mathbb{R}^{n}\right)$ and all $f_{1}, \ldots, f_{n} \in \mathfrak{a}$,

$$
F_{A / \mathfrak{a}}\left(f_{1} \bmod \mathfrak{a}, \ldots, f_{n} \bmod \mathfrak{a}\right):=F_{A}\left(f_{1}, \ldots, f_{n}\right) \bmod \mathfrak{a} .
$$

With this interpretation of the function symbols from $\mathscr{L}_{\Upsilon}, A / \mathfrak{a}$ becomes an $\mathscr{L}_{r}$-structure and the residue map $A \rightarrow A / \mathfrak{a}$ is a super homomorphism. Clearly $A / \mathfrak{a}$ is a super real closed ring and it is the unique expansion of $A / \mathfrak{a}$ to a super real closed ring such that the residue map $A \rightarrow A / \mathfrak{a}$ is a super homomorphism.

(6.4) Definition. If $\mathfrak{a}$ is an ideal of a super real closed ring, then the smallest $\Upsilon$-ideal containing $\mathfrak{a}$ is called the $\Upsilon$-radical of $\mathfrak{a}$. We denote the $\Upsilon$-radical of $\mathfrak{a}$ by $\sqrt[\Upsilon]{\mathfrak{a}}$.

(6.5) Remark. We shall now generalize results from [Tr1, Section 5] to super real closed rings, in particular we compute the $\Upsilon$-radical of an ideal of a super real closed ring. In proving these generalizations we use mostly the following strategy.

Any super real closed ring $A$ is the union of the finitely generated super real closed subrings of $A$. These are subrings $B$ of $A$ which are the $\mathscr{L}_{\Upsilon^{-}}$ substructures of $A$ generated by a finite subset of $A$. Each of these subrings is of the form $C\left(\mathbb{R}^{n}\right) / I$ for some $\Upsilon$-radical ideal $I$ of $C\left(\mathbb{R}^{n}\right)$ (since $\mathbb{R}^{n}$ has computable $z$-radicals, $I$ is even a $z$-ideal).

Therefore we proceed by proving our statements first for quotients of rings $C(X)$ by $\Upsilon$-radical ideals, and then for unions of finitely generated super real closed rings.

In doing this, we shall constantly use the observation that the preimage of an $\Upsilon$-radical ideal under a super homomorphism is again $\Upsilon$-radical (which follows immediately from the definition of $\Upsilon$-radical ideal, cf. (6.1)). Here is an example:

If $\mathfrak{a}$ is an ideal of a super real closed ring A, then there is a largest $\Upsilon$-radical ideal contained in $\mathfrak{a}$.

Proof.

Claim. The assertion holds if $A=C(X) / I$ for some $\Upsilon$-radical ideal $I$ of $C(X)$. 
By (6.3), the residue map $C(X) \rightarrow A$ induces a bijection between the $\Upsilon$-radical ideals of $A$ and the $\Upsilon$-radical ideals of $C(X)$ containing $I$. Since for every ideal $\mathfrak{b}$ of $C(X)$ there is a largest $\Upsilon$-radical ideal of $C(X)$ contained in $\mathfrak{b}$ (cf. $[\operatorname{Tr} 1,(3.4)]$ ) the same property also holds for all ideals of $A$. This shows the claim.

Now we get the assertion as follows. $A$ is the union of the finitely generated super real closed subrings of $A$. By the claim, for every ideal $\mathfrak{b}$ of such a ring $B$ there is a largest $\Upsilon$-radical ideal $\mathfrak{b}^{\Upsilon}$ of $B$ contained in $\mathfrak{b}$. Now we see that

$$
\bigcup_{B \subseteq A \text {, fin. gen. }}(\mathfrak{a} \cap B)^{\Upsilon}
$$

is the largest $\Upsilon$-radical ideal of $A$ contained in $\mathfrak{a}$.

So we may define

(6.6) Definition. Let $\mathfrak{a}$ be an ideal of a super real closed ring $A$. Then there is a largest $\Upsilon$-radical ideal contained in $\mathfrak{a}$, which we denote by $\mathfrak{a}^{\Upsilon}$.

(6.7) Proposition. If $\mathfrak{a}$ is an ideal of the super real closed ring $A$, then

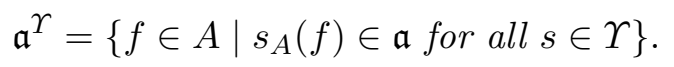

If $\Upsilon_{0} \subseteq \Upsilon$ is a set of generalized root functions (cf. (3.2)), then

$$
\mathfrak{a}^{\Upsilon}=\left\{f \in \mathfrak{a} \mid s_{0, A}(f), s_{0, A}(-f) \in \mathfrak{a} \text { for all } s_{0} \in \Upsilon_{0}\right\} .
$$

Proof. Follows from (3.4), using the strategy explained in (6.5).

(6.8) Proposition. For all ideals $\mathfrak{a}, \mathfrak{b}$ of a super real closed ring $A$ we have $\sqrt[r]{\mathfrak{a}+\mathfrak{b}}=\sqrt[r]{\mathfrak{a}}+\sqrt[r]{\mathfrak{b}}$

Proof. Follows from [Tr1, (5.8)], using the strategy explained in (6.5).

Recall that for any ring extension $A \subseteq B$, the induced map Spec $B \rightarrow$ $\operatorname{Spec} A$ is dominant, i.e. the image is dense, in other words every minimal prime ideal of $A$ is in the image of this map.

(6.9) Proposition. If $A$ is a super real closed ring and $\mathfrak{p}$ is a prime ideal of $A$, then $\mathfrak{p}^{\Upsilon}$ is a prime ideal of $A$. In particular the minimal prime ideals of $A$ are $\Upsilon$-radical.

Proof. First let $A$ be a term model of super real closed rings (cf. (5.4)). Then $A$ is a super real closed subring of $C\left(\mathbb{R}^{T}\right)$ for some set $T$. Since the minimal primes of $C\left(\mathbb{R}^{T}\right)$ are $\Upsilon$-radical (they are even $z$-radical) and Spec $C\left(\mathbb{R}^{T}\right) \rightarrow \operatorname{Spec} A$ is dominant, each minimal prime ideal of $A$ is $\Upsilon$ radical. Therefore, if $\mathfrak{p}$ is a prime ideal of the term model $A$, then $\mathfrak{p}$ contains an $\Upsilon$-ideal $\mathfrak{q}$ which is prime. Since $\mathfrak{q} \subseteq \mathfrak{p}^{\Upsilon}$ and $\mathfrak{p}^{\Upsilon}$ is a radical ideal of $A$, we conclude that $\mathfrak{p}^{\Upsilon}$ is prime as well.

Now let $A$ be an arbitrary super real closed ring. Then $A=B / I$ for some term model $B$ of super real closed rings and some $\Upsilon$-radical ideal $I$ 
of $B$. Since the residue map $B \rightarrow A$ induces a bijection between the $\Upsilon$ radical ideals of $A$ and the $\Upsilon$-radical ideals of $B$ containing $I$, we get the proposition. Then

(6.10) Proposition. Let $\mathfrak{a}$ be an ideal of a super real closed ring $A$.

(i) If $\Upsilon_{0} \subseteq \Upsilon$ is a set of generalized root functions, then

$$
\sqrt[\Upsilon]{\mathfrak{a}}=\left\{g \cdot s_{A}(f) \mid g \in A, f \in \mathfrak{a}, f \geq 0, s \in \Upsilon_{0}\right\} .
$$

(ii) If $\Upsilon_{0} \subseteq \Upsilon$ is a set of generalized power functions, then

$$
\sqrt[r]{\mathfrak{a}}=\left\{f \in A \mid s_{A}(|f|) \in \mathfrak{a} \text { for some } s \in \Upsilon_{0}\right\} .
$$

Proof. Follows from (3.3), using the strategy explained in (6.5).

(6.11) Proposition. Let $\varphi: A \rightarrow B$ be a super homomorphism between super real closed rings and let $\mathfrak{b}$ be an ideal of $B$. Then

$$
\sqrt[r]{\varphi^{-1}(\mathfrak{b})}=\varphi^{-1}(\sqrt[\Upsilon]{\mathfrak{b}}) \quad \text { and } \quad \varphi^{-1}(\mathfrak{b})^{\Upsilon}=\varphi^{-1}\left(\mathfrak{b}^{\Upsilon}\right) .
$$

Proof. Follows from (3.5), using the strategy explained in (6.5).

(6.12) TheOREM. Let $A$ be a super real closed ring. Then

$$
\Upsilon \text {-Spec } A:=\{\mathfrak{p} \in \operatorname{Spec} A \mid \mathfrak{p} \text { is } \Upsilon \text {-radical }\}
$$

is a proconstructible subset of $\operatorname{Spec} A$ containing the minimal and the maximal points of $\operatorname{Spec} A$. If $\varphi: A \rightarrow B$ is a super homomorphism, then the restriction $\Upsilon$-Spec $\varphi$ of the map $\operatorname{Spec} \varphi$ to $\Upsilon$-Spec $B$ induces a convex map

$$
\Upsilon \text {-Spec } \varphi: \Upsilon \text {-Spec } B \rightarrow \Upsilon \text {-Spec } A \text {. }
$$

Moreover Spec $\varphi$ has going up if and only if $\Upsilon$-Spec $\varphi$ has going up, and $\operatorname{Spec} \varphi$ has going down if and only if $\Upsilon$-Spec $\varphi$ has going down (see [Tr1, Section 6] for the notions of "convex map", "going up" and "going down").

Proof. From (6.7), (6.10), (6.11) as in the proof of $[\operatorname{Tr} 1,(6.5)]$.

7. Localization of super real closed rings. In this section we want to extend the operation of continuous functions on a given super real closed ring $A$ to certain localizations $S^{-1} A$ of $A$. That is, we want to equip $S^{-1} A$

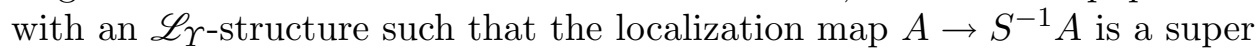
homomorphism. This is not possible for arbitrary multiplicatively closed subsets $S$ of $A$. We need the additional assumption that $t_{A}(f) \in S$ whenever $f \in S$ and $t \in \Upsilon$ (for example, if $f \in A$, then $S=\left\{t_{A}(f) \mid t \in \Upsilon\right\}$ has this property; also complements of $\Upsilon$-radical prime ideals have this property).

In order to reach our goal we first look at the "generic situation" of $n+1$ indeterminate elements $X_{1}, \ldots, X_{n}, Y$ and we must apply a continuous 
function $F \in C\left(\mathbb{R}^{n}\right)$ to the $n$-tuple $\left(X_{1} / Y, \ldots, X_{n} / Y\right)$. In (7.2) below, we show that there are $t \in \Upsilon$ and $G(\bar{x}, y) \in C\left(\mathbb{R}^{n} \times \mathbb{R}\right)$ with

$$
F\left(\frac{x_{1}}{y}, \ldots, \frac{x_{n}}{y}\right)=\frac{G\left(x_{1}, \ldots, x_{n}, y\right)}{t(y)} \quad\left((\bar{x}, y) \in \mathbb{R}^{n} \times(\mathbb{R} \backslash\{0\})\right) .
$$

This equation allows the localization of super real closed rings at multiplicatively closed subsets $S$ of $A$ with $t_{A}(S) \subseteq S(t \in \Upsilon)$.

(7.1) Proposition. Let $\varphi:[0, \infty) \rightarrow(0, \infty)$ be continuous and nondecreasing. Let $s \in \Upsilon$ with $s(x)>0$ for $x>0$. Let $t:(0, \infty) \rightarrow(0, \infty)$ be defined by

$$
t(x):=\frac{s(x)}{\varphi\left(\frac{1}{x \cdot s(x)}\right)}
$$

and let $q:(0, \infty) \times[0, \infty) \rightarrow(0, \infty)$ be defined by

$$
q(x, y):=\varphi\left(\frac{y}{x}\right) \cdot t(x) .
$$

Then $t$ has an extension to a function from $\Upsilon$, and $q$ has a continuous extension $\bar{q}$ on $[0, \infty) \times[0, \infty)$ with $\bar{q}(0, y)=0$ for all $y \geq 0$.

Proof. Since $\varphi$ is nondecreasing we have, for every $x \in(0,1)$,

$$
\frac{1}{\varphi(1)} \leq \frac{1}{x \cdot \varphi(x)} \quad \text { and } \quad \frac{1}{\varphi\left(\frac{1}{x \cdot \varphi(x)}\right)} \leq \frac{1}{\varphi\left(\frac{1}{\varphi(1)}\right)} .
$$

Hence $\lim _{x \rightarrow 0} t(x)=0$, which implies that $t$ has an extension to a function from $\Upsilon$. In order to show that $q$ has a continuous extension onto $[0, \infty) \times$ $[0, \infty)$ we first prove that the function

$$
q_{0}(x, y):=\frac{\varphi\left(\frac{y}{x}\right)}{\varphi\left(\frac{1}{x \cdot s(x)}\right)}
$$

defined on $Q:=(0, \infty) \times[0, \infty)$ is locally bounded in $[0, \infty)^{2}$, i.e. for all $x_{0}, y_{0} \geq 0$ there is an open subset $U$ of $\mathbb{R}^{2}$ containing $\left(x_{0}, y_{0}\right)$ such that $\left.q_{0}\right|_{Q \cap U}$ is bounded. If $x_{0} \neq 0$, then this holds true, since $q_{0}$ is continuous. So let $x_{0}=0$. We take $U:=s^{-1}\left(-\infty, 1 /\left(y_{0}+1\right)\right) \times\left(0, y_{0}+1\right)$. Since $s \in \Upsilon$ and $s(x)>0$ for $x>0$, we have $\left(x_{0}, y_{0}\right)=\left(0, y_{0}\right) \in U$. Pick $(x, y) \in[0, \infty)^{2} \cap U$, hence $x>0$ and $0<y<y_{0}+1<1 / s(x)$. Since $\varphi$ is nondecreasing and positive we get

$$
0<\varphi\left(\frac{y}{x}\right) \leq \varphi\left(\frac{\frac{1}{s(x)}}{x}\right)=\varphi\left(\frac{1}{x \cdot s(x)}\right) .
$$

Thus $0<q_{0}(x, y) \leq 1$ for all $(x, y) \in(0, \infty)^{2} \cap U$ as desired.

Since $q_{0}$ is locally bounded in $[0, \infty)^{2}$ and $s(0)=0$, the function $q(x, y)=$ $q_{0}(x, y) \cdot s(x)$ has a continuous extension $\bar{q}$ on $[0, \infty)^{2}$ with $\bar{q}(0, y)=0$ for all $y \geq 0$. 
The next theorem is the key tool for most of the algebra developed in the rest of the paper:

(7.2) TheOREM. Let $F \in C\left(\mathbb{R}^{n}\right)$. Then there are $t \in \Upsilon$ and a continuous function $G \in C\left(\mathbb{R}^{n} \times \mathbb{R}\right)$ with

$$
F\left(x_{1}, \ldots, x_{n}\right) \cdot t(y)=G\left(x_{1} \cdot y, \ldots, x_{n} \cdot y, y\right) \quad\left((\bar{x}, y) \in \mathbb{R}^{n} \times \mathbb{R}\right) .
$$

More precisely, we can choose $t$ as follows:

(i) If $\varphi:[0, \infty) \rightarrow(0, \infty)$ is continuous, nondecreasing with $|F(\bar{x})| \leq$ $\varphi(|\bar{x}|)$, and $s \in \Upsilon$ with $s(x)>0$ for $x>0$, then we can take

$$
t(x):= \begin{cases}\frac{s(|x|)}{\varphi\left(\frac{1}{|x| \cdot s(|x|)}\right)} & \text { if } x \neq 0, \\ 0 & \text { if } x=0 .\end{cases}
$$

If in addition $s$ is nondecreasing on $(0, \infty)$ with $\lim _{x \rightarrow \infty} s(x)=\infty$, then also $t$ is nondecreasing on $(0, \infty)$ with $\lim _{x \rightarrow \infty} t(x)=\infty$.

(ii) If there is a polynomial $P(T) \in \mathbb{R}[T], T=\left(T_{1}, \ldots, T_{n}\right)$, of total degree d with $|F| \leq|P|$, then for every $s \in \Upsilon$ we can choose $t(y)=$ $y^{d} \cdot s(y)$. In particular, if $F$ is bounded we can choose $t(y)=y$.

Proof. (i) The function $F_{0}(\bar{x}):=F(\bar{x}) / \varphi(|\bar{x}|)$ is bounded. By (7.1), $t \in \Upsilon$ and $t(v) \cdot \varphi(|u|)=q(v \cdot u, v)$ for some continuous function $q \in C\left(\mathbb{R}^{2}\right)$ with $q(u, 0)=0(u \in \mathbb{R})$. Then $F(\bar{x}) \cdot t(y)=F_{0}(\bar{x}) \cdot q(|\bar{x}| \cdot y, y)$. Since $F_{0}$ is bounded and $q(u, 0)=0(u \in \mathbb{R})$, the function $F_{0}\left(x_{1} / y, \ldots, x_{n} / y\right) \cdot q(|\bar{x}|, y)$ can be extended to a continuous function $G \in C\left(\mathbb{R}^{n} \times \mathbb{R}\right)$. So $G\left(x_{1} \cdot y, \ldots\right.$, $\left.x_{n} \cdot y, y\right)=F_{0}(\bar{x}) \cdot q(|\bar{x}| \cdot y, y)=F(\bar{x}) \cdot t(y)$ everywhere.

This shows that $t$ and $G$ have the required property. If $s$ is nondecreasing on $(0, \infty)$ and $\lim _{x \rightarrow \infty} s(x)=\infty$, then it is straightforward to see that $t$ is also nondecreasing on $(0, \infty)$ and $\lim _{x \rightarrow \infty} t(x)=\infty$.

(ii) Let $G_{0} \in C\left(\mathbb{R}^{n} \times(\mathbb{R} \backslash\{0\})\right)$ be defined by

$$
G_{0}\left(x_{1}, \ldots, x_{n}, y\right):=F\left(x_{1} / y, \ldots, x_{n} / y\right) .
$$

Then

$$
\left|G_{0}\left(x_{1}, \ldots, x_{n}, y\right) \cdot y^{d}\right|=\left|F\left(\frac{x_{1}}{y}, \ldots, \frac{x_{n}}{y}\right) \cdot y^{d}\right| \leq\left|P\left(\frac{x_{1}}{y}, \ldots, \frac{x_{n}}{y}\right) \cdot y^{d}\right| .
$$

Since the total degree of $P$ is $d, P\left(x_{1} / y, \ldots, x_{n} / y\right) \cdot y^{d}$ is a polynomial. Hence $G_{0}\left(x_{1}, \ldots, x_{n}, y\right) \cdot y^{d}$ is a continuous function on $\mathbb{R}^{n} \times(\mathbb{R} \backslash\{0\})$ whose absolute value is bounded by a continuous function on $\mathbb{R}^{n} \times \mathbb{R}$. Therefore, and since $s(0)=0, G_{0} \cdot y^{d} \cdot s(y)$ has a continuous extension $G$ on $\mathbb{R}^{n} \times \mathbb{R}$ defined by $G(\bar{x}, y)=0$ if $y=0$.

Clearly $G$ satisfies $F\left(x_{1}, \ldots, x_{n}\right) \cdot y^{d+1}=G\left(x_{1} \cdot y, \ldots, x_{n} \cdot y, y\right)((\bar{x}, y) \in$ $\left.\mathbb{R}^{n} \times \mathbb{R}\right)$. 
(7.3) Definition. Let $A$ be a super real closed ring and let $1 \in S \subseteq A$. We say that $S$ is $\Upsilon$-multiplicatively closed if $S \cdot S \subseteq S$ and $t_{A}(S) \subseteq S$ for all $t \in \Upsilon$.

(7.4) Theorem (Localization of super real closed rings). Let $A$ be a super real closed ring and let $1 \in S \subseteq A$ be $\Upsilon$-multiplicatively closed. Then there is a unique expansion of the localization $S^{-1} A$ to a super real closed ring such that the localization map $A \rightarrow S^{-1} A$ is a super homomorphism. The operation of $F \in C\left(\mathbb{R}^{n}\right)$ on $\left(S^{-1} A\right)^{n}$ is given as follows: Pick $t \in \Upsilon$ and a continuous function $G \in C\left(\mathbb{R}^{n} \times \mathbb{R}\right)$ with

$$
F\left(x_{1}, \ldots, x_{n}\right) \cdot t(y)=G\left(x_{1} \cdot y, \ldots, x_{n} \cdot y, y\right) \quad\left((\bar{x}, y) \in \mathbb{R}^{n} \times \mathbb{R}\right) .
$$

Such functions exist by (7.2). Then for $f_{1}, \ldots, f_{n} \in A$ and $g \in S$,

$$
F_{S^{-1} A}\left(\frac{f_{1}}{g}, \ldots, \frac{f_{n}}{g}\right):=\frac{G_{A}\left(f_{1}, \ldots, f_{n}, g\right)}{t_{A}(g)} .
$$

Proof. First we show that the definition of $F_{S^{-1} A}$ does not depend on the choice of $G$ and $t$. Let $t^{*} \in \Upsilon$ and $G^{*} \in C\left(\mathbb{R}^{n} \times \mathbb{R}\right)$ with

$$
F\left(x_{1}, \ldots, x_{n}\right) \cdot t^{*}(y)=G^{*}\left(x_{1} \cdot y, \ldots, x_{n} \cdot y, y\right) \quad\left((\bar{x}, y) \in \mathbb{R}^{n} \times \mathbb{R}\right) .
$$

Then $t^{*}(y) \cdot G\left(x_{1} \cdot y, \ldots, x_{n} \cdot y, y\right)=t(y) \cdot G^{*}\left(x_{1} \cdot y, \ldots, x_{n} \cdot y, y\right)$ for all $(\bar{x}, y)$ $\in \mathbb{R}^{n} \times \mathbb{R}$. Hence for all $y, z_{1}, \ldots, z_{n} \in \mathbb{R}$ with $y \neq 0$ and $x_{i}:=z_{i} / y$ we have

$$
\begin{aligned}
t^{*}(y) \cdot G\left(z_{1}, \ldots, z_{n}, y\right) & =t^{*}(y) \cdot G\left(x_{1} \cdot y, \ldots, x_{n} \cdot y, y\right) \\
& =t(y) \cdot G^{*}\left(x_{1} \cdot y, \ldots, x_{n} \cdot y, y\right) \\
& =t(y) \cdot G^{*}\left(z_{1}, \ldots, z_{n}, y\right) .
\end{aligned}
$$

If $y=0$, then $t^{*}(y)=t(y)=0$, and this shows that $t^{*}(y) \cdot G\left(z_{1}, \ldots, z_{n}, y\right)=$ $t^{*}(y) \cdot G\left(z_{1}, \ldots, z_{n}, y\right)$ for all $(\bar{z}, y) \in \mathbb{R}^{n} \times \mathbb{R}$. Therefore $t(g) \cdot G^{*}\left(f_{1}, \ldots, f_{n}, g\right)$ $-t^{*}(g) \cdot G\left(f_{1}, \ldots, f_{n}, g\right)=0$, in particular $G\left(f_{1}, \ldots, f_{n}, g\right) / t(g)=G^{*}\left(f_{1}, \ldots\right.$, $\left.f_{n}, g\right) / t^{*}(g)$ in $S^{-1} A$.

Hence we know that $F_{S^{-1} A}$ is well defined and we may consider $S^{-1} A$ as an $\mathscr{L}_{\Upsilon}$-structure with these definitions of the $\underline{F}$ 's.

In order to see that $S^{-1} A$ is a super real closed ring, let $F \in C\left(\mathbb{R}^{n}\right)$ and let $F_{1}, \ldots, F_{n} \in C\left(\mathbb{R}^{k}\right)$. Let $F^{*}:=F \circ\left(F_{1}, \ldots, F_{n}\right) \in C\left(\mathbb{R}^{k}\right)$. Take $t_{1}, \ldots, t_{n} \in \Upsilon$ and $G_{1}, \ldots, G_{n} \in C\left(\mathbb{R}^{k} \times \mathbb{R}\right)$ as in the definition of $F_{1, S^{-1} A}$, $\ldots, F_{n, S^{-1} A}$. For $x_{1}, \ldots, x_{k}, y \in \mathbb{R}$ with $y \neq 0$ we have

$$
\begin{aligned}
F^{*}\left(\frac{x_{1}}{y}, \ldots, \frac{x_{k}}{y}\right) & =F\left(F_{1}\left(\frac{x_{1}}{y}, \ldots, \frac{x_{k}}{y}\right), \ldots, F_{n}\left(\frac{x_{1}}{y}, \ldots, \frac{x_{k}}{y}\right)\right) \\
& =F\left(\frac{G_{1}\left(x_{1}, \ldots, x_{k}, y\right)}{t_{1}(y)}, \ldots, \frac{G_{n}\left(x_{1}, \ldots, x_{k}, y\right)}{t_{n}(y)}\right) \\
& =F\left(\frac{\widetilde{G}_{1}\left(x_{1}, \ldots, x_{k}, y\right)}{\widetilde{t}(y)}, \ldots, \frac{\widetilde{G}_{n}\left(x_{1}, \ldots, x_{k}, y\right)}{\widetilde{t}(y)}\right),
\end{aligned}
$$


where $\tilde{t}:=t_{1} \cdots t_{n} \in \Upsilon$ and

$$
\widetilde{G}_{i}\left(x_{1}, \ldots, x_{k}, y\right)=G_{i}\left(x_{1}, \ldots, x_{k}, y\right) \cdot \prod_{j=1, j \neq i}^{n} t_{j}(y) \in C\left(\mathbb{R}^{n} \times \mathbb{R}\right) .
$$

Take $t \in \Upsilon$ and $G \in C\left(\mathbb{R}^{n} \times \mathbb{R}\right)$ as in the definition of $F_{S^{-1}}$. Then

$$
F^{*}\left(\frac{x_{1}}{y}, \ldots, \frac{x_{k}}{y}\right)=\frac{G\left(\widetilde{G}_{1}\left(x_{1}, \ldots, x_{k}, y\right), \ldots, \widetilde{G}_{n}\left(x_{1}, \ldots, x_{k}, y\right), \widetilde{t}(y)\right)}{t(\widetilde{t}(y))} .
$$

Hence $G^{*}\left(x_{1}, \ldots, x_{k}, y\right):=G\left(\widetilde{G}_{1}\left(x_{1}, \ldots, x_{k}, y\right), \ldots, \widetilde{G}_{n}\left(x_{1}, \ldots, x_{k}, y\right), \widetilde{t}(y)\right)$ $\in C\left(\mathbb{R}^{k} \times \mathbb{R}\right)$ and $t^{*}:=t \circ \tilde{t} \in \Upsilon$ satisfy

$$
F^{*}\left(x_{1}, \ldots, x_{n}\right) \cdot t^{*}(y)=G^{*}\left(x_{1} \cdot y, \ldots, x_{n} \cdot y, y\right) \quad\left((\bar{x}, y) \in \mathbb{R}^{k} \times \mathbb{R}\right) .
$$

This shows that $F_{S^{-1} A}^{*}=F_{S^{-1} A}\left(F_{1, S^{-1} A}, \ldots, F_{n, S^{-1} A}\right)$, so $S^{-1} A$ is a super real closed ring.

Clearly the localization map $A \rightarrow S^{-1} A$ is a super homomorphism with respect to the $\mathscr{L}_{\Upsilon}$-structure provided by the $F_{S^{-1}}$. It remains to show that this structure is uniquely determined by the requirement that the localization map $A \rightarrow S^{-1} A$ is a super homomorphism. Let $B$ be another expansion of $S^{-1} A$ to a super real closed ring such that the localization map $A \rightarrow S^{-1} A$ is a super homomorphism. If $F \in C\left(\mathbb{R}^{n}\right)$, then clearly $F_{B}\left(f_{1}, \ldots, f_{n}\right)=F_{S^{-1} A}\left(f_{1}, \ldots, f_{n}\right)$ for all $f_{1}, \ldots, f_{n} \in A$. If $g \in S$, then take $t \in \Upsilon$ and a continuous function $G \in C\left(\mathbb{R}^{n} \times \mathbb{R}\right)$ with

$$
F\left(x_{1}, \ldots, x_{n}\right) \cdot t(y)=G\left(x_{1} \cdot y, \ldots, x_{n} \cdot y, y\right) \quad\left((\bar{x}, y) \in \mathbb{R}^{n} \times \mathbb{R}\right) .
$$

Since $g$ is a unit in $B$ and $B$ is a super real closed ring we have

$$
F_{B}\left(\frac{f_{1}}{g}, \ldots, \frac{f_{n}}{g}\right) \cdot t_{B}(g)=G_{B}\left(\frac{f_{1}}{g} \cdot g, \ldots, \frac{f_{n}}{g} \cdot g, g\right)=G_{S^{-1} A}\left(f_{1}, \ldots, f_{n}, g\right) \text {. }
$$

Since also $t_{B}(g)=t_{S^{-1} A}(g)$ it follows that

$$
F_{B}\left(\frac{f_{1}}{g}, \ldots, \frac{f_{n}}{g}\right)=\frac{G_{S^{-1} A}\left(f_{1}, \ldots, f_{n}, g\right)}{t_{S^{-1} A}(g)}=F_{S^{-1} A}\left(\frac{f_{1}}{g}, \ldots, \frac{f_{n}}{g}\right)
$$

as desired.

(7.5) Corollary. Let $A$ be a super real closed ring and let $P \subseteq A$ be arbitrary. Let $S$ be the smallest $\Upsilon$-multiplicatively closed subset of $A$ containing $P$. If $\varphi: A \rightarrow B$ is a super homomorphism with $\varphi(P) \subseteq B^{*}$, then also $\varphi(S) \subseteq B^{*}$ and there is a unique super homomorphism $\psi: S^{-1} A \rightarrow B$ such that the diagram

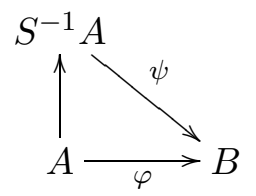

commutes (where $A \rightarrow S^{-1} A$ is the localization map). 
Proof. The set $S$ is the closure of $P$ under multiplication and the application of elements from $\Upsilon$. By (6.12) every maximal ideal of $A$ is $\Upsilon$-radical, hence for every $t \in \Upsilon$ and $b \in B, b$ is a unit in $B$ if and only if $t(b)$ is a unit in $B$. Since $\varphi$ is a super homomorphism, $\varphi(S) \subseteq B^{*}$ and the corollary follows from (7.4).

(7.6) Proposition. Let $A$ be a super real closed domain and let $\mathfrak{p} \in$ $\Upsilon$-Spec $A$ be a proper, direct specialization of (0) in $\Upsilon$-Spec $A$, i.e. there is no $\mathfrak{q} \in \Upsilon$-Spec $A$ with $(0) \subsetneq \mathfrak{q} \subsetneq \mathfrak{p}$. Then $\mathfrak{p}$ is convex in the quotient field of $A$.

Proof. Let $f, g, p \in A, f, g, p>0$ with $f / g<p \in \mathfrak{p}$. We must show that $f / g \in A$. If $g \geq 1$, then $g$ is a unit in $A$ and we are done. So we assume that $g<1$. Since $\mathfrak{p}$ is a proper, direct specialization of $(0)$, there is some strictly increasing $s \in \Upsilon$ with $0<s(p) \leq g$. Since $p<1$ we may replace $s$ by a strictly increasing and bounded function from $\Upsilon$. Since $0<s(p) \leq g$ and $f / g<p$ we have $s(f / g) \leq g$. By (7.4) and since $s$ is bounded, there is $F \in C\left(\mathbb{R}^{2}\right)$ with $s(f / g)=F(f, g) / g$. It follows that $F(f, g) \leq g^{2}$ and the convexity condition for real closed rings implies that $g$ divides $F(f, g)$ in $A$. Thus $s(f / g)=F(f, g) / g \in A$. Since $s \in \Upsilon$ is strictly increasing, there is some $\varepsilon \in \mathbb{R}, \varepsilon>0$, such that $s_{0}:=\left.s\right|_{(-\varepsilon, \varepsilon)}:(-\varepsilon, \varepsilon) \rightarrow s((-\varepsilon, \varepsilon))$ is a homeomorphism. Take $t \in \Upsilon$ with $\left.t\right|_{s((-\varepsilon, \varepsilon))}=s_{0}^{-1}$.

Since $0<f / g<p \in \mathfrak{p}, f / g$ is positive infinitesimal (with respect to $\mathbb{R}$ ) in $\operatorname{qf}(A)$. Then also $s(f / g)$ is positive infinitesimal, hence $0<f / g, s(f / g)<\varepsilon$ and $f / g=t\left(s_{0}(f / g)\right)=t(s(f / g)) \in A$.

\section{Application: o-minimal structures on super real closed fields.} Throughout this section, $T$ is an o-minimal extension of real closed fields in the language $\mathscr{L}$ extending the language of ordered rings. We do not assume that $T$ is complete here. The reference on o-minimality is $[\mathrm{vdD}]$.

(8.1) TheOREM. Let $T$ be an o-minimal (not necessarily complete) theory extending the theory of fields, in the language $\mathscr{L}$ extending the language of ordered rings, and suppose $\mathscr{L}$ has function symbols for all bounded, continuous, T-definable functions. Then

(i) $T$ has quantifier elimination.

(ii) Let $M \models T$ and let $A$ be an $\mathscr{L}$-substructure of $M$. Then $A$ is a domain and the quotient field of $A$ in $M$ is an elementary substructure of $M$.

This is a folklore theorem in the case when $T$ is complete. I am not aware of a reference and I will give the proof for arbitrary $T$.

First the explanation of the term " $T$-definable function". 
(8.2) Definition. Let $T$ be an $\mathscr{L}$-theory. A $T$-definable function is an $\mathscr{L}$-formula $\varphi\left(x_{1}, \ldots, x_{n}, y\right)$ for some $n \in \mathbb{N}_{0}$ such that

$$
T \vdash \forall \bar{x} \exists ! y \varphi(\bar{x}, y) .
$$

In other words, $\varphi(\bar{x}, y)$ is a $T$-definable function if and only if for every model $M$ of $T, \varphi(\bar{x}, y)$ defines the graph of a function $M^{\bar{x}} \rightarrow M$.

If $M$ is a model of $T$ and $f: M^{n} \rightarrow M$ is a map, then $f$ is called $T$-definable if the graph of $f$ is defined by a $T$-definable function. Now let $T$ be an extension of an ordered abelian group. A $T$-definable function $\varphi(\bar{x}, y)$ is continuous if

$$
\begin{aligned}
& T \vdash \forall \bar{x}, y \forall \varepsilon>0 \exists \delta>0 \forall \bar{u}, v \\
& \varphi(\bar{x}, y) \wedge \varphi(\bar{u}, v) \wedge \max _{i}\left\{\left|x_{i}-u_{i}\right|\right\}<\delta \rightarrow|y-v|<\varepsilon .
\end{aligned}
$$

Again, a formula $\varphi(\bar{x}, y)$ is a continuous $T$-definable function if $\varphi$ defines a continuous function $M^{\bar{x}} \rightarrow M$ in every model $M$ of $T$.

(8.3) Definition. Let $T$ be o-minimal (not necessarily complete) in the language $\mathscr{L}$ expanding the language of ordered rings. We say that $\mathscr{L}$ has function symbols for all (bounded) continuous, T-definable functions if for every $T$-definable, continuous function $\varphi(\bar{x}, y)$ (such that there is some $n \in \mathbb{N}$ with $T \vdash \forall \bar{x}, y \varphi(\bar{x}, y) \rightarrow|y| \leq n)$ there is a function symbol $f(\bar{x})$ in $\mathscr{L}$ with $T \vdash \forall \bar{x}, y \varphi(\bar{x}, y) \leftrightarrow y=f(\bar{x})$.

For each $\mathscr{L}$-formula $\varphi(\bar{x}), \bar{x}=\left(x_{1}, \ldots, x_{n}\right)$, let $D_{\varphi}(\bar{z}, y)$ be the $\mathscr{L}$-formula

$$
y=\inf \{\|\bar{x}-\bar{z}\| \mid \varphi(\bar{x}) \text { holds }\} .
$$

So if $M$ is a model of $T$, then $D_{\varphi}(\bar{z}, y)$ is the graph of the distance function $d_{\varphi}(\bar{z})$ of the set of all realizations of $\varphi(\bar{x})$.

Proof of Theorem (8.1)(i). If $\varphi(\bar{x})$ is an $\mathscr{L}$-formula defining a closed set in every model of $T$, then there is a $T$-definable bounded continuous function which defines the graph of $d_{\varphi}(\bar{x}) /\left(1+d_{\varphi}(\bar{x})^{2}\right)$ in every model of $T$. Since the zero set of this function is the set of realizations of $\varphi$ in every model of $T$, it remains to show that every $\mathscr{L}$-formula is $T$-equivalent to a boolean combination of formulas, each defining a closed set in every model of $T$.

In order to prove this we use the following facts from o-minimality theory. Let $M$ be a model of $T$ and let $X \subseteq M^{n}$ be definable. Then

1. The frontier $\partial X:=\bar{X} \backslash X$ of $X$ is of dimension strictly less than $\operatorname{dim} X$ (cf. $[\mathrm{vdD}, \mathrm{IV},(1.8)])$. We define $\partial_{0} X=X, \partial_{n+1}(X):=\partial\left(\partial_{n}(X)\right)$.

2. Let $L(X):=\left\{x \in X \mid\right.$ there is $\varepsilon \in M, \varepsilon>0$, such that $B_{\varepsilon}(x) \cap X$ is closed in $\left.B_{\varepsilon}(x)\right\}$, where $B_{\varepsilon}(x)$ is the open ball of radius $\varepsilon$ around $x$. Then $L(X)$ is a locally closed subset of $M^{n}$ which is dense and open in $X$. Moreover $X \backslash L(X)=\partial_{2}(X)$; this is straightforward from the 
observation that $B_{\varepsilon}(x) \cap X$ is closed in $B_{\varepsilon}(x)$ if and only if $B_{\varepsilon}(x) \cap$ $\partial X=\emptyset$.

It follows that

$$
X=L(X) \cup L\left(\partial_{2}(X)\right) \cup L\left(\partial_{4}(X)\right) \cup \cdots \cup L\left(\partial_{2 k}(X)\right),
$$

where $k \in \mathbb{N}$ is such that $2 k \geq n$ : by item $1, \partial_{2 k}(X)$ is a finite set, hence the process stops at this index. We shall now formulate $(*)$ in terms of formulas.

For each $\mathscr{L}$-formula $\varphi(\bar{x}), \bar{x}=\left(x_{1}, \ldots, x_{n}\right)$, let $\psi_{\varphi}(\bar{x})$ be the formula which defines the closure $\varphi\left[M^{n}\right]$ in models $M$ of $T$. Let $O_{\varphi}(\bar{z})$ be the $\mathscr{L}$ formula

$$
\exists \bar{x}, \varepsilon\|\bar{x}-\bar{z}\|<\varepsilon \wedge \varphi(\bar{x}) \wedge \text { " } B_{\varepsilon}(\bar{x}) \cap \varphi(\bar{x}) \text { is closed in } B_{\varepsilon}(\bar{x}) " .
$$

Hence in every model $M$ of $T, O_{\varphi}(\bar{z})$ defines an open subset of $M^{n}$ such that $L\left(\varphi\left[M^{n}\right]\right)=O_{\varphi}\left[M^{n}\right] \cap \varphi\left[M^{n}\right]$. But then also $L\left(\varphi\left[M^{n}\right]\right)=O_{\varphi}\left[M^{n}\right] \cap \psi_{\varphi}\left[M^{n}\right]$.

Pick $k \in \mathbb{N}$ with $2 k \geq n$. We define formulas $\varphi_{0}(\bar{x}), \psi_{0}(\bar{x}), \chi_{0}(\bar{x}), \ldots$, $\varphi_{k}(\bar{x}), \psi_{k}(\bar{x}), \chi_{k}(\bar{x})$ as follows: $\varphi_{0}:=\varphi, \psi_{0}:=\psi_{\varphi}, \chi_{0}:=\neg O_{\varphi}$ and inductively,

$$
\begin{aligned}
& \varphi_{i+1}=\varphi_{i} \wedge \neg\left(\left(\psi_{0} \wedge \neg \chi_{0}\right) \vee \cdots \vee\left(\psi_{i} \wedge \neg \chi_{i}\right)\right), \\
& \psi_{i+1}=\psi_{\varphi_{i+1}}, \quad \chi_{i+1}=\neg O_{\varphi_{i+1}} .
\end{aligned}
$$

Inductively we see that $\left(\psi_{i} \wedge \neg \chi_{i}\right)\left[M^{n}\right]$ defines $L\left(\partial_{2 i}\left(\varphi\left[M^{n}\right]\right)\right)$ and $\varphi_{i}$ defines $\partial_{2 i}\left(\varphi\left[M^{n}\right]\right)$ in models $M$ of $T$. Since all the $\psi_{i}$ and $\chi_{i}$ define closed sets in models of $T$, the representation $(*)$ shows:

(8.4) Proposition.

$$
T \vdash \forall \bar{x}\left[\varphi(\bar{x}) \leftrightarrow \bigvee_{i=0}^{k} \psi_{i}(\bar{x}) \wedge \neg \chi_{i}(\bar{x})\right]
$$

and for every model $M$ of $T$ the sets $\psi_{i}\left[M^{n}\right]$ and $\chi_{i}\left[M^{n}\right]$ are closed subsets of $M^{n}$.

In particular $T$ has quantifier elimination.

Proof of Theorem 8.1(ii). Of course, $A$ is a domain. In order to prove that the quotient field of $A$ is an elementary substructure of $M$ it is enough to show that $\operatorname{qf}(A)$ is the definable closure of $A$ in $M$ (by o-minimality). Take a function $F: M^{n} \rightarrow M$, 0-definable in $M$, and let $a_{1}, \ldots, a_{n} \in M$. We must show that $F\left(a_{1}, \ldots, a_{n}\right) \in \operatorname{qf}(A)$. Clearly, we may assume $\left|F\left(a_{1}, \ldots, a_{n}\right)\right|$ $\leq 1$ and that $\operatorname{dim}\left\{a_{1}, \ldots, a_{n}\right\}=n$, where dim denotes the dimension in the sense of $\operatorname{Th}(M)$. We may also assume that $F$ is $T$-definable and less than or equal to 1 everywhere. Suppose the graph of $F$ is defined by $\gamma_{0}(\bar{x}, y)$ in $M$; then the formula

$\gamma(\bar{x}, y):=\left[\gamma_{0}(\bar{x}, y) \wedge \exists ! z|z| \leq 1 \wedge \gamma_{0}(\bar{x}, z)\right] \vee\left[y=0 \wedge \neg \exists ! z|z| \leq 1 \wedge \gamma_{0}(\bar{x}, z)\right]$ 
defines the graph of $F$ and defines a function of absolute value $\leq 1$ in every $\mathscr{L}$-structure.

We have to find $T$-definable, bounded, continuous functions $\varphi(\bar{x}, y)$, $\psi(\bar{x}, y)$ such that for every model $M$ of $T$ we have $M \models F(\bar{a}) \cdot H(\bar{a})=$ $G(\bar{a}) \wedge H(\bar{a}) \neq 0$, where $G, H: M^{n} \rightarrow M$ denote the functions defined by $\varphi, \psi$ in $M$ respectively. Let $\delta(\bar{x})$ be the formula which says that $F$ is continuous at $\bar{x}$ and of absolute value $<2$ :

$$
\delta(\bar{x}):=|F(\bar{x})|<2 \wedge \forall \varepsilon>0 \exists \delta>0 \forall \bar{y}\|\bar{x}-\bar{y}\|<\delta \rightarrow|F(\bar{x})-F(\bar{y})|<\varepsilon .
$$

Let $\psi(\bar{x}, y)$ be the formula which defines the distance function to the complement of $\delta(\bar{x})$, composed with the function $y_{0}^{2} /\left(1+y_{0}^{2}\right)$ if this complement is nonempty, and the constant function 1 otherwise. Hence

$$
\begin{aligned}
& \psi(\bar{x}, y)=[\forall \bar{u} \delta(\bar{u}) \wedge y=1] \\
& \vee\left[\exists \bar{u}, y_{0} \neg \delta(\bar{u}) \wedge y=\frac{y_{0}^{2}}{y_{0}^{2}+1} \wedge y_{0}=\inf \{z \mid \exists \bar{u} \neg \delta(\bar{u}) \wedge\|\bar{x}-\bar{u}\|=z\}\right] .
\end{aligned}
$$

Clearly $\psi(\bar{x}, y)$ is a bounded, continuous, $T$-definable function. Finally, let

$$
\varphi(\bar{x}, y):=\exists y_{1}, y_{2} y=y_{1} \cdot y_{2} \wedge \gamma\left(\bar{x}, y_{1}\right) \wedge \psi\left(\bar{x}, y_{2}\right)
$$

We claim that $\varphi(\bar{x}, y)$ is a bounded, continuous, T-definable function. To see this, let $N$ be a model of $T$ and let $f, h$ be the functions defined by $\gamma(\bar{x}, y), \psi(\bar{x}, y)$ in $N$ respectively. By definition, $\varphi(\bar{x}, y)$ defines the graph of the product $g:=f \cdot h$ in $N$ and we must show that $g$ is continuous at every $\bar{c} \in N^{\bar{x}}$. The formula $\delta(\bar{x})$ defines the set

$$
S:=\left\{\bar{n} \in N^{\bar{x}} \mid f \text { is continuous at } \bar{n} \text { and }|f(\bar{n})|<2\right\} .
$$

If $S=N^{\bar{x}}$, then by definition $h=1$ and $g=f$ is continuous of absolute value $\leq 1$. So we assume that $S \neq N^{\bar{x}}$. Then $h$ is the distance function to $N^{\bar{x}} \backslash S$ composed with the function $y_{0}^{2} /\left(1+y_{0}^{2}\right)$, and by definition of $S$ we have $|f| \leq 2$ on $N^{\bar{x}}$.

If $\bar{c} \in S$, then $f$ is continuous at $\bar{c}$, so $g=f \cdot h$ is also continuous at $\bar{c}$.

If $\bar{c} \notin S$, then $h(\bar{c})=0$ and $|g(\bar{x})| \leq 2 h(\bar{x}) \rightarrow 0$ as $\bar{x} \rightarrow \bar{c}$. This shows that $g$ is also continuous at $\bar{c}$.

So we have defined bounded (by $2 \in \mathbb{N}$ ), continuous, $T$-definable functions $\varphi, \psi$ and we return to our initial data, $M, A$ and $\bar{a} \in A^{\bar{x}}$. Let $G, H: M^{n} \rightarrow M$ denote the functions defined by $\varphi, \psi$ in $M$ respectively. We have assumed that $|F(\bar{a})| \leq 1$ and that $\operatorname{dim}\left\{a_{1}, \ldots, a_{n}\right\}=n$. Let $\mathscr{P}$ be the definable closure of $\emptyset$ in $M$. By o-minimality, there is an open, 0-definable subset $U$ of $\mathscr{P}^{n}$ such that $F$ is continuous and of absolute value $<2$ in $U$, and $\bar{a} \in U_{M}$. Therefore, the function $H$ defined by $\psi(\bar{x}, y)$ in $M$ is not zero at $\bar{a}$. It follows that $M=F(\bar{a}) \cdot H(\bar{a})=G(\bar{a}) \wedge H(\bar{a}) \neq 0$, as desired. 
(8.5) Theorem. Let $A$ be a super real closed ring and let $\mathfrak{p}$ be an $\Upsilon$ radical prime ideal of $A$.

(i) There is a unique expansion $\mathcal{K}$ of the quotient field of $A$ at $\mathfrak{p}$ such that the residue map $A \rightarrow \mathcal{K}$ is a super homomorphism.

(ii) For every o-minimal expansion $M$ of the field $\mathbb{R}$ in the language $\mathscr{L}$ (consisting of function symbols for continuous definable functions $\left.\mathbb{R}^{n} \rightarrow \mathbb{R}\right)$, the restriction of $\mathcal{K}$ to $\mathscr{L}$ is an elementary extension of $M$.

Proof. (i) holds by (6.3) and (7.4).

(ii) Let $N$ be the restriction of $\mathcal{K}$ to $\mathscr{L}$. Since the super real closed field $\mathbb{R}$ is existentially closed in $\mathcal{K}$ by (5.6), $M$ is also existentially closed in $N$. Hence there is an $\mathscr{L}$-embedding of $N$ into an elementary extension $M^{\prime}$ of $M$. By (8.1)(ii) applied to the theory of $M, N$ is a model of that theory. From (8.1)(i) it follows that $M$ is an elementary substructure of $N$.

9. Convexity in super real closed rings. If $F: \mathbb{R}^{n} \rightarrow \mathbb{R}$ is continuous with $F(0)=0$, then there is some $s \in \Upsilon$ with

$$
\left|F\left(x_{1}, \ldots, x_{n}\right)\right| \leq s\left(x_{1}\right)+\cdots+s\left(x_{n}\right) \quad\left(x_{i} \in \mathbb{R}\right) .
$$

For example the map $s(t):=|t| \vee \max \left\{|F(\bar{x})||| x_{1}|, \ldots,| x_{n}|\leq| t \mid\right\}$ has this property. This observation generates the following super real closed rings:

(9.1) Proposition. Let $B$ be a super real closed ring and let $M$ be a subgroup of $(B,+)$ such that

1. $M$ is absolutely convex in $B$, i.e. for all $b, m \in B$ we have

$$
|b| \leq|m|, m \in M \Rightarrow b \in M .
$$

2. $M$ is closed under $\Upsilon$, i.e. $s_{B}(M) \subseteq M(s \in \Upsilon)$. Then

(i) If $A \subseteq B$ is a super real closed subring of $B$ and $M$ is an $A$ submodule of $B$, then $A+M$ is a super real closed subring of $B$.

(ii) The set $C:=\{c \in B \mid c \cdot M \subseteq M\}$ is an absolutely convex super real closed subring of $B$, and $M$ is an ideal of $C$.

Proof. (i) Let $F \in C\left(\mathbb{R}^{n}\right)$, let $\bar{a} \in A^{n}$ and let $\bar{\mu} \in M^{n}$. By assumption we know that $F_{B}(\bar{a}) \in A$ and it is enough to show that $F_{B}(\bar{a}+\bar{\mu})-F_{B}(\bar{a}) \in M$.

Let $G(\bar{x}, \bar{y}):=F(\bar{x}+\bar{y})-F(\bar{x})$. Hence $G: \mathbb{R}^{n} \times \mathbb{R}^{n} \rightarrow \mathbb{R}$ is continuous. Let $H: \mathbb{R}^{n} \times \mathbb{R}^{n} \rightarrow \mathbb{R}$ be defined by $H(\bar{x}, \bar{y})=y_{1}^{2}+\cdots+y_{n}^{2}$. Then every zero of $H$ is a zero of $G$. Since $\mathbb{R}^{n}$ has computable $z$-radicals, there is a homeomorphism $s \in \Upsilon$ and some $Q \in C\left(\mathbb{R}^{n} \times \mathbb{R}^{n}\right)$ such that $s \circ|G|=Q \cdot H$. Take $\widetilde{s} \in \Upsilon$ such that $\left|Q\left(t_{1}, \ldots, t_{2 n}\right)\right| \leq|Q(\overline{0})|+\widetilde{s}\left(t_{1}\right)+\cdots+\widetilde{s}\left(t_{2 n}\right)$ on $\mathbb{R}^{2 n}$. 
Then

$\left|s_{B} \circ\right| G_{B}(\bar{a}, \bar{\mu})||$

$\leq\left(\mu_{1}^{2}+\cdots+\mu_{n}^{2}\right) \cdot\left(|Q(\overline{0})|+\widetilde{s}_{B}\left(a_{1}\right)+\cdots+\widetilde{s}_{B}\left(a_{n}\right)+\widetilde{s}_{B}\left(\mu_{1}\right)+\cdots+\widetilde{s}_{B}\left(\mu_{n}\right)\right)$.

By assumption 2 and since $M$ is an $A$-module and $A$ is closed under $\Upsilon$, the right hand side of this inequality is in $M$. By assumption 1, we get $s_{B} \circ\left|G_{B}(\bar{a}, \bar{\mu})\right| \in M$. Since $s$ is a homeomorphism, assumption 2 implies $\left|G_{B}(\bar{a}, \bar{\mu})\right| \in M$, hence by assumption 1 again, $G_{B}(\bar{a}, \bar{\mu}) \in M$, as desired.

(ii) Clearly $C$ is a subring of $B$, and $M$ is a $C$-module. By assumptions 1 and $2, M \subseteq C$, hence $M$ is an ideal of $C$. If $c \in C$ and $b \in B$ with $|b| \leq|c|$, then $b \cdot M \subseteq M$, since for $m \in M$ we have $|b \cdot m| \leq|c \cdot m|$ and $c \cdot m \in M$; so by assumption $1, b \cdot m \in M$.

Hence $C$ is an absolutely convex subring of $B$ and it remains to show that $C$ is a super real closed subring of $B$. Since $C$ is an absolutely convex subring and every $F \in C\left(\mathbb{R}^{n}\right)$ can be bounded above by a natural number plus the sum of $s\left(x_{i}\right)(1 \leq i \leq n)$ for some $s \in \Upsilon$, it is enough to show that $C$ is closed under $\Upsilon$.

Let $s \in \Upsilon$. Since $C$ is absolutely convex and there is an increasing homeomorphism $h \in \Upsilon$ such that $|s| \leq|h|$ we may assume that $s$ is an increasing homeomorphism $\mathbb{R} \rightarrow \mathbb{R}$. By (7.2) (i), there is some $t \in \Upsilon$ with $\lim _{x \rightarrow \infty} t(x)=\infty$ and some continuous $G: \mathbb{R}^{2} \rightarrow \mathbb{R}$ such that $s(x) \cdot t(y)=$ $G(x \cdot y, y)$. As $\lim _{x \rightarrow \infty} t(x)=\infty$, there is some $\widetilde{t} \in \Upsilon$ such that $x \leq t \circ \widetilde{t}(x)$ for all $x \geq 0$. Take $c \in C$ and $m \in M$. Then

$$
\left|s_{B}(c) \cdot m\right| \leq\left|s_{B}(c) \cdot t_{B}\left(\widetilde{t}_{B}(|m|)\right)\right|=G_{B}\left(c \cdot \widetilde{t}_{B}(|m|), \widetilde{t}_{B}(|m|)\right) .
$$

Since $\widetilde{t}_{B}(|m|) \in M$ by assumption 2 and $c \in C$ we know that also $c \cdot \widetilde{t}_{B}(|m|)$ $\in M$. Since $|G(x, y)| \leq s_{1}(x)+s_{2}(y)$ for some $s_{1}, s_{2} \in \Upsilon$ (observe that $G(0,0)=0)$ we get $\left|s_{B}(c) \cdot m\right| \leq s_{1, B}\left(c \cdot \widetilde{t}_{B}(|m|)\right)+s_{2, B}\left(\widetilde{t}_{B}(|m|)\right) \in M$. Вy assumption 1 we get $s_{B}(c) \cdot m \in M$.

This shows that $s_{B}(c) \in C$.

(9.2) Corollary. Let $B$ be a super real closed ring and let $A$ be a subring of $B$, closed under $\Upsilon$. Then

(i) The convex hull of $A$ in $B$ (defined as $\{b \in B|| b|\leq| a \mid$ for some $a \in A\})$ is a super real closed subring of $B$.

(ii) If $A$ is a super real closed subring of $B$ and $I$ is an $\Upsilon$-radical ideal of $B$, then $A+I$ is a super real closed subring of $B$.

(iii) If $A$ is an $\Upsilon$-subring of $B$, and $I$ is an $\Upsilon$-radical ideal of $A$, then $C:=\{b \in B \mid b \cdot J \subseteq J\}$ with $J:=\{b \in B|| b|\leq| a \mid$ for some $a \in I\}$ is a convex super real closed subring of $B$, and $J$ is an $\Upsilon$-radical ideal of $C$, lying over $I$. 
Proof. (i) holds, since the convex hull $M$ of $A$ in $B$ satisfies conditions 1 and 2 of (9.1). Now apply (9.1) for $M$ and the super real closed subring $\mathbb{R}$ of $B$.

(ii) Let $M:=I$. Since $M$ is a radical ideal of $B$, it satisfies condition 1 of (9.1). Since $M$ is $\Upsilon$-radical, it also satisfies condition 2 of (9.1). Hence $A+I=A+M$ is a super real closed subring of $B$.

(iii) By (9.1)(ii) it is enough to show that $J$ satisfies conditions 1 and 2 of (9.1). By definition, $J$ satisfies condition 1 of (9.1) and it remains to show that $J$ is closed under $\Upsilon$. Let $s \in \Upsilon$. Take some strictly increasing homeomorphism $t \in \Upsilon$ with $|s| \leq|t|$. Then for $b \in J$ and $a \in I$ with $|b| \leq|a|$ we have $\left|s_{B}(b)\right| \leq\left|t_{B}(b)\right| \leq\left|t_{B}(a)\right|$. Since $t_{B}(a) \in I$ we get $s_{B}(b) \in J$ as desired.

10. An extended Gelfand-Kolmogorov theorem. Let $A$ be a ring with normal spectrum, i.e. every prime ideal of $A$ is contained in a unique maximal ideal of $A$. Let $r: \operatorname{Spec} A \rightarrow(\operatorname{Spec} A)^{\max }$ be the map which sends a prime ideal to the maximal ideal containing it. Then $r$ is continuous and any ring homomorphism $\varphi: A \rightarrow B$ induces a continuous map $\iota:(\operatorname{Spec} B)^{\max } \rightarrow$ $(\operatorname{Spec} A)^{\max }$ by mapping $\mathfrak{m}$ to $r\left(\varphi^{-1}(\mathfrak{m})\right.$ ) (in the proof of $(10.1)$ below, this is explained with references to proofs).

The Gelfand-Kolmogorov theorem says that this map is a homeomorphism if $A=C^{*}(X), B=C(X)$ and $\varphi$ is the inclusion.

We generalize this in (10.1) to arbitrary rings with normal spectrum by calculating the fibres of $\iota$. In (10.5) we apply (10.1) to convex subrings of real closed rings (which then gives back the original statement). At the end of this section we apply our results to rings of continuous functions. In the next section we apply the results to super real closed rings.

First some notations. For a subset $S$ of an arbitrary ring $A, V(S)$ denotes the set of prime ideals of $A$ containing $S$. Recall from [Tr1, Section 4], the ideal construction $O(\mathfrak{a})$ for an ideal $\mathfrak{a}$ of a $\operatorname{ring} A . O(\mathfrak{a})$ is the radical ideal which defines the Zariski closure of $\bigcap_{V(\mathfrak{a}) \subseteq O, O \text { open }} O$ in $\operatorname{Spec} A$. By $[\operatorname{Tr} 1,(4.9)]$,

$$
O(\mathfrak{a})=\left\{f \in A \mid \text { there are } a \in \mathfrak{a} \text { and } k \in \mathbb{N} \text { such that } f^{k} \cdot(1-a)=0\right\}
$$

is the radical ideal generated by the kernel of the localization map $A \rightarrow$ $(1+\mathfrak{a})^{-1} A$.

(10.1) ThEOREM (Gelfand-Kolmogorov for rings with normal spectrum). Let $A$ be a ring with normal spectrum and let $\varphi: A \rightarrow B$ be a ring homomorphism. Then the map

$$
\iota:(\operatorname{Spec} B)^{\max } \rightarrow(\operatorname{Spec} A)^{\max }, \quad \mathfrak{n} \mapsto \sqrt[\max ]{\varphi^{-1}(\mathfrak{n})},
$$

is continuous and closed with image $V(\operatorname{Ker} \varphi)^{\max }$, and for each $\mathfrak{m}$ in 
$(\operatorname{Spec} A)^{\max }$ the fibre of $\iota$ at $\mathfrak{m}$ is

$$
\iota^{-1}(\mathfrak{m})=V(O(\mathfrak{m}) \cdot B)^{\max } .
$$

Moreover the following are equivalent:

(i) $\iota$ is injective.

(ii) For every maximal ideal $\mathfrak{m}$ of $A$ the localization $B_{\mathfrak{m}}$ of the A-algebra $B$ at $\mathfrak{m}$ is a local ring.

(iii) For all $f \in B$ the ideals $\varphi^{-1}(f B)$ and $\varphi^{-1}((1-f) B)$ of $A$ are coprime.

Proof. $\iota$ is continuous, since $\iota$ is the composition of the continuous map $\operatorname{Spec} \varphi$ with the retraction $r: \operatorname{Spec} A \rightarrow(\operatorname{Spec} A)^{\max }$. That $r$ is continuous (and closed) can be found in [Kn-Sch, Kapitel III, §6, Satz 5], under the additional assumption that $\operatorname{Spec} A$ is completely normal-but this proof only needs the assumption that every prime ideal of $A$ is contained in a unique maximal ideal.

So we have a continuous map $\iota:(\operatorname{Spec} B)^{\max } \rightarrow(\operatorname{Spec} A)^{\max }$. Since $(\operatorname{Spec} B)^{\max }$ is compact and $(\operatorname{Spec} A)^{\max }$ is Hausdorff (this follows again from the assumption that every prime ideal of $A$ is contained in a unique maximal ideal, together with the separation lemma for spectral spaces, cf. $[\operatorname{Tr} 1,(2.6)])$, we deduce that $\iota$ is closed.

We now prove that $\iota^{-1}(\mathfrak{m})=V(O(\mathfrak{m}) \cdot B)^{\max }$. Let $\mathfrak{m} \in(\operatorname{Spec} A)^{\max }$ and $\mathfrak{n} \in(\operatorname{Spec} B)^{\max }$.

$\subseteq$ : If $\iota(\mathfrak{n})=\mathfrak{m}$, then $O(\mathfrak{m}) \subseteq \varphi^{-1}(\mathfrak{n})$, by definition of $O(\mathfrak{m})$. Consequently, $\mathfrak{n} \in V(O(\mathfrak{m}) \cdot B)^{\max }$.

$\supseteq$ : If $O(\mathfrak{m}) \cdot B \subseteq \mathfrak{n}$, then $O(\mathfrak{m}) \subseteq \varphi^{-1}(\mathfrak{n})$, and since $\operatorname{Spec} A$ is normal we get $\iota(\mathfrak{n})=\mathfrak{m}$.

Clearly, the image of $\iota$ is contained in $V(\operatorname{Ker} \varphi)^{\max }$. Conversely, if $\mathfrak{m}$ in $(\operatorname{Spec} A)^{\max }$ is not in the image of $\iota$, then we already know that $1 \in O(\mathfrak{m}) \cdot B$; so take $a_{1}, \ldots, a_{n} \in O(\mathfrak{m})$ and $b_{1}, \ldots, b_{n} \in B$ with

$$
1=\varphi\left(a_{1}\right) b_{1}+\cdots+\varphi\left(a_{n}\right) b_{n} .
$$

Since $a_{i} \in O(\mathfrak{m})$ there are $\mu_{1}, \ldots, \mu_{n} \in \mathfrak{m}$ and some $k \in \mathbb{N}$ with $a_{i}^{k} \cdot\left(1+\mu_{i}\right)$ $=0$. By taking the $(k n)$ th power of $(*)$ we may assume that $k=1$. Take $a:=\left(1+\mu_{1}\right) \cdots\left(1+\mu_{n}\right)$. Then $a \notin \mathfrak{m}$ and from $(*)$ we get $\varphi(a)=0$. This shows that the image of $\iota$ is $V(\operatorname{Ker} \varphi)^{\max }$.

It remains to prove the characterizations of the injectivity of $\iota$.

(i) is equivalent to (ii), since for every $\mathfrak{m} \in(\operatorname{Spec} A)^{\max }$ the natural map Spec $B_{\mathfrak{m}} \rightarrow \operatorname{Spec} B$ is a homeomorphism onto the set of all prime ideals $\mathfrak{q}$ of $B$ with $\varphi^{-1}(\mathfrak{q}) \subseteq \mathfrak{m}$.

(i) $\Rightarrow$ (iii). Suppose $\iota$ is injective and let $f \in B$. Suppose there is some maximal ideal $\mathfrak{m}$ of $A$ containing $\varphi^{-1}(f B)+\varphi^{-1}((1-f) B)$. Let $\mathfrak{b}:=f \cdot B$. We apply what we have already proved to the $\left(A / \varphi^{-1}(\mathfrak{b})\right)$-algebra $B / \mathfrak{b}$. This 
shows that there is some $\mathfrak{n}_{1} \in(\operatorname{Spec} B)^{\max }$ containing $f$ with $\iota\left(\mathfrak{n}_{1}\right)=\mathfrak{m}$. The same argument applied to $1-f$ shows that there is some $\mathfrak{n}_{2} \in(\operatorname{Spec} B)^{\max }$ containing $1-f$ with $\iota\left(\mathfrak{n}_{2}\right)=\mathfrak{m}$. Since $\iota$ is injective we must have $\mathfrak{n}_{1}=\mathfrak{n}_{2}$, which is impossible, as $f \in \mathfrak{n}_{1}$ and $1-f \in \mathfrak{n}_{2}$.

(iii) $\Rightarrow\left(\right.$ i). Suppose $1 \in \varphi^{-1}(f B)+\varphi^{-1}((1-f) B)$ for all $f \in B$. Let $\mathfrak{n}_{1}, \mathfrak{n}_{2} \in(\operatorname{Spec} B)^{\max }$ with $\mathfrak{n}_{1} \neq \mathfrak{n}_{2}$. Then there are $f \in \mathfrak{n}_{1}$ and $g \in \mathfrak{n}_{2}$ with $f+g=1$. By assumption, there are $a_{f} \in \varphi^{-1}\left(\mathfrak{n}_{1}\right)$ and $a_{g} \in \varphi^{-1}\left(\mathfrak{n}_{1}\right)$ with $1=a_{f}+a_{g}$. So $\iota\left(\mathfrak{n}_{1}\right) \neq \iota\left(\mathfrak{n}_{2}\right)$. This shows that $\iota$ is injective.

(10.2) Corollary. Let $A$ be a ring with normal spectrum and let $\varphi$ : $A \rightarrow B$ be an $A$-algebra. Let

$$
\iota:(\operatorname{Spec} B)^{\max } \rightarrow(\operatorname{Spec} A)^{\max }, \quad \mathfrak{n} \mapsto \sqrt[\max ]{\varphi^{-1}(\mathfrak{n})},
$$

and let $\mathfrak{b}$ be an ideal of $B$. Then the restriction $r$ of $\iota$ to $V(\mathfrak{b})^{\max }$ is a surjective, continuous and closed map

$$
r: V(\mathfrak{b})^{\max } \rightarrow V\left(\varphi^{-1}(\mathfrak{b})\right)^{\max } .
$$

If $\iota$ is injective, then $r$ is a homeomorphism.

Proof. Let $r$ denote the restriction of $\iota$ to $V(\mathfrak{b})^{\max }$. Clearly $r$ has values in $V\left(\varphi^{-1}(\mathfrak{b})\right)^{\text {max }}$. Since $A / \varphi^{-1}(\mathfrak{b})$ is again normal we may apply (10.1) to the injective ring homomorphism $A / \varphi^{-1}(\mathfrak{b}) \rightarrow B / \mathfrak{b}$ and we see that $r$ is onto $V\left(\varphi^{-1}(\mathfrak{b})\right)^{\max }$. If $\iota$ is injective, then $r$ is a continuous bijection from a quasi-compact space onto a Hausdorff space, hence a homeomorphism.

Our main application of (10.1) will concern the case where $B$ is a real closed ring and $A$ is squeezed between $B$ and the holomorphy ring of $B$. Recall that the holomorphy ring $\operatorname{Hol}(B)$ of a real closed ring $B$ is the subring of all elements $f \in B$ such that $|f| \leq N$ for some $N \in \mathbb{N}$. As a preparation we need

(10.3) Lemma. Let $A$ be a real closed ring. Then for all $f \in A, f^{2}+$ $(1-f)^{2}$ is a unit in $A$ with $f^{2}+(1-f)^{2} \geq 1 / 2$.

Proof. We may consider $A$ as a ring of functions $X \rightarrow R$ for some real closed field $R$ and some set $X$.

Let $\varphi: \mathbb{R}_{\text {alg }} \rightarrow \mathbb{R}_{\text {alg }}$ be the continuous semi-algebraic function defined by $\varphi(x)=1 /\left(x^{2}+(1-x)^{2}\right)$. As $A$ is real closed, also $\varphi \circ f \in A$. But $(\varphi \circ f) \cdot\left(f^{2}+(1-f)^{2}\right)=1$ as we see by evaluating at each $x \in X$. Since $x^{2}+(1-x)^{2} \geq 1 / 2$ on $R$ we have $f^{2}+(1-f)^{2} \geq 1 / 2$.

(10.4) Observation. Let $\mathfrak{a}$ be an ideal of a real closed ring $A$ and let $f \in A$ be such that $0 \leq f \leq 1 \bmod \mathfrak{p}$ for all $\mathfrak{p} \in V(\mathfrak{a})$. Then $g:=(f \wedge 1) \vee 0$ satisfies $f-g \in \sqrt{\mathfrak{a}}$ and $0 \leq g \leq 1 \bmod \mathfrak{p}$ for all $\mathfrak{p} \in \operatorname{Spec} A$.

Proof. We omit the easy proof. 
For a subset $S$ of an arbitrary ring $A, D(S)$ denotes the set of all prime ideals $\mathfrak{p}$ of $A$ with $\mathfrak{p} \cap S=\emptyset$ (hence $D(S)$ is in general not the complement of $V(S)$ ). The set $D(S)$ is an inverse closed subset of Spec $A$, i.e. $D(S)$ is closed in the inverse topology of $\operatorname{Spec} A$, which has the quasi-compact open subsets of $\operatorname{Spec} A$ as a basis of closed sets. Moreover, if $S$ is multiplicatively closed, then a straightforward calculation shows that the map Spec $\varphi:$ Spec $S^{-1} A \rightarrow \operatorname{Spec} A$ induced by the localization $\varphi: A \rightarrow S^{-1} A$ is a homeomorphism onto $D(S)$ with inverse $\mathfrak{p} \mapsto \mathfrak{p} \cdot\left(S^{-1} \cdot A\right)$. Hence if $A \rightarrow S^{-1} A$ is injective, then $D(S)$ contains all minimal prime ideals of $A$ (as $\operatorname{Spec} \varphi$ is dominant).

(10.5) Theorem. Let $A \subseteq B \subseteq R$ be rings such that $R$ is real closed and $A$ contains the holomorphy ring of $R$. Then $A$ and $B$ are real closed, convex subrings of $R, B=S^{-1} \cdot A$ with $S:=B^{*} \cap A$, and for every ideal $\mathfrak{b}$ of $B$ the map

$$
\iota: V(\mathfrak{b})^{\max } \rightarrow V(\mathfrak{b} \cap A)^{\max }, \quad \mathfrak{n} \mapsto \sqrt[\max ]{\mathfrak{n} \cap A},
$$

is a homeomorphism.

Proof. $A$ is convex in $R$, since for all $f \in R$ and $a \in A$ with $0 \leq f \leq a$ we have $1+a^{2} \in R^{*}$ and $f /\left(1+a^{2}\right) \in \operatorname{Hol}(R)$, so $f=\left(1+a^{2}\right) \cdot f /\left(1+a^{2}\right) \in$ $A \cdot \operatorname{Hol}(R) \subseteq A$. As a convex subring of a real closed ring, $A$ is real closed. Hence, also $B$ is real closed and convex in $R$.

Let $S:=B^{*} \cap A$. Clearly $S$ is a multiplicatively closed set of non-zerodivisors of $A$ containing 1. If $b \in B$, then $s:=1 /\left(1+b^{2}\right) \in \operatorname{Hol}(R) \subseteq A \subseteq B$, hence $s \in B^{*} \cap A$. Since $b=s^{-1} \cdot b /\left(1+b^{2}\right)$ and $b /\left(1+b^{2}\right) \in \operatorname{Hol}(\bar{R}) \subseteq A$ it follows that $B=S^{-1} \cdot A$.

Since $1 /\left(1+f^{2}\right) \in \operatorname{Hol}(R)$ for all $f \in B$ and $\left|f /\left(1+f^{2}\right)\right|, 1 /\left(1+f^{2}\right) \leq 1$ we have $f /\left(1+f^{2}\right), 1 /\left(1+f^{2}\right) \in A$ for all $f \in B$. Consequently, every ideal $\mathfrak{b}$ of $B$ is generated by $\mathfrak{b} \cap A$.

Since Spec $A$ is completely normal we can apply (10.2), and by (10.1) it is enough to show that $f B \cap A$ and $(1-f) B \cap A$ are coprime for all $f \in B$.

Since $B$ is real closed and $\operatorname{Hol}(B) \subseteq A$ we know from (10.3) that $1 /\left(f^{2}+(1-f)^{2}\right) \in A$ for all $f \in B$. Consequently, for every $f \in A$ we have

$$
\frac{f^{2}}{f^{2}+(1-f)^{2}} \in f B \cap A, \quad \frac{(1-f)^{2}}{f^{2}+(1-f)^{2}} \in(1-f) B \cap A
$$

and

$$
1=\frac{f^{2}}{f^{2}+(1-f)^{2}}+\frac{(1-f)^{2}}{f^{2}+(1-f)^{2}},
$$

as required.

Observe that for a proper convex subring $A$ of a real closed ring $B$, there is some $\mathfrak{m} \in(\operatorname{Spec} A)^{\text {max }}$ with $\mathfrak{m} \cdot B=B$. To see this take some $b \in B \backslash A$. 
Then $f:=1+b^{2} \notin A$, since $-f \leq b \leq f$. Since $f \in B^{*}$ and $f^{-1} \in A$, any maximal ideal of $A$ containing $f^{-1}$ satisfies $\mathfrak{m} \cdot B=B$.

Not every inverse closed subset of $\operatorname{Spec} A$ containing $(\operatorname{Spec} A)^{\mathrm{min}}$ is of the form $D(S)$, where $S=B^{*} \cap A$ and $B$ is a real closed overring of $A$ as in (10.5). To see an example, let $X:=\mathbb{R}^{n}$. Then $X$ is cozero complemented (cf. $\left[\right.$ He-Wo]), i.e. $Z:=(\operatorname{Spec} C(X))^{\mathrm{min}}$ is an inverse closed subset of $\operatorname{Spec} C(X)$. On the other hand, if $B$ is a real closed ring containing $A:=C(X)$ as a convex subring, then by (10.5), (Spec $B)^{\max }$ is homeomorphic to $\beta X$. Since $X=\mathbb{R}^{n}, \beta X$ is not boolean, so (Spec $\left.B\right)^{\text {max }}$ is not boolean and $Z$ cannot be the image of $\operatorname{Spec} B \rightarrow \operatorname{Spec} C(X)$. The same argument also works for $C^{*}(X)$ instead of $C(X)$.

\section{The convex closure of a super real closed ring}

(11.1) Lemma. Let $\varphi: A \rightarrow B$ be a surjective ring homomorphism between real closed rings (actually, lattice ordered rings are enough). Let $F \subseteq C \subseteq B$ be such that $F$ is finite and $C$ is countable. Suppose we have a section $\iota: F \rightarrow A$ such that $\iota: F \rightarrow \iota F$ is an order isomorphism. Then $\iota$ can be extended to a section $\iota^{\prime}: C \rightarrow A$ which is an order isomorphism onto $\iota^{\prime}(C)$.

Proof. Straightforward by induction on the cardinality of $F$.

(11.2) Proposition and Definition. Let $A$ be a real closed ring. Let $S:=\{f \in A \mid f \geq 0, f$ a non-zero-divisor and $\forall a \in A: 0 \leq a \leq f \Rightarrow f \mid a\}$. Then

(i) For all $a, b \in A$ and $f \in S$ with $0 \leq a \leq b$ and $f \mid b$ we have $f \mid a$.

(ii) $1 \in S$ and $S \cdot S \subseteq S$.

(iii) The ring $B:=S^{-1} A$ is the largest real closed ring so that $A$ is convex in $B$, more precisely: if $C$ is a real closed overring of $A$ such that $A$ is convex in $C$, then there is a unique A-embedding of $C$ into $B$.

$B$ is called the convex closure of $A$.

(iv) For $f \in A$ we have $f \in B^{*}$ if and only if $|f| \in S$ if and only if $f^{2} \in S$.

(v) For $f \in S$ and $f \leq g \in A$ we have $g \in S$, in particular $g \mid f$.

Proof. (i) Let $0 \leq a \leq b$ and let $b^{\prime} \in A$ with $b=f b^{\prime}$. By replacing $b^{\prime}$ with $\left|b^{\prime}\right|$ if necessary, we may assume that $b^{\prime} \geq 0$. Let $c:=b^{\prime} \vee 1 \in A$. Then $f b^{\prime} \leq f c$ and since $c \geq 1, c$ is a unit in $A$. Hence with $a^{\prime}:=a / c^{2} \in A$ we have $0 \leq a^{\prime} \leq f$. Since $f \in S$ there is some $a^{\prime \prime} \in A$ with $a^{\prime}=f a^{\prime \prime}$. So $a=a^{\prime} c^{2}=f a^{\prime \prime} c^{2}$ and $f \mid a$. 
(ii) Clearly $1 \in S$. Let $f, g \in S$ and $0 \leq a \leq f g, a \in A$. Since $f \in S$, (i) says that there is $a_{1} \in A$ with $a=a_{1} f$. Hence $0 \leq a_{1} f \leq f g$ and since $f$ is a non-zero-divisor, $f \geq 0$ implies $0 \leq a_{1} \leq g$. Since $g \in S$, there is some $a_{2} \in A$ with $a_{1}=a_{2} g$. Thus $a=a_{1} f=a_{2} g f$, i.e. $f g \mid a$. This shows that $f g \in S$, so $S \cdot S \subseteq S$.

Before we prove (iii), (iv) and (v) we need

Claim. Let $C$ be a real closed overring of $A$ such that $A$ is convex in $C$. If $f \in C^{*} \cap A$, then $|f| \in S$.

Proof of the Claim. Since $A \subseteq C, f$ is a non-zero-divisor of $A$, hence so is $|f|$. Take $a \in A$ with $0 \leq a \leq|f|$. Then $0 \leq a /|f| \leq 1$ in $C$, as $f \in C^{*}$. Since $A$ is convex in $C$ we get $|f| \mid a$ in $A$. This shows the claim.

(iii) $A$ is convex in $B=S^{-1} A$, since for $0 \leq a / f \leq a^{\prime}, a, a^{\prime} \in A, f \in S$ we have $0 \leq a \leq a^{\prime} f$, hence by (i), $f \mid a$ in $A$ and $a / f \in A$.

Now let $C$ be a real closed overring of $A$ such that $A$ is convex in $C$. We already know from (10.5) that $C=T^{-1} A$ with $T=C^{*} \cap A$. By the claim we have $T^{\prime}:=\left\{t^{2} \mid t \in T\right\} \subseteq S$. Since $a / t=a t / t^{2}$ for all $a \in A, t \in T$, we have $C=T^{-1} A=T^{-1} A \subseteq S^{-1} A=B$.

(iv) If $f \in B^{*} \cap A$, then $|f| \in S$ by the claim. If $|f| \in S$, then $f^{2}=|f|^{2}$ $\in S$. If $f^{2} \in S$, then $f^{2} \in B^{*}$ and $f \cdot\left(f \cdot\left(f^{2}\right)^{-1}\right)=1$, that is, $f \in B^{*}$.

(v) Let $S \ni f \leq g \in A$. Then $0 \leq f \in B^{*}$ and therefore $f \leq g$ implies $g \in B^{*}$. By (iv), $g=|g| \in S$.

Note that by [Kn-Zh2, Theorem 9.15], the convex closure of a real closed ring is the Prüfer hull (cf. [Kn-Zh1]) of that ring.

Our next goal is to prove that the convex closure of a super real closed ring is again a super real closed ring in a natural way. In order to carry out this task we will prove that the set $S$ from (11.2) is stable under the application of $s \in \Upsilon$.

First a characterization of the elements from $S$ in the case of the super real closed rings $C(X) / \mathfrak{a}$ for a $z$-radical ideal $\mathfrak{a}$ of $C(X)$.

(11.3) Proposition. For $f \in C(X), f \geq 0$, and every $z$-ideal $\mathfrak{a}$ of $C(X)$ the following are equivalent:

(i) $\forall g \in C(X): 0 \leq g \bmod \mathfrak{a} \leq f \bmod \mathfrak{a} \Rightarrow f \bmod \mathfrak{a} \mid g \bmod \mathfrak{a}$.

(ii) $\forall g \in C(X): 0 \leq g \leq f \Rightarrow f \bmod \mathfrak{a} \mid g \bmod \mathfrak{a}$.

(iii) $D:=\{f \neq 0\}$ is $C^{*}$-embedded into $X$ at $\mathfrak{f}:=\{\{a=0\} \mid a \in \mathfrak{a}\}$, i.e. for every $H \in C^{*}(D)$ there are $Z \in \mathfrak{f}$ and an extension $h \in C(X)$ of $\left.H\right|_{Z \cap D}$.

Proof. (iii) $\Rightarrow$ (i). Let $g \in C(X)$ with $0 \leq g \leq f$ modulo a. By (11.1) there are $g^{\prime}, f^{\prime} \in C(X)$ with $g-g^{\prime}, f-f^{\prime} \in \mathfrak{a}$ and $0 \leq g^{\prime} \leq f^{\prime}$. By replacing $f$ by $f^{\prime}$ and $g$ by $g^{\prime}$ we may assume that $0 \leq g \leq f$. Let $H:=\left.g\right|_{D} /\left.f\right|_{D} \in C^{*}(D)$. So by assumption there is $Z \in \mathfrak{f}$ such that $\left.H\right|_{Z \cap D}$ can be extended to an 
$h \in C(X)$. Then $\{h \cdot f-g=0\} \supseteq Z \cap D$. On $Z \backslash D$ we have $0 \leq g \leq f \equiv 0$, hence $\{h \cdot f-g=0\} \supseteq Z$ and this shows that $f \bmod \mathfrak{a} \mid g \bmod \mathfrak{a}$.

(i) $\Rightarrow$ (ii) is a weakening.

(ii) $\Rightarrow$ (iii). Suppose $\forall g \in C(X): 0 \leq g \leq f \Rightarrow f \bmod \mathfrak{a} \mid g \bmod \mathfrak{a}$. Let $H \in C^{*}(D)$. We have to find some $Z \in \mathfrak{f}$ such that $\left.H\right|_{Z \cap D}$ can be extended to a continuous function on $X$. Since $H=H^{+}-H^{-}$we may assume that $H \geq 0$ and of course also that $H \leq 1$. Since $D$ is the cozero set of $f$, $\left.f\right|_{D} \cdot H$ has an extension $g \in C(X)$ with $g(x)=0$ for all $x \in\{f=0\}$. Since $0 \leq H \leq 1$ and $f \geq 0$ we have $0 \leq g \leq f$. By (ii), $f \bmod \mathfrak{a} \mid g \bmod \mathfrak{a}$ and there is some $h \in C(X)$ with $f \cdot h-g \in \mathfrak{a}$. So $Z:=\{f \cdot h-g=0\}$ is a set from $\mathfrak{f}$ such that $h$ extends $\left.H\right|_{Z \cap D}$.

(11.4) Corollary. If $f \in C(X), f \neq 0$, and $D:=\{f \neq 0\}$, then the following are equivalent:

(i) $\forall g \in C(X): 0 \leq g \leq f \Rightarrow f \mid g$.

(ii) $\{f \neq 0\}$ is $C^{*}$-embedded into $X$.

Proof. By (11.3) applied to the $z$-ideal $\mathfrak{a}=\{0\}$.

(11.5) Corollary. Let $A$ be the super real closed ring $C(X) / \mathfrak{a}$ for some $z$-radical ideal $\mathfrak{a}$ of $C(X)$. Let $a \in A, a \geq 0$, and let $s \in \Upsilon$ with $s(t) \geq 0$ $(t \geq 0)$. Then

$$
\forall b \in A: 0 \leq b \leq a \Rightarrow a|b \Leftrightarrow \forall b \in A: 0 \leq b \leq s(a) \Rightarrow s(a)| b .
$$

Proof. Pick $f \in C(X), f \geq 0$, with $a=f \bmod \mathfrak{a}$. By (11.3), $\forall b \in A$ : $0 \leq b \leq a \Rightarrow a \mid b$ if and only if $D:=\{f \neq 0\}$ is $C^{*}$-embedded into $X$ at $\mathfrak{f}:=\{\{h=0\} \mid h \in \mathfrak{a}\}$. This latter property only depends on the cozero set of $f$, hence it holds for $f$ if and only if it holds for $s \circ f$, and this shows the corollary.

(11.6) Proposition. Let $A$ be a real closed ring and let $f \in A$ with $f \geq 0$. Then the following are equivalent:

(i) $\forall a \in A: 0 \leq a \leq f \Rightarrow f \mid a$.

(ii) The natural map between the holomorphy rings $\operatorname{Hol}(A) \rightarrow \operatorname{Hol}\left(A_{f}\right)$ is surjective.

Proof. First note that $\operatorname{Hol}(A) \rightarrow A \rightarrow A_{f}$ has indeed values in $\operatorname{Hol}\left(A_{f}\right)$. To see this let $a \in \operatorname{Hol}(A)$. There is some $N \in \mathbb{N}$ with $|a| \leq_{\beta} N(\beta \in \operatorname{Sper} A$, the real spectrum of $A$ ). But then also $|a / 1| \leq_{\alpha} N$ for all $\alpha \in$ Sper $A_{f}$, hence $a / 1 \in \operatorname{Hol}\left(A_{f}\right)$.

(ii) $\Rightarrow\left(\right.$ i). Let $0 \leq a \leq f$. Then $0 \leq a / f \leq 1$ in $A_{f}$, so by (ii) there is some $g \in A$ such that $g / 1=a / f$ in $A_{f}$. We claim that $g \cdot f=a$ in $A$. To see this take a prime ideal $\mathfrak{p}$ of $A$. If $f \in \mathfrak{p}$, then also $a \in \mathfrak{p}$, as $0 \leq a \leq f$. If $f \notin \mathfrak{p}$, then $g f-a \in \mathfrak{p}$, since $g / 1=a / f$ in $A_{f}$. Hence $g f-a \in \mathfrak{p}$ for all prime ideals $\mathfrak{p}$ of $A$, and this shows $g f=a$ in $A$. 
(i) $\Rightarrow$ (ii). Let $a \in A$ and $N \in \mathbb{N}$ be such that $\left|a / f^{n}\right| \leq_{\alpha} N$ for all $\alpha \in \operatorname{Sper} A_{f}$. Then $0 \leq a^{+} / N f^{n} \leq_{\alpha} 1$ and $0 \leq a^{-} / N f^{n} \leq_{\alpha} 1$ for all $\alpha \in \operatorname{Sper} A_{f}$. So in order to show that $\operatorname{Hol}(A) \rightarrow \operatorname{Hol}\left(A_{f}\right)$ is surjective we may assume that $a \geq 0$ and $0 \leq a / f^{n} \leq_{\alpha} 1$ for all $\alpha \in$ Sper $A_{f}$. This assumption implies $0 \leq f \cdot a \leq f^{n+1}$ in $A$. By (i) there is some $g \in A$ with $f^{n+1} \cdot g=f \cdot a$. But this means that $g$ is a preimage of $a / f^{n}$ in $A$. Now, $g$ need not be in $\operatorname{Hol}(A)$, but we can modify it in order to get this property as well: Let $g^{\prime}:=(g \wedge 1) \vee-1$. Then $\left|g^{\prime}\right| \leq 1$ and for $\mathfrak{p} \in \operatorname{Spec} A$ with $f \notin \mathfrak{p}, 0 \leq f \cdot a \leq f^{n+1}$ and the equation $f^{n+1} \cdot g=f \cdot a$ shows that $f^{n+1} \cdot g^{\prime}=f \cdot a \bmod \mathfrak{p}$.

(11.7) Corollary. Let $B$ be the convex closure of $C(X)$. Then the image of $(\operatorname{Spec} B)^{\max }$ under $\operatorname{Spec} B \rightarrow \operatorname{Spec} C(X)$ is contained in $z$-Spec $C(X)$.

Proof. $B$ is the localization of $C(X)$ at

$$
S:=\{f \in C(X) \mid f \geq 0, f \text { a non-zero-divisor and }
$$

$$
\forall g \in C(X): 0 \leq g \leq f \Rightarrow f \mid g\}
$$

by (11.2). If $\mathfrak{p} \in \operatorname{Spec} C(X)$ with $\mathfrak{p} \cap S=\emptyset$, then also $\sqrt[z]{\mathfrak{p}} \cap S=\emptyset$, since membership in $S$ only depends on the cozero set of a function by (11.4). Since the image of $(\operatorname{Spec} B)^{\max }$ under $\operatorname{Spec} B \rightarrow \operatorname{Spec} C(X)$ is the set $D(S)^{\max }$, this proves the corollary.

(11.8) Lemma. If $A \subseteq B$ are real closed rings, then

$$
\operatorname{Hol}(A)=A \cap \operatorname{Hol}(B) .
$$

Proof. $B$ is isomorphic to a ring of functions $X \rightarrow R$ for some set $X$ and some real closed field $R$. For such a ring, the assertion is obvious.

(11.9) Lemma. Let $A$ be a super real closed ring and let $f, a \in A$ and $r, s \in \Upsilon$ be such that $|a / s(f)| \leq 1$ in $A_{s(f)}$. Then there is some $g \in A$ with $|g| \leq|r(f)|$ such that $g=r(f) \cdot(a / s(f))$ in $A_{s(f)}$.

Proof. First we reduce to the case where $A$ is generated by $f$ and $a$ as a super real closed ring. Let $B$ be the $\Upsilon$-subring of $A$ generated by $f$ and $a$. Since $|a / s(f)| \leq 1$ in $A_{s(f)}$ we also have $|a / s(f)| \leq 1$ in $B_{s(f)}$ (cf. (11.8)). If we find an element $g \in B$ with $|g| \leq|r(f)|$ such that $g=r(f) \cdot(a / s(f))$ in $B_{s(f)}$, then also $g=r(f) \cdot(a / s(f))$ in $A_{s(f)}$. This argument shows that we may replace $A$ by $B$, hence we may assume that $A$ is generated by $f$ and $a$ as a super real closed ring.

Let $\varphi: C\left(\mathbb{R}^{2}\right) \rightarrow A$ be the unique super homomorphism which sends $x$ to $f$ and $y$ to $a$. Since $s(f) / 1=\varphi(s(x)) / 1$ is a unit in $A_{s(f)}$ we get a natural map $\psi: C\left(\mathbb{R}^{2}\right)_{s(x)} \rightarrow A_{s(f)}$, which is surjective, as $\varphi$ is surjective. Since 
$|a / s(f)| \leq 1$ in $A_{s(f)}$, there is some $F(x, y) \in C\left(\mathbb{R}^{2}\right)$ such that

$$
\psi\left(\frac{F(x, y)}{s(x)}\right)=\frac{a}{s(f)} \text { and } \quad\left|\frac{F(x, y)}{s(x)}\right| \leq 1 \quad \text { in } C\left(\mathbb{R}^{2}\right)_{s(x)}
$$

(cf. (11.1)). Let $D:=\left\{(u, v) \in \mathbb{R}^{2} \mid s(u) \neq 0\right\}$. Then $\left.F\right|_{D} /\left.s\right|_{D} \in C(D)$ is of absolute value less than 1 . Therefore the function

$$
G(u, v):= \begin{cases}r(u) \cdot \frac{\left.F\right|_{D}}{\left.s\right|_{D}}(u, v) & \text { if } u \neq 0 \\ 0 & \text { if } u=0\end{cases}
$$

is continuous on $\mathbb{R}^{2},|G(u, v)| \leq|r(u)|$ for all $u, v \in \mathbb{R}$ and $G \cdot s=r \cdot F$ on $\mathbb{R}^{2}$. Thus $G / 1=r(x) \cdot(F / s)$ in $C\left(\mathbb{R}^{2}\right)_{s(x)}$.

We take $g:=\varphi(G) \in A$. Then $|g| \leq|r(f)|$ and $g=r(f) \cdot(a / s(f))$ in $A_{s(f)}$.

(11.10) Proposition. Let $A$ be a super real closed ring, let $f \in A$ and let $r, s \in \Upsilon$. Then the natural map $A_{s(f)} \rightarrow A_{r(f) \cdot s(f)}$ induces an isomorphism of the holomorphy rings

$$
\operatorname{Hol}\left(A_{s(f)}\right) \rightarrow \operatorname{Hol}\left(A_{r(f) \cdot s(f)}\right)
$$

Hence $\operatorname{Hol}\left(A_{s(f)}\right)$ is independent of $s$ and equal to $\operatorname{Hol}\left(A_{f}\right)$.

Proof. First we show that the natural map $A_{s(f)} \rightarrow A_{r(f) \cdot s(f)}$ is injective. Let $a \in A$ be such that $a / s(f)^{n}=0$ in $A_{r(f)} \cdot s(f)$. Then $(r(f) \cdot s(f))^{k} \cdot a$ $=0$ for some $k$ and we will show that $s(f) \cdot a=0$. This proves $a / s(f)^{n}=0$ in $A_{s(f)}$ as desired. Let $\mathfrak{p}$ be a minimal prime ideal of $A$. If $a \in \mathfrak{p}$, then $s(f) \cdot a \in \mathfrak{p}$. If $a \notin \mathfrak{p}$, then $(r(f) \cdot s(f))^{k} \cdot a=0$ implies $r(f) \in \mathfrak{p}$ or $s(f) \in \mathfrak{p}$. Since $\mathfrak{p}$ is minimal, it is $\Upsilon$-radical, hence $f \in \mathfrak{p}$ in any case and so $s(f) \in \mathfrak{p}$ if $a \notin \mathfrak{p}$.

This shows that $s(f) \cdot a \in \mathfrak{p}$ for all $\mathfrak{p} \in \operatorname{Spec} A$, hence $s(f) \cdot a=0$.

It remains to show that $\operatorname{Hol}\left(A_{s(f)}\right) \rightarrow \operatorname{Hol}\left(A_{r(f)} \cdot s(f)\right)$ is surjective. Pick $a \in A$ such that $a /(r(f) \cdot s(f))^{n} \in \operatorname{Hol}\left(A_{r(f)} \cdot s(f)\right)$. Clearly we may assume that $\left|a /(r(f) \cdot s(f))^{n}\right| \leq 1$ in $A_{r(f)} \cdot s(f)$. Now we apply (11.9) to $s$ and $(r \cdot s)^{n}$ (instead of $r, s)$. We get some $g \in A$ with $|g| \leq|s(f)|$ such that

$$
g=s(f) \cdot \frac{a}{(r(f) \cdot s(f))^{n}} \quad \text { in } A_{r(f) \cdot s(f)} .
$$

It follows that $g / s(f)$ is a preimage of $a /(r(f) \cdot s(f))^{n}$ in $\operatorname{Hol}\left(A_{s(f)}\right)$ under $\operatorname{Hol}\left(A_{s(f)}\right) \rightarrow \operatorname{Hol}\left(A_{r(f)} \cdot s(f)\right)$.

Remark. By (11.6), Proposition (11.10) says that $A_{s(f)}$ is a convex subring of $A_{r(f) \cdot s(f)}$ (note that at the beginning of the proof of Proposition (11.10) it is shown that $r(f) / 1$ is a non-zero-divisor in $\left.A_{s(f)}\right)$.

(11.11) Corollary. Let $A$ be a super real closed ring, let $f \in A, f \geq 0$, and let $s \in \Upsilon$ with $s(u) \geq 0$ for $u \geq 0$. Then with $S:=\{f \in A \mid f \geq 0$ 
and $\forall a \in A: 0 \leq a \leq f \Rightarrow f \mid a\}$ we have $f \in S \Leftrightarrow s(f) \in S$. In particular $D(S)^{\max } \subseteq \Upsilon$-Spec $A$.

Proof. By (11.6), $f \in S$ if and only if $\operatorname{Hol}(A) \rightarrow \operatorname{Hol}\left(A_{f}\right)$ is an isomorphism. Since $\operatorname{Hol}\left(A_{f}\right) \rightarrow \operatorname{Hol}\left(A_{f} \cdot s(f)\right)$ and $\operatorname{Hol}\left(A_{s(f)}\right) \rightarrow \operatorname{Hol}\left(A_{f} \cdot s(f)\right)$ are isomorphisms by (11.10), we get $f \in S \Leftrightarrow s(f) \in S$.

(11.12) Theorem. Let $A$ be a super real closed ring and let $B$ be the convex closure of $A$. Then there is a unique expansion of $B$ to a super real closed ring having $A$ as a super real closed subring.

Proof. By (11.2), $B=S^{-1} A$ with $S:=\{f \in A \mid f \geq 0, f$ a non-zerodivisor and $\forall a \in A: 0 \leq a \leq f \Rightarrow f \mid a\}$. By (11.11), $S$ is $\Upsilon$-multiplicatively closed and by (7.4) the theorem follows from $B=S^{-1} A$.

\section{Real closures over proconstructible subsets of $\Upsilon$-Spec $A$}

(12.1) Proposition. Let $I$ be an index set and let $M_{i}$ be a super real closed field for $i \in I$. Let $A$ be a super real closed subring of $R:=\prod_{i \in I} M_{i}$. For every semi-algebraic function $f: \mathbb{R}_{\text {alg }}^{k} \rightarrow \mathbb{R}_{\text {alg }}$ and every $a \in R^{k}$ let $f^{R}(a):=\left(f^{M_{i}}\left(a_{i}\right)\right)_{i \in I} \in R$. Then

$$
D:=\left\{f^{R}(a) \mid k \in \mathbb{N}, f: \mathbb{R}_{\mathrm{alg}}^{k} \rightarrow \mathbb{R}_{\mathrm{alg}} \text { semi-algebraic, } a \in A^{k}\right\}
$$

is again a super real closed subring of $R$ and this $\mathscr{L}_{\Upsilon}$-structure is the only one which expands the pure ring $D$ to a super real closed ring having $A$ as a super real closed subring.

Proof. Let $F \in C\left(\mathbb{R}^{n}\right)$ and let $f_{1}, \ldots, f_{n}: \mathbb{R}_{\text {alg }}^{k} \rightarrow \mathbb{R}_{\text {alg }}$ be semi-algebraic. Let $a \in R^{k}$. We have to show that $F_{R}\left(f_{1}^{R}(a), \ldots, f_{n}^{R}(a)\right) \in D$. By (7.2), there are $t \in \Upsilon$ and $G \in C\left(\mathbb{R}^{n} \times \mathbb{R}\right)$ with

$$
F\left(x_{1}, \ldots, x_{n}\right) \cdot t(y)=G\left(x_{1} \cdot y, \ldots, x_{n} \cdot y, y\right) \quad\left((\bar{x}, y) \in \mathbb{R}^{n} \times \mathbb{R}\right) .
$$

Recall that every semi-algebraic function $\mathbb{R}_{\text {alg }}^{k} \rightarrow \mathbb{R}_{\mathrm{alg}}$ is of the form

$$
\sum_{i=1}^{l} \frac{g_{j}}{h_{j}} \cdot \chi_{j},
$$

where $\chi_{j}$ are semi-algebraic characteristic functions with $\mathbb{R}_{\text {alg }}^{k}=\bigcup_{j}\left\{\chi_{j}=1\right\}$, $g_{j}, h_{j}$ are continuous semi-algebraic functions $\mathbb{R}_{\text {alg }}^{k} \rightarrow \mathbb{R}_{\text {alg }}$ and $h_{j}$ has no zeros on $\left\{\chi_{j} \neq 0\right\}$ (by convention $\left(g_{j} / h_{j}\right) \cdot \chi_{j}(x)=0$ if $\left.h_{j}(x)=0\right)$.

Pick such functions $\chi_{\mu j}, g_{\mu j}, h_{\mu j}$ for each $f_{\mu}$. By selecting a common refinement of the supports of the $\chi_{\mu j}$, we may assume that the $\chi_{\mu j}$ do not depend on $\mu \in\{1, \ldots, n\}$, so we write $\chi_{j}$ instead of $\chi_{\mu j}$. Moreover, by suitably changing the $g_{\mu j}$ 's we may also assume that the $h_{\mu j}$ do not depend on $\mu \in\{1, \ldots, n\}$, so we write $h_{j}$ instead of $h_{\mu j}$. 
Let $f: \mathbb{R}_{\mathrm{alg}}^{2 l+k} \rightarrow \mathbb{R}_{\mathrm{alg}}$ be semi-algebraic, defined by $f\left(x_{1}, y_{1}, \ldots, x_{l}, y_{l}, \bar{z}\right)$

$$
:= \begin{cases}\frac{x_{j}}{y_{j}} \cdot \chi_{j}(\bar{z}) & \text { if } y_{j} \cdot \chi_{j}(\bar{z}) \neq 0 \text { for some } j \in\{1, \ldots, l\}, \\ 0 & \text { if } y_{j} \cdot \chi_{j}(\bar{z})=0 \text { for all } j \in\{1, \ldots, l\} .\end{cases}
$$

Observe that $f$ is well defined, since $\mathbb{R}_{\text {alg }}^{k}=\bigcup_{j}\left\{\chi_{j}=1\right\}$. It is enough to show that

$$
F_{R}\left(f_{1}^{R}(a), \ldots, f_{n}^{R}(a)\right)=f^{R}\left(c_{1}, d_{1}, \ldots, c_{l}, d_{l}, a\right) \in D
$$

with $c_{j}:=G_{R}\left(g_{1 j}^{R}(a), \ldots, g_{n j}^{R}(a), h_{j}^{R}(a)\right) \in A, d_{j}=t^{R}\left(h_{j}(a)\right) \in A(1 \leq j \leq l)$.

We prove $(*)$ coordinatewise. Let $i \in I$. There is a unique $j$ with $\chi_{j}^{M_{i}}\left(a_{i}\right)$ $=1$. Then for each $\mu \in\{1, \ldots, n\}$ we have $f_{\mu}^{R}(a)_{i}=f_{\mu}^{M_{i}}\left(a_{i}\right)=g_{\mu j}^{M_{i}}\left(a_{i}\right) / h_{j}^{M_{i}}\left(a_{i}\right)$, hence

$$
\begin{aligned}
F_{R}\left(f_{1}^{R}(a), \ldots, f_{n}^{R}(a)\right)_{i} & =F_{M_{i}}\left(f_{1}^{M_{i}}\left(a_{i}\right), \ldots, f_{n}^{M_{i}}\left(a_{i}\right)\right) \\
& =F_{M_{i}}\left(\frac{g_{1 j}^{M_{i}}\left(a_{i}\right)}{h_{j}^{M_{i}}\left(a_{i}\right)}, \ldots, \frac{g_{n j}^{M_{i}}\left(a_{i}\right)}{h_{j}^{M_{i}}\left(a_{i}\right)}\right) .
\end{aligned}
$$

By (7.4) we know that

$$
\begin{aligned}
F_{M_{i}}\left(\frac{g_{1 j}^{M_{i}}\left(a_{i}\right)}{h_{j}^{M_{i}}\left(a_{i}\right)}, \ldots, \frac{g_{n j}^{M_{i}}\left(a_{i}\right)}{h_{j}^{M_{i}}\left(a_{i}\right)}\right) & \cdot t^{M_{i}}\left(h_{j}^{M_{i}}\left(a_{i}\right)\right) \\
& \left.=G_{M_{i}}\left(g_{1 j}^{M_{i}}\left(a_{i}\right), \ldots, g_{n j}^{M_{i}}\left(a_{i}\right)\right), h_{j}^{M_{i}}\left(a_{i}\right)\right),
\end{aligned}
$$

in other words

$$
F_{R}\left(f_{1}^{R}(a), \ldots, f_{n}^{R}(a)\right)_{i} \cdot d_{j i}=c_{j i}
$$

where $d_{j i}, c_{j i}$ denote the $i$ th components of $d_{j}, c_{j}$ respectively. By the choice of the $h_{j}$ and since $\chi_{j}^{M_{i}}\left(a_{i}\right)=1$, we know that $d_{j i} \neq 0$. Thus

$$
F_{R}\left(f_{1}^{R}(a), \ldots, f_{n}^{R}(a)\right)_{i}=\frac{c_{j i}}{d_{j i}},
$$

which is the $i$ th component of $f^{R}\left(c_{1}, d_{1}, \ldots, c_{l}, d_{l}, a\right)$, since the pure field $M_{i}$ is an elementary extension of $\mathbb{R}_{\text {alg }}$.

Hence $D$ is a super real closed subring of $R$. Now let $E$ be another super real closed ring expanding $D$ such that $A$ is a super real closed subring of $E$. We must show that $F_{D}=F_{E}$ for every $F \in C\left(\mathbb{R}^{n}\right), n \in \mathbb{N}$. Pick $i \in I$. Let $\pi: D \rightarrow M_{i}$ be the projection and let $M$ be the image of $\pi$. Then $M$ is the quotient field of the image of $A \rightarrow D \rightarrow M_{i}$, hence the kernel of $\pi$ is a maximal ideal, thus an $\Upsilon$-radical ideal with respect to every

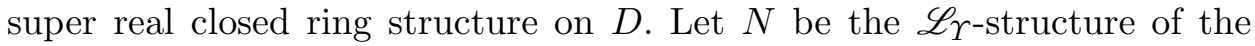
pure field $M$ imposed by $E$ according to (6.3). Then $\pi: D \rightarrow N$ is a super homomorphism. Composing $\pi$ with the inclusion $A \rightarrow D$ (which is a super 
homomorphism by assumption) shows that the natural map $A \rightarrow N$ is a super homomorphism as well. By (6.3) and (7.4) we must have $N=M$. Thus $\pi: E \rightarrow M_{i}$ is a super homomorphism, in other words we have

$$
F_{E}\left(f_{1}^{R}(a), \ldots, f_{n}^{R}(a)\right)_{i}=F_{M_{i}}\left(f_{1}^{M_{i}}\left(a_{i}\right), \ldots, f_{n}^{M_{i}}\left(a_{i}\right)\right)
$$

for all $F, f_{1}, \ldots, f_{n}, a$ as in $(\dagger)$ above. Now the computation following ( $\dagger$ ) and the fact that $A$ is a super real closed subring of $D$ force $F_{D}=F_{E}$.

We have two consequences of (12.1).

(12.2) Corollary. Let $A$ be a super real closed ring and let $E(A)$ be the epimorphic hull of $A$ (cf. [St, Definition 8.3]) in the category of commutative, unital rings. Then there is a unique expansion of $E(A)$ to a super real closed ring such that the canonical homomorphism $A \rightarrow E(A)$ is a super homomorphism.

Proof. Let $Z$ be the closure of (Spec $A)^{\mathrm{min}}$ in the constructible topology. Then $E(A)$ is the ring $B$ of constructible sections of $A$ above $Z$ (the definition of this ring can be found in [Schw1, Chapter I, Section 2]). This follows easily from the characterization of epimorphisms [Schw-Ma, Theorem 5.2], and basic results from [St]. By the characterization of the elements of $B$ in [Schw1], $B$ is the ring $D$ defined for $A \subseteq \prod_{\mathfrak{p} \in Z} \mathrm{qf}(A / \mathfrak{p})$ in (12.1). Hence the corollary is an instance of (12.1).

Note that by [Schw2, Theorem 1.2] the epimorphic hull of every real closed ring in the category of commutative rings is real closed.

The second consequence of (12.1) concerns rings of abstract semi-algebraic functions over proconstructible subsets of $\Upsilon$-Spec $A$ as defined in [Schw1, Chapter I, Section 2]. We first recall some tools from [Schw1].

(12.3) Reminder. Let $A$ be a ring. Let $\alpha, \beta \in$ Sper $A$ (the real spectrum of $A$ ) and assume that $\beta$ is a specialization of $\alpha$. Then $\operatorname{supp} \beta / \operatorname{supp} \alpha$ is a convex prime ideal in $A / \operatorname{supp} \alpha$ and there is a largest convex valuation ring $C$ of $k(\alpha)$ (the ordered residue field of the support of $\alpha$ ) such that $\mathfrak{m} \cap A / \operatorname{supp} \alpha=\operatorname{supp} \beta / \operatorname{supp} \alpha$, where $\mathfrak{m}$ is the maximal ideal of $C$. The set $\mathfrak{m}$ can be defined as

$$
\mathfrak{m}=\left\{y \in k(\alpha) \mid y^{n} \in \text { the convex hull of } \operatorname{supp} \beta / \operatorname{supp} \alpha\right\}
$$

and then

$$
C=\{c \in k(\alpha) \mid c \cdot \mathfrak{m} \subseteq \mathfrak{m}\} .
$$

We write $C_{\alpha \beta}$ for this valuation ring, $\mathfrak{m}_{\alpha \beta}$ for its maximal ideal and $\kappa_{\alpha \beta}$ for the residue field of $C_{\alpha \beta}$. Observe that the map $A / \operatorname{supp} \beta \rightarrow \kappa_{\alpha \beta}$ factors through an embedding $k(\beta) \rightarrow \kappa_{\alpha \beta}$; we write $\lambda_{\alpha \beta}$ for this map.

We will apply this construction to real closed rings exclusively. In this case orderings of $A$ are identified with their support, hence we write $C_{\mathfrak{p q}}$, 
$\mathfrak{m}_{\mathfrak{p q}}, \ldots$ instead of $C_{\alpha \beta}, \mathfrak{m}_{\alpha \beta}, \ldots$ where $\mathfrak{p}=\operatorname{supp} \alpha$ and $\mathfrak{q}=\operatorname{supp} \beta$. Also observe that in this case $k(\mathfrak{p})$ is real closed and

$$
\mathfrak{m}_{\mathfrak{p q}}=\text { the convex hull of } \mathfrak{q} / \mathfrak{p} \text {. }
$$

(12.4) LEMmA. If $A$ is a super real closed ring and $\mathfrak{p}, \mathfrak{q} \in \Upsilon$-Spec $A$ with $\mathfrak{p} \subseteq \mathfrak{q}$, then $C_{\mathfrak{p q}}$ is a super real closed subring of $k(\mathfrak{p})$ with $\Upsilon$-radical maximal ideal and the map $\lambda_{\mathfrak{p q}}$ is a super homomorphism.

Proof. This follows from (9.2) applied to the super real closed rings $A / \mathfrak{p} \subseteq k(\mathfrak{p})$ and the $\Upsilon$-radical ideal $\mathfrak{q} / \mathfrak{p}$ of $A / \mathfrak{p}$.

Recall that for a real closed ring $A$ and a proconstructible subset $P$ of Spec $A$ the real closure of $A$ above $P$ is the following ring: Let $\Phi: A \rightarrow$ $\prod_{\mathfrak{p} \in P} k(\mathfrak{p})$ be the evaluation map and let $A_{0}$ be the image of $\Phi$. Let $D$ be the ring defined for $A_{0}$ according to (12.1). Then the real closure of $A$ above $P$ is the subring of all elements $\left(s_{\mathfrak{p}}\right)_{\mathfrak{p} \in P} \in D$ which are compatible, i.e. for all $\mathfrak{p}, \mathfrak{q} \in P$ with $\mathfrak{p} \subseteq \mathfrak{q}$ we have

$$
s_{\mathfrak{p}} \bmod (\mathfrak{q} / \mathfrak{p})=\lambda_{\mathfrak{p q}}\left(s_{\mathfrak{q}}\right) .
$$

(12.5) TheOREM. Let $A$ be a super real closed ring and let $P \subseteq \Upsilon$-Spec $A$ be proconstructible. Let $B$ be the real closure of $A$ above $P$. Then there is a unique expansion of $B$ to a super real closed ring such that the natural map $A \rightarrow B$ is a super homomorphism.

Proof. Let $\Phi: A \rightarrow \prod_{\mathfrak{p} \in P} k(\mathfrak{p})$ be the evaluation map and let $A_{0}$ be the image of $\Phi$. Since $P \subseteq \Upsilon$-Spec $A$ we know that $\Phi$ is a super homomorphism and $A_{0}$ is a super real closed subring of $\prod_{\mathfrak{p} \in P} k(\mathfrak{p})$. Let $D$ be the ring defined for $A_{0}$ according to (12.1). We claim that $B$ is a super real closed subring of $D$. Hence we must show that, for every $F \in C\left(\mathbb{R}^{n}\right)$, all $s_{1}:=\left(s_{1 \mathfrak{p}}\right)_{\mathfrak{p} \in P}, \ldots, s_{n}:=\left(s_{n \mathfrak{p}}\right)_{\mathfrak{p} \in P} \in B$ and each specialization $\mathfrak{p} \subseteq \mathfrak{q}$ inside $P$ we have

$$
F_{k(\mathfrak{p})}\left(s_{1 \mathfrak{p}}, \ldots, s_{n \mathfrak{p}}\right) \bmod (\mathfrak{q} / \mathfrak{p})=\lambda_{\mathfrak{p q}}\left(F_{k(\mathfrak{q})}\left(s_{1 \mathfrak{q}}, \ldots, s_{n \mathfrak{q}}\right)\right) .
$$

Since $\mathfrak{q} / \mathfrak{p}$ is an $\Upsilon$-radical ideal of $A / \mathfrak{p}$ we have

$$
F_{k(\mathfrak{p})}\left(s_{1 \mathfrak{p}}, \ldots, s_{n \mathfrak{p}}\right) \bmod (\mathfrak{q} / \mathfrak{p})=F_{A / \mathfrak{q}}\left(s_{1 \mathfrak{p}} \bmod (\mathfrak{q} / \mathfrak{p}), \ldots, s_{n \mathfrak{p}} \bmod (\mathfrak{q} / \mathfrak{p})\right)
$$

Since $\lambda_{\mathfrak{p q}}$ is a super homomorphism we know that

$$
\lambda_{\mathfrak{p q}}\left(F_{k(\mathfrak{q})}\left(s_{1 \mathfrak{q}}, \ldots, s_{n \mathfrak{q}}\right)\right)=F_{\kappa_{\mathfrak{p q}}}\left(\lambda_{\mathfrak{p q}}\left(s_{1 \mathfrak{q}}\right), \ldots, \lambda_{\mathfrak{p q}}\left(s_{n \mathfrak{q}}\right)\right) .
$$

Finally, since $A / \mathfrak{q}$ is a super real closed subring of $\kappa_{\mathfrak{p q}}$ and $s_{i \mathfrak{p}} \bmod (\mathfrak{q} / \mathfrak{p})=$ $\lambda_{\mathfrak{p q}}\left(s_{i \mathfrak{q}}\right)$ we see that $(*)$ indeed holds.

Hence $B$ can be expanded to a super real closed ring such that the natural homomorphism $A \rightarrow B$ is a super homomorphism. The uniqueness statement follows from the uniqueness statement in (12.1), since $D$ is also the ring obtained from $B$ according to (12.1). 


\section{The complete ring of quotients of a super real closed ring.} Recall that a subset of a ring $A$ is called dense if it is not annihilated by nonzero elements of $A$. For a ring extension $A \subseteq B$ and any element $b \in B$ we define $b^{-1} \cdot A:=\{a \in A \mid a \cdot b \in A\}$. Clearly $b^{-1} \cdot A$ is an ideal of $A$. Recall from [FGL, Section 1.4] that $B$ is called a rational extension (the name "fractional extension" is also used in the literature) of $A$ if $b^{-1} \cdot A$ is a dense subset of $B$ for all $b \in B$.

By [FGL, 1.9], every commutative ring has a largest rational extension $Q(A)$, called the complete ring of quotients, which is uniquely determined up to $A$-isomorphism. In this section we prove that for every super real closed ring $A$ there is a unique expansion of $Q(A)$ to a super real closed ring having $A$ as a super real closed subring (see (13.5)).

(13.1) Lemma. Let $A$ be a super real closed ring and let $B$ be an overring of $A$. Let $b, b_{1}, \ldots, b_{n} \in B$ be such that $b$ does not annihilate $\left(b_{1}^{-1} \cdot A\right) \cap \cdots \cap$ $\left(b_{n}^{-1} \cdot A\right)$. Then for every $F \in C\left(\mathbb{R}^{n}\right)$ there are $a, a^{*} \in A$ with $a \cdot b \neq 0$ such that whenever $B$ is expanded to a super real closed ring having $A$ as a super real closed subring, we have

$$
a \cdot F_{B}\left(b_{1}, \ldots, b_{n}\right)=a^{*} .
$$

Proof. By assumption there is some $a^{\prime} \in A$ with $a^{\prime} \cdot b \neq 0$ such that $a^{\prime} \cdot b_{i} \in A$ for each $i$.

By (7.2) there are $G \in C\left(\mathbb{R}^{n} \times \mathbb{R}\right)$ and $t \in \Upsilon$ with $F\left(x_{1}, \ldots, x_{n}\right) \cdot t(y)=$ $G\left(x_{1} \cdot y, \ldots, x_{n} \cdot y, y\right)$ for all $\left(x_{1}, \ldots, x_{n}, y\right) \in \mathbb{R}^{n} \times \mathbb{R}$. Now we take $a:=$ $t_{A}\left(a^{\prime}\right)$ and $a^{*}:=G_{A}\left(a^{\prime} \cdot b_{1}, \ldots, a^{\prime} \cdot b_{n}, a^{\prime}\right) \in A$.

First we show $a \cdot b=t_{A}\left(a^{\prime}\right) \cdot b \neq 0$. Since $a^{\prime} \cdot b \neq 0$ there is a minimal prime ideal $\mathfrak{p}$ of $B$ with $a^{\prime} \cdot b \notin \mathfrak{p}$. Since $\mathfrak{p}$ is minimal, $\mathfrak{p}$ is $\Upsilon$-radical. Since $a^{\prime} \notin \mathfrak{p}$, also $t_{A}\left(a^{\prime}\right) \notin \mathfrak{p}$. As $b \notin \mathfrak{p}$ we get $t_{A}\left(a^{\prime}\right) \cdot b \notin \mathfrak{p}$ as desired.

Finally, we prove $(*)$ for every expansion of $B$ to a super real closed ring having $A$ as a super real closed subring: We have $a \cdot F_{B}\left(b_{1}, \ldots, b_{n}\right)=$ $t_{A}\left(a^{\prime}\right) \cdot F_{B}\left(b_{1}, \ldots, b_{n}\right)=t_{B}\left(a^{\prime}\right) \cdot F_{B}\left(b_{1}, \ldots, b_{n}\right)=G_{B}\left(b_{1} \cdot a^{\prime}, \ldots, b_{n} \cdot a^{\prime}, a^{\prime}\right)=$ $G_{A}\left(b_{1} \cdot a^{\prime}, \ldots, b_{n} \cdot a^{\prime}, a^{\prime}\right)=a^{*}$.

(13.2) Proposition. Let $A$ be a super real closed subring of the super real closed ring $B$. Let $D$ be the set of all $d \in B$ for which $d^{-1} \cdot A$ is a dense subset of $B$. Then $D$ is a super real closed subring of $B$ and the induced $\mathscr{L}_{\Upsilon}$-structure on $D$ is the unique one for which $D$ is a super real closed ring having $A$ as a super real closed subring.

Proof. First we show that $D$ is a super real closed subring of $B$. Let $F \in C\left(\mathbb{R}^{n}\right)$ and let $b_{1}, \ldots, b_{n} \in D$. We must show that the ideal $F_{B}\left(b_{1}, \ldots, b_{n}\right)^{-1} \cdot A$ of $A$ is a dense subset of $B$. Pick $b \in B, b \neq 0$. Since each $b_{i}^{-1} \cdot A$ is a dense subset of $B$, also $\left(b_{1}^{-1} \cdot A\right) \cap \cdots \cap\left(b_{n}^{-1} \cdot A\right)$ is a dense subset of $B$. As $b \neq 0, b$ is not in the annihilator of $\left(b_{1}^{-1} \cdot A\right) \cap \cdots \cap\left(b_{n}^{-1} \cdot A\right)$. Pick $a, a^{*}$ 
according to $b, b_{1}, \ldots, b_{n}$ and $F$ as in (13.1). Then $(*)$ of (13.1) shows $a \in$ $F_{B}\left(b_{1}, \ldots, b_{n}\right)^{-1} \cdot A$. Since $a \cdot b \neq 0, b$ does not annihilate $F_{B}\left(b_{1}, \ldots, b_{n}\right)^{-1} \cdot A$, as desired.

Hence $D$ is a super real closed subring of $B$. Let $E$ be another expansion of the pure ring $D$ to a super real closed ring having $A$ as a super real closed subring. Let $b_{1}, \ldots, b_{n} \in D$. We must show $F_{D}\left(b_{1}, \ldots, b_{n}\right)=F_{E}\left(b_{1}, \ldots, b_{n}\right)$. Otherwise $b:=F_{D}\left(b_{1}, \ldots, b_{n}\right)-F_{E}\left(b_{1}, \ldots, b_{n}\right) \neq 0$. Since all $b_{i}$ are in $D, b$ is not in the annihilator of $\left(b_{1}^{-1} \cdot A\right) \cap \cdots \cap\left(b_{n}^{-1} \cdot A\right)$. Pick $a, a^{*}$ as in (13.1) with respect to the ring $D$, the elements $b, b_{1}, \ldots, b_{n} \in D$ and the function $F$. By (13.1) we have $a \cdot b \neq 0$ and $a \cdot F_{D}\left(b_{1}, \ldots, b_{n}\right)=a^{*}=a \cdot F_{E}\left(b_{1}, \ldots, b_{n}\right)$, which contradicts the definition of $b$.

(13.3) Reminder. Let $A$ be a ring and let $\mathscr{O}_{\operatorname{Spec} A}$ be the sheaf of the affine scheme of $A$. Hence the stalk of $\mathscr{O}_{\operatorname{Spec} A}$ at $\mathfrak{p}$ is the localization $A_{\mathfrak{p}}$ of $A$ at $\mathfrak{p}$ and for every $U \subseteq \operatorname{Spec} A$ open,

$$
\begin{aligned}
& \mathscr{O}_{\text {Spec } A}(U)=\left\{f=\left(f_{\mathfrak{p}}\right)_{\mathfrak{p} \in U}\right. \in \prod_{\mathfrak{p} \in U} A_{\mathfrak{p}} \mid \text { for all } \mathfrak{p} \in U \text { there are } a, s \in A \\
&\text { with } \left.\mathfrak{p} \in D(s) \subseteq U \text { and } f_{\mathfrak{q}}=\frac{a}{s} \text { in } A_{\mathfrak{q}}(\mathfrak{q} \in D(s))\right\} .
\end{aligned}
$$

Fix an open subset $U \subseteq \operatorname{Spec} A$ and let $B:=\mathscr{O}_{\text {Spec } A}(U)$. We shall consider $B$ as an $A$-module via the natural homomorphism $\varepsilon: A \rightarrow B$. If $\mathfrak{p} \in U$, then the natural map $A_{\mathfrak{p}} \rightarrow B_{\mathfrak{p}}$ between the localizations of the $A$-modules $A$ and $B$ is easily seen to be injective. If $A$ is reduced, then this map is an isomorphism, since the map $B_{\mathfrak{p}} \rightarrow A_{\mathfrak{p}}$ induced by the projection $B \rightarrow A_{\mathfrak{p}}$ is injective.

(13.4) Proposition. Let $A$ be a super real closed ring. If $U \subseteq \operatorname{Spec} A$ is open and $U \subseteq \Upsilon$-Spec $A$, then there is a unique $\Upsilon$-structure on $B:=$ $\mathscr{O}_{\text {Spec } A}(U)$ such that $B$ is super real closed and the canonical map $A \rightarrow B$ is a super homomorphism. Moreover $B$ is a super real closed subring of the super real closed ring $C:=\prod_{\mathfrak{p} \in U} A_{\mathfrak{p}}$.

Proof. Pick $f_{1}, \ldots, f_{n} \in B$ and $F \in C\left(\mathbb{R}^{n}\right)$. We have to show that $F_{C}\left(f_{1}, \ldots, f_{n}\right) \in B$. Pick $\mathfrak{p} \in U$. Then there are $a_{i}, s \in A$ with $\mathfrak{p} \in D(s) \subseteq U$ such that $\left(f_{i}\right)_{\mathfrak{q}}=a_{i} / s$ in $A_{\mathfrak{q}}$ for all $\mathfrak{q} \in D(s)$. By (7.2) there are $G \in$ $C\left(\mathbb{R}^{n} \times \mathbb{R}\right)$ and $t \in \Upsilon$ with $F\left(x_{1}, \ldots, x_{n}\right) \cdot t(y)=G\left(x_{1} \cdot y, \ldots, x_{n} \cdot y, y\right)$ for all $\left(x_{1}, \ldots, x_{n}, y\right) \in \mathbb{R}^{n} \times \mathbb{R}$. Since $U \subseteq \Upsilon$-Spec $A$, every $A_{\mathfrak{q}}$ with $\mathfrak{q} \in D(s)$ is super real and $t_{A}(s) \notin \mathfrak{q}$ for all $\mathfrak{q} \in D(s)$. Thus we may apply (7.4) to get

$$
F_{A_{\mathfrak{q}}}\left(\frac{a_{1}}{s}, \ldots, \frac{a_{n}}{s}\right)=\frac{G_{A}\left(a_{1}, \ldots, a_{n}, s\right)}{t_{A}(s)} \quad(\mathfrak{q} \in D(s)) .
$$

This proves $F_{C}\left(f_{1}, \ldots, f_{n}\right) \in B$. We equip $B$ with this $\mathscr{L}_{\Upsilon \text {-structure and }}$ denote the resulting super real closed ring by $\mathscr{B}$. 
It remains to show the uniqueness statement. Fix a super structure on $B$ which turns $B$ into a super real closed ring $\mathscr{D}$ such that the natural map $\varepsilon: A \rightarrow \mathscr{D}$ is a super homomorphism. Then $\varepsilon(A \backslash \mathfrak{p})$ is a $\Upsilon$-multiplicatively closed subset of $\mathscr{D}$, hence $\mathscr{D} \rightarrow \mathscr{D}_{\mathfrak{p}}=\varepsilon(A \backslash \mathfrak{p})^{-1} \cdot \mathscr{D}$ is a super homomorphism. Hence the composite map $A \rightarrow \mathscr{D}_{\mathfrak{p}}$ is also a super homomorphism. By (13.3) the induced map $A_{\mathfrak{p}} \rightarrow B_{\mathfrak{p}}$ is an isomorphism. Since there is only one superstructure on $A_{\mathfrak{p}}$ such that $A \rightarrow A_{\mathfrak{p}}$ is a super homomorphism we get $\mathscr{D}_{\mathfrak{p}}=\mathscr{B}_{\mathfrak{p}}=A_{\mathfrak{p}}$. Since $\mathscr{D}$ and $\mathscr{B}$ are super real closed subrings of $C$, also $\mathscr{D}=\mathscr{B}$ as super real closed rings.

(13.5) TheOrem. Let $A$ be a super real closed ring and let $Q$ be the complete ring of quotients as defined in [FGL, Section 1]. Then there is a unique $\mathscr{L}_{Y}$-structure on $Q$ such that $Q$ is a super real closed ring having $A$ as a super real closed subring.

Proof. Uniqueness follows from (13.2).

By [St, Satz 11.3], $Q$ contains the epimorphic hull $E$ of $A$. As explained in [FGL, Section 1.4], $Q$ is also the complete ring of quotients of $E$. Hence by (12.2), we may replace $A$ with $E$. In particular, we may assume that $A$ is a super real closed ring which is von Neumann regular.

It follows that $\operatorname{Spec} A=\Upsilon$-Spec $A$ and by (13.4), we have a unique way to expand the rings $\mathscr{O}_{\mathrm{Spec} A}(U)(U \subseteq \operatorname{Spec} A$ open $)$ to super real closed rings; moreover for all $V \subseteq U \subseteq \operatorname{Spec} A$ open, the restriction map $\mathscr{O}_{\text {Spec } A}(U) \rightarrow$ $\mathscr{O}_{\text {Spec } A}(V)$ preserves the $\mathscr{L}_{\Upsilon}$-structure. Hence the filtered family of all the $\mathscr{O}_{\text {Spec } A}(U)$ with $U \subseteq \operatorname{Spec} A$ open and dense, together with the restriction maps, is a filtered family in the category of super real closed rings with super real homomorphisms. Since this category has direct limits, we may define the super real closed ring

$$
H(A):=\underline{\lim }\left\{\mathscr{O}_{\operatorname{Spec} A}(U) \mid U \subseteq \operatorname{Spec} A \text { open and dense }\right\} .
$$

We can easily see that the underlying ring of $H(A)$ is also the direct limit of the $\mathscr{O}_{\text {Spec } A}(U)(U \subseteq$ Spec $A$ open and dense) in the category of commutative rings. But, as $A$ is reduced, this ring is $A$-isomorphic to $Q$. This last fact is folklore, we sketch a proof:

For each $b \in Q$ let $U_{b}:=\operatorname{Spec} A \backslash V\left(b^{-1} A\right)$. Since $b^{-1} A$ is dense in $A, U_{b}$ is dense in $\operatorname{Spec} A$ and we may define an element $\theta(b) \in H(A)$ as the image of the section

$$
\mathfrak{p} \mapsto \frac{b s}{s} \in A_{\mathfrak{p}} \quad \text { for some } s \in b^{-1} A \backslash \mathfrak{p}
$$

of $\mathscr{O}_{\operatorname{Spec} A}\left(U_{b}\right)$ under the natural map $\mathscr{O}_{\operatorname{Spec} A}\left(U_{b}\right) \rightarrow H(A)$. It is a routine matter to check that $\theta: Q \rightarrow H(A)$ defines an $A$-algebra homomorphism. Since $A$ is reduced and each $b^{-1} A$ is dense in $B(b \in Q)$, it turns out that $\theta$ is injective. Hence we may identify $Q$ with a subring of $H(A)$. 
On the other hand, $H(A)$ is a rational extension of $A$, since each $\mathscr{O}_{\text {Spec } A}(U)$ with $U \subseteq \operatorname{Spec} A$ open and dense is a rational extension of $A$ (as follows from (13.3) with the help of the isomorphisms $A_{\mathfrak{p}} \rightarrow B_{\mathfrak{p}}, \mathfrak{p} \in U$ ).

14. Entering varieties of $\Upsilon$-Spec $A$. Here we prove a theorem $((14.5))$ about the location of $V(\mathfrak{a})$ with respect to $V\left(\mathfrak{a}^{\Upsilon}\right)$ if $\mathfrak{a}$ is a finitely generated ideal of a super real closed ring $A$. By the general theory of real closed rings, every point $\mathfrak{q}$ of Spec $A$ outside $V(\mathfrak{a})$ that specializes to some point in $V(\mathfrak{a})$ has to enter $V(\mathfrak{a})$ at a first point, namely at $\mathfrak{q}+\sqrt{\mathfrak{a}}$. Theorem (14.5) says that this entrance point is minimal in $V(\mathfrak{a})$. This is in strong contrast to the semi-algebraic case (we assume some basic knowledge of real geometry for this argument, cf. [BCR]): Let $A$ be the ring of continuous semi-algebraic functions $\mathbb{R}^{n} \rightarrow \mathbb{R}$ and let $f \in A$ be the distance function to the closed unit ball in $\mathbb{R}^{n}$. Then for each prime ideal $\mathfrak{q}$ of $A$ outside $V(f)$ that specializes to a point in $V(f)$, the point $\mathfrak{q}+\sqrt{(f)}$ lies in $\widetilde{S^{n-1}}=V(g)$, where $g$ is the distance function to the sphere $S^{n-1}$. This point is not minimal in $V(f)$ !

(14.1) Lemma. Let $A$ be a super real closed ring and let $\mathfrak{p}$ be a prime ideal of $A$. If $f \in A$ and $\sqrt{\mathfrak{p}+f \cdot A}$ is a proper $\Upsilon$-radical ideal, then $f \in \mathfrak{p}$.

Proof. First we show

$$
\sqrt{\mathfrak{p}+f \cdot A}=\left\{\left.g \in A|| g\right|^{n} \leq|f| \bmod \mathfrak{p} \text { for some } n \in \mathbb{N}\right\} .
$$

The inclusion $\supseteq$ follows from the convexity condition for the real closed ring $A / \mathfrak{p}$. Conversely, since $1 \notin \mathfrak{p}+f \cdot A$ we have $|f \cdot h|<1 \bmod \mathfrak{p}$ for all $h \in A$. Therefore $f^{2} h^{2}<|f| \bmod \mathfrak{p}$ for all $h \in A$ and it is straightforward to prove that the set on the right hand side of $(*)$ is an ideal of $A$. This ideal is radical and contains $\mathfrak{p}$ and $f$. This shows $(*)$.

Now take a barrier function $L: \mathbb{R} \rightarrow \mathbb{R}($ see $[\operatorname{Tr} 1,(7.1)])$. Since $\sqrt{\mathfrak{p}+f \cdot A}$ is $\Upsilon$-radical we have $L^{A}(f) \in \sqrt{\mathfrak{p}+f \cdot A}$. Let $A_{0}$ be a finitely generated super real closed subring of $A$ containing $f$ such that $L^{A}(f) \in \sqrt{\mathfrak{p} \cap A_{0}+f \cdot A_{0}}$. By (*) applied to $A_{0}$ and $\mathfrak{p} \cap A_{0}$, there is some $n \in \mathbb{N}$ such that $\left|L^{A}(f)^{n}\right| \leq$ $|f| \bmod \mathfrak{p} \cap A_{0}$. Let $k \in \mathbb{N}$ be such that $A_{0}$ is of the form $C\left(\mathbb{R}^{k}\right) / I$ for some $\Upsilon$-radical ideal $I$ of $C\left(\mathbb{R}^{k}\right)$ and let $\mathfrak{q} \in \operatorname{Spec} C\left(\mathbb{R}^{k}\right)$ be such that $\mathfrak{q} / I=\mathfrak{p} \cap A_{0}$. Take a preimage $F$ of $f$ in $C\left(\mathbb{R}^{k}\right)$. Then $\left|L^{A}(f)^{n}\right| \leq|f| \bmod \mathfrak{p} \cap A_{0}$ means $\left|(L \circ F)^{n}\right| \leq|f| \bmod \mathfrak{q}$. By $[\operatorname{Tr} 1,(7.2)]$ we get $F \in \mathfrak{q}$. This in turn implies $f \in \mathfrak{p}$.

(14.2) Conollary. Let $A$ be a super real closed ring, let $\mathfrak{a} \subseteq A$ be an ideal and let $f_{1}, \ldots, f_{n} \in A$. If there is an $\Upsilon$-radical prime ideal with $\mathfrak{a} \subseteq \mathfrak{p} \subsetneq \mathfrak{p}+\left(f_{1}, \ldots, f_{n}\right) \subsetneq A$, then $\sqrt{\mathfrak{a}+\left(f_{1}, \ldots, f_{n}\right)}$ is not $\Upsilon$-radical.

Observe that in the case $A=C(X), \mathfrak{a}$ might not be the largest $z$-radical ideal contained in $\mathfrak{a}+\sqrt{\left(f_{1}, \ldots, f_{n}\right)}$, even if $\mathfrak{a}=\mathfrak{p}$ is $z$-radical: take $z$-radical prime ideals $\mathfrak{p} \subsetneq \mathfrak{p}_{1} \subsetneq \mathfrak{p}_{2}, \mathfrak{a}:=\mathfrak{p}$ and $f \in \mathfrak{p}_{2} \backslash \mathfrak{p}_{1}$. 
Proof. Suppose $\sqrt{\mathfrak{a}+\left(f_{1}, \ldots, f_{n}\right)}$ is $\Upsilon$-radical. Then

$$
\begin{aligned}
\sqrt[r]{\mathfrak{p}+\sqrt{\left(f_{1}, \ldots, f_{n}\right)}} & =\sqrt[r]{\mathfrak{p}+\mathfrak{a}+\sqrt{\left(f_{1}, \ldots, f_{n}\right)}} \\
& =\sqrt[r]{\mathfrak{p}}+\sqrt[r]{\mathfrak{a}+\sqrt{\left(f_{1}, \ldots, f_{n}\right)}} \\
& =\mathfrak{p}+\sqrt[r]{\sqrt{\mathfrak{a}+\left(f_{1}, \ldots, f_{n}\right)}}=\mathfrak{p}+\sqrt{\mathfrak{a}+\left(f_{1}, \ldots, f_{n}\right)} \\
& =\mathfrak{p}+\sqrt{\mathfrak{a}}+\sqrt{\left(f_{1}, \ldots, f_{n}\right)}=\mathfrak{p}+\sqrt{\left(f_{1}, \ldots, f_{n}\right)},
\end{aligned}
$$

hence $\mathfrak{p}+\sqrt{\left(f_{1}, \ldots, f_{n}\right)}=\sqrt{\mathfrak{p}+\left(f_{1}^{2}+\cdots+f_{n}^{2}\right)}$ is $\Upsilon$-radical and by (14.1) we get $f_{1}^{2}+\cdots+f_{n}^{2} \in \mathfrak{p}$, thus $f_{1}, \ldots, f_{n} \in \mathfrak{p}$.

(14.3) Lemma. If $\mathfrak{a}$ is an ideal of a super real closed ring $A$ and $\mathfrak{q}$ is an $\Upsilon$-radical prime ideal of $A$, then either $\mathfrak{q}+\sqrt{\mathfrak{a}} \in V(\mathfrak{a})^{\min }$, or $\mathfrak{q}+\sqrt{\mathfrak{a}}$ is $\Upsilon$-radical.

Proof. We may assume that $\mathfrak{q}+\sqrt{\mathfrak{a}}$ is a proper ideal, hence $\mathfrak{q}+\sqrt{\mathfrak{a}} \in V(\mathfrak{a})$. Take $\mathfrak{p} \in V(\mathfrak{a})^{\text {min }}$ with $\mathfrak{a} \subseteq \mathfrak{p} \subseteq \mathfrak{q}+\sqrt{\mathfrak{a}}$. Then $\mathfrak{p}^{\Upsilon} \subseteq \mathfrak{p},(\mathfrak{q}+\sqrt{\mathfrak{a}})^{\Upsilon}$, so $\mathfrak{p}$ and $(\mathfrak{q}+\sqrt{\mathfrak{a}})^{\Upsilon}$ are comparable. If $(\mathfrak{q}+\sqrt{\mathfrak{a}})^{\Upsilon} \subseteq \mathfrak{p}$, then, as $\mathfrak{q}$ is $\Upsilon$-radical, $\mathfrak{q} \subseteq(\mathfrak{q}+\sqrt{\mathfrak{a}})^{\Upsilon} \subseteq \mathfrak{p}$, so $\mathfrak{q}+\sqrt{\mathfrak{a}} \subseteq \mathfrak{p}$, which shows that $\mathfrak{q}+\sqrt{\mathfrak{a}}=\mathfrak{p} \in V(\mathfrak{a})^{\text {min }}$.

Now assume that $\mathfrak{p} \subsetneq(\mathfrak{q}+\sqrt{\mathfrak{a}})^{r}$. Then $\sqrt{\mathfrak{a}} \subseteq \mathfrak{p} \subseteq(\mathfrak{q}+\sqrt{\mathfrak{a}})^{r}$. Since $\mathfrak{q}$ is $\Upsilon$-radical, also $\mathfrak{q} \subseteq(\mathfrak{q}+\sqrt{\mathfrak{a}})^{\Upsilon}$, hence $\mathfrak{q}+\sqrt{\mathfrak{a}}=(\mathfrak{q}+\sqrt{\mathfrak{a}})^{\Upsilon}$ is $\Upsilon$-radical.

(14.4) Remark. If $A=C(X)$ and $\mathfrak{q}$ is a $z$-radical prime ideal of $C(X)$, then the proof of $(14.3)$, where " $\Upsilon$-radical" is replaced by " $z$-radical" and the ideal construction $I^{\Upsilon}$ is replaced by the diamond construction $I^{\diamond}$ (cf. $[\operatorname{Tr} 1,(3.4)])$, shows that either $\mathfrak{q}+\sqrt{\mathfrak{a}} \in V(\mathfrak{a})^{\min }$, or $\mathfrak{q}+\sqrt{\mathfrak{a}}$ is $z$-radical.

(14.5) Theorem. Let $\mathfrak{a}$ be an ideal of a super real closed ring $A$ and let $\mathfrak{q}$ be an $\Upsilon$-radical prime ideal of $A$, not containing $\mathfrak{a}$ and such that $1 \notin \mathfrak{a}+\mathfrak{q}$. If $\mathfrak{q}+\sqrt{\mathfrak{a}}=\mathfrak{q}+\sqrt{\left(f_{1}, \ldots, f_{n}\right)}$ for some $f_{1}, \ldots, f_{n}$, then $\mathfrak{q}+\sqrt{\mathfrak{a}} \in V(\mathfrak{a})^{\min }$.

Proof. By (14.2), $\mathfrak{q}+\sqrt{\mathfrak{a}}$ is not $\Upsilon$-radical; then apply (14.3).

Recall from [Tr1, (4.5)] the tubular ideal $O(\mathfrak{a})$ attached to an ideal $\mathfrak{a}$ of a ring $A$ with normal spectrum: $O(\mathfrak{a})$ is the intersection of all prime ideals $\mathfrak{p}$ of $A$ with $1 \notin \mathfrak{a}+\mathfrak{p}$; we have $O(\mathfrak{a}) \subseteq \mathfrak{a}$ and the set $V(O(\mathfrak{a}))$ of prime ideals of Spec $A$ containing $O(\mathfrak{a})$ is the set $\mathscr{O}(V(\mathfrak{a}))$ of all $\mathfrak{q} \in \operatorname{Spec} A$ specializing to some $\mathfrak{p} \in V(\mathfrak{a})$. All this is explained at the beginning of Section 4 in [Tr1].

(14.6) Corollary. Let $\mathfrak{a}$ be an ideal of a super real closed ring A. If $\mathfrak{b}$ is an ideal of $A$ with $O(\mathfrak{a}) \subseteq \mathfrak{b} \subseteq \mathfrak{a}$ such that $\sqrt{\mathfrak{a}}$ is finitely generated as a radical ideal over $\mathfrak{b}$, then the map

$$
V(\mathfrak{b}) \rightarrow V(\mathfrak{a}), \quad \mathfrak{q} \mapsto \mathfrak{q}+\sqrt{\mathfrak{a}},
$$

maps minimal elements of $V(\mathfrak{b})$ to minimal elements of $V(\mathfrak{a})$. 
Proof. Let $\mathfrak{q} \in V(\mathfrak{b})^{\min }$. If $\mathfrak{q} \in V(\mathfrak{a})$, then $\mathfrak{q}+\sqrt{\mathfrak{a}}=\mathfrak{q}$, and as $\mathfrak{b} \subseteq \mathfrak{a}, \mathfrak{q}$ is already minimal in $V(\mathfrak{a})$. So assume $\mathfrak{q} \in V(\mathfrak{b})^{\text {min }} \backslash V(\mathfrak{a})$. Since $O(\mathfrak{a}) \subseteq \mathfrak{b}$ we have $\mathfrak{q} \in \mathscr{O}(V(\mathfrak{a})) \backslash V(\mathfrak{a})$. By assumption $\sqrt{\mathfrak{a}}=\sqrt{\mathfrak{b}+\left(f_{1}, \ldots, f_{n}\right)}$ for some $f_{1}, \ldots, f_{n} \in C(X)$, hence

$$
\begin{aligned}
\mathfrak{q}+\sqrt{\mathfrak{a}} & =\mathfrak{q}+\sqrt{\mathfrak{b}+\left(f_{1}, \ldots, f_{n}\right)}=\mathfrak{q}+\sqrt{\mathfrak{b}}+\sqrt{\left(f_{1}, \ldots, f_{n}\right)} \\
& =\mathfrak{q}+\sqrt{\mathfrak{b}+\left(f_{1}, \ldots, f_{n}\right)},
\end{aligned}
$$

since $\mathfrak{b} \subseteq \mathfrak{q}$. Thus we can apply (14.5) and get $\mathfrak{q}+\sqrt{\mathfrak{a}} \in V(\mathfrak{a})^{\min }$.

In contrast to (14.6), the map $V(\mathfrak{a}) \rightarrow V(\sqrt[r]{\mathfrak{a}}), \mathfrak{p} \mapsto \sqrt[r]{\mathfrak{p}}$, does not map minimal elements of $V(\mathfrak{a})$ to minimal elements of $V(\sqrt[r]{\mathfrak{a}})$, even if $\mathfrak{a}$ is a principal ideal and $X$ is compact. Here is an example:

ExAmple. Let $I:=[-1,1] \subseteq \mathbb{R}$, let $f \in C(I)$ be the function $f(x)=$ $x \vee 0$, and let $\mathfrak{a}:=f \cdot C(I)$. Let $h \in C(I)$ be defined by $h(x)=0$ if $x \leq 0$ and $h(x)=(\log (x / e))^{-1}$ if $x>0$. Then $x \cdot h(x)=f(x) \cdot h(x) \in \mathfrak{a}$ but no power of $x$ and no power of $h(x)$ is divisible by $f$ in $C(I)$.

This shows that $x \bmod \sqrt{\mathfrak{a}}$ is a zero divisor of $C(I) / \sqrt{\mathfrak{a}}$, thus there is a prime ideal $\mathfrak{p}$ of $C(I)$ minimal over $\mathfrak{a}$, containing $x$. It follows that

$$
\sqrt[r]{\mathfrak{p}}=\sqrt[z]{\mathfrak{p}}=\{g \in C(I) \mid g(0)=0\},
$$

which is not minimal over $\sqrt[z]{\mathfrak{a}}$ : take $\mathfrak{q} \in \operatorname{Spec} C(I)$, $z$-radical, such that the prime filter $\mathfrak{f}$ of closed subsets of $I$ corresponding to $\mathfrak{q}$ contains $[-1,0]$, avoids $\{0\}$ and specializes to 0 . Then $\sqrt[z]{\mathfrak{a}} \subseteq \mathfrak{q} \subsetneq \sqrt[z]{\mathfrak{p}}$.

Acknowledgements. I wish to thank Niels Schwartz for many valuable discussions on real closed rings, and the anonymous referee for significant help in improving an earlier version of the text.

\section{References}

[BCR] J. Bochnak, M. Coste and M.-F. Roy, Real Algebraic Geometry, Ergeb. Math. Grenzgeb. 36, Springer, 1998.

[Che] G. Cherlin, Rings of continuous functions: decision problems, in: Model Theory of Algebra and Arithmetic (Karpacz, 1979), Lecture Notes in Math. 834, Springer, Berlin, 1980, 44-91.

[Da-Wo] H. G. Dales and W. H. Woodin, Super-Real Fields, London Math. Soc. Monogr. 14, Oxford Univ. Press, 1996.

[vdD] L. van den Dries, Tame Topology and o-Minimal Structures, London Math. Soc. Lecture Note Ser. 248, Cambridge Univ. Press, 1998.

[FGL] N. J. Fine, L. Gillman and J. Lambek, Rings of Quotients of Rings of Functions, McGill Univ. Press, Montreal, 1966.

[Gil-Jer] L. Gillman and M. Jerison, Rings of Continuous Functions, Grad. Texts in Math. 43, Springer, 1960. 
[He-Wo] M. Henriksen and R. G. Woods, Cozero complemented spaces; when the space of minimal prime ideals of a $C(X)$ is compact, Topology Appl. 141 (2004), $147-170$.

[Hoc] M. Hochster, Prime ideal structure in commutative rings, Trans. Amer. Math. Soc. 142 (1969), 43-60.

[Ho] W. Hodges, Model Theory, Encyclopedia Math. Appl. 42, Cambridge Univ. Press, 1993.

[Kn-Sch] M. Knebusch and C. Scheiderer, Einführung in die reelle Algebra, Vieweg, 1989.

[Kn-Zh1] M. Knebusch and D. Zhang, Manis Valuations and Prüfer Extensions. I. A New Chapter in Commutative Algebra, Lecture Notes in Math. 1791, Springer, Berlin, 2002.

[Kn-Zh2] - - - Convexity, valuations and Prüfer extensions in real algebra, Doc. Math. 10 (2005), 1-109.

[PS] A. Prestel and N. Schwartz, Model theory of real closed rings, in: Valuation Theory and its Applications, Vol. I (Saskatoon, SK, 1999), Fields Inst. Commun. 32, Amer. Math. Soc., Providence, RI, 2002, 261-290.

[Schw1] N. Schwartz, The basic theory of real closed spaces, Mem. Amer. Math. Soc. 397 (1989).

[Schw2] - Epimorphic extensions and Prüfer extensions of partially ordered rings, Manuscripta Math. 102 (2000), 347-381.

[Schw-Ma] N. Schwartz and J. J. Madden, Semi-Algebraic Function Rings and Reflectors of Partially Ordered Rings, Lecture Notes in Math. 1712, Springer, 1999.

[St] H. H. Storrer, Epimorphismen von kommutativen Ringen, Comment. Math. Helv. 43 (1968), 378-401.

[Tr1] M. Tressl, Computation of the z-radical in $C(X)$, Adv. Geom. 6 (2006), 139175.

[Tr2] - Real closed rings of class $C^{k}$, in preparation.

Universität Passau, IM

Innstr. 33

D-94032 Passau, Germany

E-mail: marcus.tressl@mathematik.uni-regensburg.de

Received 13 January 2006;

in revised form 21 February 2007 